\title{
DIFFEOLOGY OF THE INFINITE HOPF FIBRATION
}

\author{
PATRICK IGLESIAS-ZEMMOUR \\ Einstein Institute, The Hebrew University of Jerusalem \\ Campus Givat Ram, Jerusalem 91904, Israel \\ E-mail: piz@math.huji.ac.il
}

\begin{abstract}
We introduce diffeological real or complex vector spaces. We define the fine diffeology on any vector space. We equip the vector space $\mathcal{H}$ of square summable sequences with the fine diffeology. We show that the unit sphere $\mathcal{S}$ of $\mathcal{H}$, equipped with the subset diffeology, is an embedded diffeological submanifold modeled on $\mathcal{H}$. We show that the projective space $\mathcal{P}$, equipped with the quotient diffeology of $\mathcal{S}$ by $\mathrm{S}^{1}$, is also a diffeological manifold modeled on $\mathcal{H}$. We define the Fubini-Study symplectic form on $\mathcal{P}$. We compute the momentum map of the unitary group $\mathbf{U}(\mathcal{H})$ on the sphere $\mathcal{S}$ and on $\mathcal{P}$. And we show that this momentum map identifies the projective space $\mathcal{P}$ with a diffeological coadjoint orbit of the group $\mathbf{U}(\mathcal{H})$, where $\mathbf{U}(\mathcal{H})$ is equipped with the functional diffeology. We discuss some other properties of the symplectic structure of $\mathcal{P}$. In particular, we show that the image of $\mathcal{P}$ under the momentum map of the maximal torus $\mathbf{T}(\mathcal{H})$ of $\mathbf{U}(\mathcal{H})$ is a convex subset of the space of moments of $\mathbf{T}(\mathcal{H})$, infinitely generated.
\end{abstract}

\section{INTRODUCTION}

Diffeology is a theory that enlarges the scope of differential geometry. It was introduced by J.-M. Souriau [Sou81], and in a slightly different way by K.-T. Chen [Che77] few years before. Diffeology has been successfully tested on «singular» objects like irrational tori [DI85] [Igl85, Igl86] [IL90] or more recently on orbifolds [IKZ05]. This paper shows, in the case of the infinite Hopf fibration, how it can be used in a particular infinite dimensional context. Writing down this example I want to show how everything follows simply from a minimal set of conventions: the unique choice of a diffeology on the standard Hilbert space. No extra structure is needed, and the diffeological framework just works. What needs to be emphasized, in contrast with topological methods, is the very simple formal and coherent use of differential calculus offered by the general diffeological framework. For example, diffeological notions of forms, exterior derivative etc. lead, among other things, to an elegant definition of the momentum map. This seems advantageous to me in this

2000 Mathematics Subject Classification: Primary 58B99.

The paper is in final form and no version of it will be published elsewhere. 
fuzzy world of infinite dimensional spaces, where it is not always clear how differentiability coexists with topology.

Much of the diffeological material presented here is well known by specialists, but some parts are new, required by the subject. In particular, the introduction of diffeological vector spaces, their fine diffeology, and the definition of diffeological manifolds.

The set-theoretic construction of the infinite Hopf fibration is well known, but let us recall it. We consider the Hilbert space $\mathcal{H}$ of square summable complex sequences with the standard hermitian product. The quotient of $\mathcal{H}-\{0\}$ by the multiplicative action of $\mathbf{C}-\{0\}$ is the projective space $\mathcal{P}$ of complex lines of $\mathcal{H}$. This space is equivalent to the quotient of the unit sphere $\mathcal{S} \subset \mathcal{H}$ by the subgroup $S^{1} \subset \mathbf{C}-\{0\}$ of complex numbers of modulus 1. The projection $\mathcal{S} \longrightarrow \mathcal{P}$ is the infinite Hopf fibration. As a topological space, the sphere $\mathcal{S}$ is contractible [Kak43], from which it follows that $\mathcal{S}$ is the $\mathrm{S}^{1}$ topological classifying total space $\mathrm{E}\left(\mathrm{S}^{1}\right)$ and $\mathcal{P}$ its base $\mathrm{B}\left(\mathrm{S}^{1}\right)$.

Now, we consider this construction from the diffeological point of view. After a review of the main diffeological definitions and constructions, we introduce the notion of diffeological vector spaces and some related constructions. The Hilbert space $\mathcal{H}$ is then equipped with the standard hermitian product and with the fine diffeology of vector space. The unit sphere $\mathcal{S}$ of $\mathcal{H}$ inherits the subset diffeology, and the projective space $\mathcal{P}$ is equipped with the quotient diffeology of $\mathcal{S}$ by $\mathrm{S}^{1}$. We shall see that:

a) The sphere $\mathcal{S}$, as well as the projective space $\mathcal{P}$, are diffeological manifolds, both modeled on $\mathcal{H}$.

b) The sphere $\mathcal{S}$ is contractible as a diffeological space.

c) The projection $\mathcal{S} \longrightarrow \mathcal{P}$ is a diffeological bundle, locally trivial. And, the (diffeological) homotopy groups of $\mathcal{P}$ are $\pi_{2}(\mathcal{P})=\mathbf{Z}, \pi_{k}(\mathcal{P})=0$ if $k \neq 2$.

Then we define a certain differential 1-form on $\mathcal{S}$ - an $\mathrm{S}^{1}$-connection form - called Liouville's form, whose curvature generalizes to $\mathcal{P}$ the so-called Fubini-Study symplectic form.

On the other hand, the group $\mathbf{U}(\mathcal{H})$ of unitary transformations of $\mathcal{H}$ acts naturally on $\mathcal{S}$ and $\mathcal{P}$, preserving both the connection form and its curvature. We give a characterization of the functional diffeology of the group $\mathbf{U}(\mathcal{H})$, for $\mathcal{H}$ equipped with the fine diffeology.

Further, we define the space $\mathcal{G}^{*}$ of moments of a diffeological group $\mathrm{G}$ as the set of all left-invariant 1-forms of G. And, because we don't need more generality here, we give the expression of the momentum map, relative to a closed 2-form, in the very particular case where this form is exact and has an invariant primitive. The general definition of the momentum map, for the diffeological context, is given and studied in [PIZ05] (see also [Ig195]). This diffeological momentum map extends Souriau's original definition [Sou70] given in the context of ordinary differential geometry. Then, we apply these constructions to our case, and we see that the characteristic curves of the momentum map $\mu$ of $\mathbf{U}(\mathcal{H})$ on $\mathcal{S}$ are the fibers of the projection $\mathcal{S} \rightarrow \mathcal{P}$. Hence, the momentum map $\mu$ factorizes through $\mathcal{P}$ in the momentum map $m$ of $\mathbf{U}(\mathcal{H})$ on $\mathcal{P}$, more precisely:

d) The momentum map $m$ of $\mathbf{U}(\mathcal{H})$ on $\mathcal{P}$ is injective and identifies the projective space $\mathcal{P}$ with a coadjoint orbit of $\mathbf{U}(\mathcal{H})$, where $\mathbf{U}(\mathcal{H})$ is equipped with the functional diffeology associated to the fine diffeology of $\mathcal{H}$. 
Finally, we introduce the maximal torus $\mathbf{T}(\mathcal{H})$ of the group $\mathbf{U}(\mathcal{H})$ and, after restriction of the previous construction to $\mathbf{T}(\mathcal{H})$, we show that:

e) The image of the momentum map of $\mathbf{T}(\mathcal{H})$ is an infinitely generated convex domain of the vector space $\mathcal{T}^{*}$ of moments of $\mathbf{T}(\mathcal{H})$.

Note that the topological properties of infinite dimensional topological manifolds have been studied more generally than the particular case of the infinite sphere, see for example [Kui65] [Pal65, Pal66]. It would be interesting to have a diffeological parallel for these cases too. It would also be very interesting to classify the coadjoint orbits of $\mathbf{U}(\mathcal{H})$ in this diffeological framework. We shall certainly get all the flag spaces of finite rank, which are direct extensions of the rank one flag case studied here. But it is unclear if they exhaust the whole set of coadjoint orbits.

Thanks. I am pleased to thank, warmly, François Ziegler for all his comments and suggestions which helped me to improve this text. It is a pleasure also to thank the organizers of the conference of Będlewo, Jan Kubarski, Robert Wolak and Jean Pradines who invited me to take part in it. I would not forget to thank also the Hebrew University of Jerusalem for its great hospitality and its warm atmosphere.

\section{REVIEW ON DIFFEOLOGY}

This chapter is a review of the main diffeological constructions used in this article. No proofs are given. The reader can find them in a web document maintained at [PIZ05] or in [Igl85].

\section{Definitions}

1.1. Domains and parametrizations. An n-numerical domain is any open subset of the vector space $\mathbf{R}^{n}, n \geq 0$. A numerical domain is any domain for any $n \in \mathbf{N}$.

A parametrization of a set $\mathrm{X}$ is any map $\mathrm{P}: \mathrm{U} \rightarrow \mathrm{X}$ such that $\mathrm{U}$ is a numerical domain. If $\mathrm{U}$ is an $n$-numerical domain we say that $\mathrm{P}$ is an $n$-parametrization.

1. The set of all the parametrizations of $\mathrm{X}$ defined on $\mathrm{U}$ is denoted by $\operatorname{Param}(\mathrm{U}, \mathrm{X})$.

2. The set of all $n$-parametrizations of $\mathrm{X}$ is denoted by $\operatorname{Param}_{n}(\mathrm{X})$.

3. The set of all parametrizations of $\mathrm{X}$ is denoted by $\operatorname{Param}(\mathrm{X})$.

4. If $\mathrm{P}$ is an $n$-parametrization, we say that the dimension of $\mathrm{P}$ is $n$, we denote it by:

$$
\text { For all } \mathrm{P} \in \operatorname{Param}(\mathrm{X}), \quad \operatorname{dim}(\mathrm{P})=n \quad \Leftrightarrow \quad \mathrm{P} \in \operatorname{Param}_{n}(\mathrm{X}) \text {. }
$$

Let $\mathrm{X}$ be a set, and $x$ be any point of $\mathrm{X}$. A superset of $x$ is any part $\mathrm{V}$ of $\mathrm{X}$ containing $x$. If $\mathrm{X}$ is a topological space, an open superset of $x$ is just a superset of $x$ which is open for the given topology.

1.2. Diffeology and diffeological spaces. A diffeology of a set $\mathrm{X}$ is a subset $\mathcal{D}$ of parametrizations of $\mathrm{X}$, whose elements are called plots, such that the following axioms hold: 
D1. Covering. Any constant parametrization is a plot: for any point $x$ of $\mathrm{X}$ and for any integer $n$, the constant map $\boldsymbol{x}: \mathbf{R}^{n} \longrightarrow \mathrm{X}$, defined by $\boldsymbol{x}(r)=x$ for all $r$ in $\mathbf{R}^{n}$, is a plot.

D2. Locality. For any parametrization $\mathrm{P}: \mathrm{U} \rightarrow \mathrm{X}$, if $\mathrm{P}$ is locally a plot at each point of $\mathrm{U}$ then $\mathrm{P}$ is a plot. This means that, if for every $r$ in $\mathrm{U}$ there exists a superset $\mathrm{V}$ of $r$ such that the restriction $\mathrm{P} \uparrow \mathrm{V}$ is a plot, then $\mathrm{P}$ is a plot.

D3. Smooth compatibility. The composition of a plot with any smooth parametrization of its source is a plot: let $\mathrm{P}: \mathrm{U} \longrightarrow \mathrm{X}$ be a plot and let $\mathrm{F}$ belong to $\mathrm{C}^{\infty}(\mathrm{V}, \mathrm{U})$, where $\mathrm{V}$ is any numerical domain, then $\mathrm{P} \circ \mathrm{F}$ is a plot.

A set equipped with a diffeology is called a diffeological space.

The first axiom implies that each point of $\mathrm{X}$ is covered by a plot. The second axiom clearly means that to be a plot is a local condition. And, the third axiom ensures some coherence of the use of the word differentiable in this context. The set of all plots of the diffeology $\mathcal{D}$ defined on a numerical domain $U$ will be denoted $\mathcal{D}(\mathrm{U}, \mathrm{X})$. Formally, a diffeological space is a pair $(X, \mathcal{D})$ where $\mathrm{X}$ is an arbitrary set and $\mathcal{D}$ a diffeology of $\mathrm{X}$. But most of the time the diffeological space will be denoted by the single letter $\mathrm{X}$ denoting its underlying space, the diffeology is understood.

Let us note however that the distinction between diffeology as a structure and diffeological space as a set together with a diffeology is psychological: the diffeology contains the underlying set as the set of 0-plots. As well, a topology contains the underlying space as the union of all open sets.

1.3. Standard diffeology of domains. The set of all smooth parametrizations of a numerical domain $\mathrm{U} \subset \mathbf{R}^{n}$ is clearly a diffeology. We shall call it the standard diffeology of $U$.

2. Differentiable maps. Diffeological spaces are the objects of a category whose morphisms are differentiable maps, and isomorphisms are the diffeomorphisms.

2.1. Differentiable maps and diffeomorphisms. Let $\mathrm{X}$ and $\mathrm{Y}$ be two diffeological spaces and $\mathrm{F}: \mathrm{X} \rightarrow \mathrm{Y}$ be a map. The map $\mathrm{F}$ is said to be differentiable if for each plot $\mathrm{P}$ of $\mathrm{X}, \mathrm{F} \circ \mathrm{P}$ is a plot of $\mathrm{Y}$. The set of differentiable maps from $\mathrm{X}$ to $\mathrm{Y}$ is denoted $\mathrm{e}^{\infty}(\mathrm{X}, \mathrm{Y})$. A bijective map $\mathrm{F}: \mathrm{X} \rightarrow \mathrm{Y}$ is said to be a diffeomorphism if both $\mathrm{F}$ and $\mathrm{F}^{-1}$ are differentiable. The set of all diffeomorphisms of $\mathrm{X}$ is a group denoted Diff(X).

2.2. The Diffeology category. The composition of differentiable maps is differentiable. Diffeological spaces, together with differentiable maps, define a category, denoted \{Diffeology\}. The isomorphisms of the category are diffeomorphisms.

2.3. Plots are smooth. The set of differentiable maps from a numerical domain $\mathrm{U}$ into a diffeological space $\mathrm{X}$ is exactly the set of plots of $\mathrm{X}$ defined on $\mathrm{U}$. This is a direct consequence of axiom D3. Hence, $\mathrm{C}^{\infty}(\mathrm{U}, \mathrm{X})=\mathcal{D}(\mathrm{U}, \mathrm{X})$ and we may equally use these two notations. This justifies, a posteriori, the use of the symbol $\mathrm{C}^{\infty}$ to denote the differentiable maps between diffeological spaces. And for this reason we may equally use the word smooth or the word differentiable. 
2.4. Comparing diffeologies. A large number of constructions in diffeology use the following relation on diffeologies: a diffeology $\mathcal{D}$ on a set $\mathrm{X}$ is said to be finer than another $\mathcal{D}^{\prime}$, if

$$
\mathcal{D} \subset \mathcal{D}^{\prime}
$$

The relation $\subset$ is a partial order on the diffeologies of any given set $\mathrm{X}$. We say indifferently that $\mathcal{D}$ is finer than $\mathcal{D}^{\prime}$ or $\mathcal{D}^{\prime}$ coarser than $\mathcal{D}$. Note that coarser means more plots and finer means fewer plots.

2.5. Discrete and coarse diffeologies. Any set $X$ carries a finest diffeology, finer than any other diffeology, called the discrete diffeology. The plots of the discrete diffeology are the locally constant parametrizations.

Any set X carries a coarsest diffeology, containing any other diffeology, it is called the coarse diffeology. The plots of the coarse diffeology are all parametrizations of $\mathrm{X}$, that is the whole set $\operatorname{Param}(\mathrm{X})$.

In these two cases, the three axioms of diffeology, covering, locality and smooth compatibility, are obviously satisfied. Any diffeology is somewhere between the discrete and the coarse diffeologies.

2.6. Intersecting diffeologies. As an illustration of the partial order on diffeologies, let us cite the following proposition. Let $\mathrm{X}$ be a set and $\mathbf{D}$ be any family of diffeologies of $\mathrm{X}$. The intersection

$$
\bigcap_{\mathcal{D} \in \mathbf{D}} \mathcal{D}
$$

is a diffeology. It is the coarsest diffeology contained in every element of $\mathbf{D}$, the finest being the discrete diffeology. This proposition is used to prove that every family of diffeologies has a supremum and an infimum. In other words, diffeologies form a lattice.

3. Generating families and dimension. Generating families are a convenient and useful tool in order to define a diffeology. They are defined by the following proposition.

3.1. Generating families. Let $\mathrm{X}$ be a set, let $\mathcal{F}$ be some subset of parametrizations of $\mathrm{X}$. There exists a finest diffeology containing $\mathcal{F}$. This diffeology will be called the diffeology generated by $\mathcal{F}$ and denoted $\langle\mathcal{F}\rangle$. This diffeology is the infimum (art. 2.6) of all diffeologies containing $\mathcal{F}$. Given a diffeological space $\mathrm{X}$, a family $\mathcal{F}$ generating the diffeology of $\mathrm{X}$ is called a generating family of $\mathrm{X}$. The plots of the diffeology generated by $\mathcal{F}$ are given by:

GF A parametrization $\mathrm{P}: \mathrm{U} \longrightarrow \mathrm{X}$ is a plot of the diffeology generated by $\mathcal{F}$ if and only if for every point $r$ of $\mathrm{U}$ there exists a superset $\mathrm{V} \subset \mathrm{U}$ of $r$ such that either $\mathrm{P} \uparrow \mathrm{V}$ is a constant parametrization, or there exists an element $\mathrm{Q}: \mathrm{W} \rightarrow \mathrm{X}$ of $\mathcal{F}$ and a smooth parametrization $\mathrm{F}: \mathrm{V} \rightarrow \mathrm{W}$ such that $\mathrm{P} \uparrow \mathrm{V}=\mathrm{Q} \circ \mathrm{F}$.

In the second case, we say that the plot $\mathrm{P}$ lifts locally along $\mathrm{F}$, or that $\mathrm{Q}$ is a local lifting of $\mathrm{P}$ along $\mathrm{F}$ (see fig. 1). Note that generating diffeologies is a projector, that is for any diffeology $\mathcal{D}$ we have $\langle\mathcal{D}\rangle=\mathcal{D}$.

3.2. Generated by the empty set. Note that, for any set $\mathrm{X}$, the empty family $\mathcal{F}=\varnothing$ generates the discrete diffeology. 


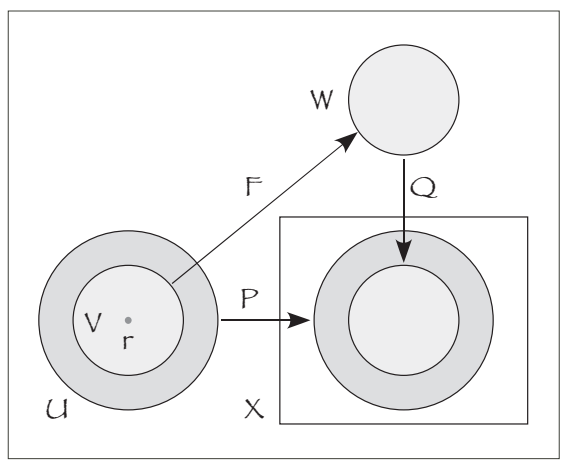

Fig. 1. Local lifting of $\mathrm{P}$ along $\mathrm{F}$

3.3. Dimension. Let $\mathrm{X}$ be a diffeological space and let $\mathcal{D}$ be its diffeology. Let us call dimension of a generating family $\mathcal{F}$ of $\mathcal{D}$ the supremum of the dimensions of the plots of the family:

$$
\operatorname{dim}(\mathcal{F})=\sup \{\operatorname{dim}(\mathrm{P}) \mid \mathrm{P} \in \mathcal{F}\},
$$

where $\operatorname{dim}(\mathrm{P})$ is defined in (art. 1.1). We define the dimension of the space $\mathrm{X}$ as the infimum of the dimensions of the generating families of the space:

$$
\operatorname{dim}(\mathrm{X})=\inf \{\operatorname{dim}(\mathcal{F}) \mid\langle\mathcal{F}\rangle=\mathcal{D}\} .
$$

Note that $\operatorname{dim}(\mathcal{F})$ as well as $\operatorname{dim}(\mathrm{X})$ can be either finite or infinite. For more details about dimension in diffeology see [PIZ06].

3.4. Dimensions of numerical domains. As we should expect, the dimension of a numerical domain $\mathrm{U} \subset \mathbf{R}^{n}$, equipped with the standard diffeology, is just $n$.

3.5. Differentiable maps via generating families. Let $\mathrm{X}$ be a diffeological space generated by a family $\mathcal{F}$ and $\mathrm{X}^{\prime}$ a diffeological space generated by a family $\mathcal{F}^{\prime}$. Let $f: \mathrm{X} \rightarrow \mathrm{X}^{\prime}$ be a map. The map $f$ is differentiable if and only if for each element $\mathrm{P}: \mathrm{U} \longrightarrow \mathrm{X}$ of $\mathcal{F}$, for each point $r$ of $\mathrm{U}$ there exists a superset $\mathrm{V}$ of $r$, an element $\mathrm{P}^{\prime}: \mathrm{U}^{\prime} \longrightarrow \mathrm{X}^{\prime}$ of $\mathcal{F}^{\prime}$ and a smooth parametrization $\mathrm{F}: \mathrm{V} \longrightarrow \mathrm{U}^{\prime}$ such that $f \circ \mathrm{P} \uparrow \mathrm{V}=\mathrm{P}^{\prime} \circ \mathrm{F}$. This is illustrated by the following diagram:

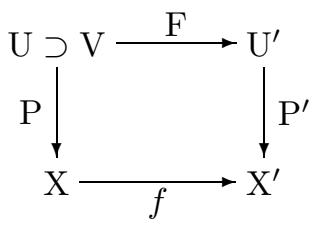

4. Pullbacks of diffeologies and inductions. The category $\{$ Diffeology $\}$ is stable under the subset operation. This stability is expressed by the following construction.

4.1. Pull-backs of diffeologies. Let $\mathrm{X}$ be a set, and $\mathrm{Y}$ be a diffeological space. Let $f: \mathrm{X} \longrightarrow$ $\mathrm{Y}$ be a map. There exists a coarsest diffeology on $\mathrm{X}$ such that the map $f$ is differentiable. This diffeology is called the pull-back diffeology. A parametrization $\mathrm{P}$ of $\mathrm{X}$ is a plot of 
the pull-back diffeology if and only if $f \circ \mathrm{P}$ is a plot of $\mathrm{Y}$. Let $\mathcal{D}$ be the diffeology on $\mathrm{Y}$, $f^{*}(\mathcal{D})$ will denote the pull-back diffeology of $\mathcal{D}$ by $f$.

$$
f^{*}(\mathcal{D})=\{\mathrm{P} \in \operatorname{Param}(\mathrm{X}) \mid f \circ \mathrm{P} \in \mathcal{D}\} .
$$

4.2. Compositions of pull-backs. Let $\mathrm{X}, \mathrm{Y}$ be two sets and $\mathrm{Z}$ be a diffeological space. Let $f: \mathrm{X} \rightarrow \mathrm{Y}$ and $g: \mathrm{Y} \rightarrow \mathrm{Z}$ be two maps. Let $\mathcal{D}$ be a diffeology on $\mathrm{Z}$, then $f^{*}\left(g^{*}(\mathcal{D})\right)=$ $(g \circ f)^{*}(\mathcal{D})$.

4.3. Inductions. Let $\mathrm{X}$ and $\mathrm{Y}$ be two diffeological spaces. $\mathrm{A}$ map $f: \mathrm{X} \rightarrow \mathrm{Y}$ is called an induction if $f$ is injective and if the pull-back diffeology of $\mathrm{Y}$ by $f$ coincides with the diffeology of $\mathrm{X}$. That is, the plots of $\mathrm{X}$ are the parametrizations $\mathrm{P}$ of $\mathrm{X}$ such that $f \circ \mathrm{P}$ are plots of Y.

4.4. Surjective inductions. Let $f: \mathrm{X} \rightarrow \mathrm{Y}$ be an injection, where $\mathrm{X}$ and $\mathrm{Y}$ are diffeological spaces. The map $f$ is an induction if and only if for any plot $\mathrm{P}$ of $\mathrm{Y}$, with values in $f(\mathrm{X})$, the map $f^{-1} \circ \mathrm{P}$ is a plot of $\mathrm{X}$. In particular, surjective inductions are diffeomorphisms.

4.5. Compositions of inductions. The composition of two inductions is again an induction. Inductions make up a subcategory of the category $\{$ Diffeology $\}$.

4.6. Subset diffeology and diffeological subspaces. Let $\mathrm{X}$ be a diffeological space. Any subset $\mathrm{A} \subset \mathrm{X}$ carries a natural diffeology induced by the inclusion. Namely the pull-back diffeology by the inclusion $j_{\mathrm{A}}: \mathrm{A} \hookrightarrow \mathrm{X}$ (art. 4.3). Equipped with this induced diffeology the subset $\mathrm{A}$ is called a subspace of $\mathrm{X}$. This diffeology is also called the subset diffeology. The plots of the subset diffeology of $\mathrm{A}$ are the plots of $\mathrm{X}$ taking their values in $\mathrm{A}$.

4.7. Sums of diffeological spaces. Let $\mathcal{X}$ be a family of diffeological spaces, there exists on the disjoint union $\amalg X$ of the elements of $X$ :

$$
\coprod x=\coprod_{\mathrm{X} \in X} \mathrm{x}
$$

a finest diffeology such that each injection $j_{\mathrm{X}}: \mathrm{X} \hookrightarrow \coprod X$ is differentiable. This diffeology is called the sum diffeology of the family $X$. The plots of the sum diffeology are the parametrizations $\mathrm{P}$ of $X$ which are locally plots of elements of the family $X$. In other words, a parametrization $\mathrm{P}: \mathrm{U} \longrightarrow \coprod \mathcal{X}$ is a plot of the sum diffeology if and only if there exists an open covering $\left\{\mathrm{U}_{i}\right\}_{i \in \mathcal{J}}$ of $\mathrm{U}$ and for each $i \in \mathcal{J}$ an element $\mathrm{X}_{i}$ of the family $\mathcal{X}$, such that $\mathrm{P} \uparrow \mathrm{U}_{i}$ is a plot of $\mathrm{X}_{i}$. For this diffeology, the injections $j_{\mathrm{X}}$ are inductions.

5. Push-forwards of diffeologies and subductions. The category $\{$ Diffeology $\}$ is stable by quotient, this stability is a consequence of the following construction.

5.1. Push-forward of diffeologies. Let $\mathrm{X}$ be a diffeological space. Let $\mathrm{Y}$ be a set and $f: \mathrm{X} \rightarrow \mathrm{Y}$ be a map. There exists a finest diffeology on $\mathrm{Y}$ such that $f$ is differentiable. This diffeology is called the push-forward (or image) of the diffeology of X. Let $\mathcal{D}$ be the diffeology of $\mathrm{X}$, the image of $\mathcal{D}$ by $f$ is denoted $f_{*}(\mathcal{D})$. A parametrization $\mathrm{P}: \mathrm{U} \rightarrow \mathrm{X}$ is an element of $f_{*}(\mathcal{D})$ if and only if for any $r \in \mathrm{U}$ there exists a superset $\mathrm{V}$ of $r$ such that either $\mathrm{P} \uparrow \mathrm{V}$ is a constant parametrization or there exists a plot $\mathrm{Q}: \mathrm{V} \rightarrow \mathrm{X}$ such that 
$\mathrm{P} \uparrow \mathrm{V}=f \circ \mathrm{Q}$. In other words, the diffeology $f_{*}(\mathcal{D})$ is generated (art. 3.1) by the plots of the form $f \circ \mathrm{Q}$ where $\mathrm{Q}$ is a plot of $\mathrm{X}$.

5.2. Subductions. Let $\mathrm{X}$ and $\mathrm{Y}$ be two diffeological spaces. A map $f: \mathrm{X} \rightarrow \mathrm{Y}$ is called a subduction if it is a surjection and if the image of the diffeology of $\mathrm{X}$ (art. 5.1) coincides with the diffeology of $\mathrm{Y}$. In this case, a parametrization $\mathrm{P}: \mathrm{U} \rightarrow \mathrm{Y}$ is a plot if and only if for any $r \in \mathrm{U}$ there exists a superset $\mathrm{V}$ of $r$ and a plot $\mathrm{Q}: \mathrm{V} \rightarrow \mathrm{X}$ such that $\mathrm{P} \uparrow \mathrm{V}=f \circ \mathrm{Q}$.

5.3. Quotients of diffeological spaces. Let $\mathrm{X}$ be a diffeological space and let $\sim$ be any equivalence relation on $\mathrm{X}$. The quotient space $\mathrm{Q}=\mathrm{X} / \sim$ carries a quotient diffeology, image of the diffeology of $\mathrm{X}$ by the projection $\pi: \mathrm{X} \rightarrow \mathrm{Q}$. A parametrization $\mathrm{P}: \mathrm{U} \rightarrow \mathrm{Q}$ is a plot of the quotient diffeology if and only if for any $r$ of $\mathrm{U}$ there exists a superset $\mathrm{V}$ of $r$ and a plot $\mathrm{P}^{\prime}: \mathrm{V} \longrightarrow \mathrm{X}$ such that $\mathrm{P} \uparrow \mathrm{V}=\pi \circ \mathrm{P}^{\prime}$.

5.4. Injective subductions. Injective subductions are diffeomorphisms.

5.5. Compositions of subductions. The composition of two subductions is again a subduction. Subductions make up a subcategory of the category $\{$ Diffeology $\}$.

5.6. Subquotients. Let $\mathrm{X}$ be a diffeological space, and let $\sim$ be an equivalence relation defined on $\mathrm{X}$. Let $\mathrm{A} \subset \mathrm{X}$ and let $\sim_{\mathrm{A}}$ be the restriction of $\sim$ to $\mathrm{A}$. Let $\mathcal{J}$ be the induction from $\mathrm{A}$ into $\mathrm{X}$, and let $j$ be the quotient map, defined from $\mathrm{A} / \sim_{\mathrm{A}}$ to $\mathrm{X} / \sim$. Let $\pi_{A}: \mathrm{A}$ $\rightarrow \mathrm{A} / \sim_{\mathrm{A}}$ and $\pi: \mathrm{X} \rightarrow \mathrm{X} / \sim$ be the projections onto the quotients.

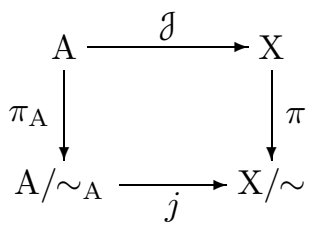

The subset $\mathrm{A}$ is equipped with the subset diffeology (art. 4.6), and the spaces $\mathrm{A} / \sim_{\mathrm{A}}$ and $\mathrm{X} / \sim$ are equipped with the quotient diffeology (art. 5.3). The map $j$ is a differentiable injection. It is an induction if and only if for any plot $\mathrm{P}: \mathrm{U} \rightarrow \mathrm{X}$, for any $r \in \mathrm{U}$, there exists a superset $\mathrm{V}$ of $r$ and a plot $\mathrm{Q}: \mathrm{V} \rightarrow \mathrm{A}$ such that $\pi \circ \mathrm{P} \uparrow \mathrm{V}=\pi \circ \mathrm{Q}$. This happens, in particular, if there exists a differentiable map $\rho: \mathrm{X} \rightarrow \mathrm{A}$ such that $\pi \circ \rho=\pi$. Moreover, in this case, if $\pi \uparrow \mathrm{A}$ is surjective then $j$ is a diffeomorphism.

This proposition will be used later to identify the infinite projective space, with the quotient of the infinite sphere by the action of $\mathrm{S}^{1}$ (art. 18.1).

5.7. Products of diffeological spaces. Let $\mathcal{X}$ be a family of diffeological spaces, there exists on the product

$$
\prod x=\prod_{\mathrm{x} \in X} \mathrm{x}
$$

a coarsest diffeology such that, for each $\mathrm{X}$ belonging to $X$ the projection $\pi_{\mathrm{X}}: \prod X \rightarrow \mathrm{X}$ is differentiable. This diffeology is called the product diffeology. The plots of this diffeology are the parametrizations $\mathrm{P}: \mathrm{U} \rightarrow \prod X$ such that for each $\mathrm{X} \in X, \pi_{\mathrm{X}} \circ \mathrm{P}$ is a plot of $\mathrm{X}$. For this diffeology the projections $\pi_{\mathrm{X}}$ are subductions. 
6. D-topology, locality, embeddings... Diffeologies are defined on arbitrary sets and are not subordinate to any extra structure. In particular they do not assume the existence of any underlying topology. But a set, when it is equipped with a diffeology, carries a natural topology, defined by compatibility with the diffeology. This topology is used to introduce locality into the diffeological framework. And it is through this construction that differential geometry of manifolds takes its place in the diffeology theory.

6.1. D-Topology. Let $\mathrm{X}$ be a diffeological space. There exists on $\mathrm{X}$ a finest topology such that the plots are continuous. This topology is called D-topology [Don84]. The open sets of the D-topology are the subsets $\mathrm{A} \subset \mathrm{X}$ such that for each plot $\mathrm{P}$ of $\mathrm{X}$, the pull-back $\mathrm{P}^{-1}(\mathrm{~A})$ is open. The open sets for the D-topology are called D-open.

6.2. Differentiable maps and continuity. Let $\mathrm{X}$ and $\mathrm{Y}$ be two diffeological spaces. Any differentiable map $f: \mathrm{X} \rightarrow \mathrm{Y}$ is continuous for the D-topology, one says that $f$ is D-continuous. In other words, considering the D-topology, $\mathrm{e}^{\infty}(\mathrm{X}, \mathrm{Y}) \subset \mathrm{e}^{0}(\mathrm{X}, \mathrm{Y})$.

6.3. D-topology on numerical domains. The D-topology of numerical domains, equipped with the standard diffeology (art. 1.3), coincides with the standard topology.

6.4. D-topology of discrete space. The D-topology of the discrete diffeology is the discrete topology. The D-topology of the coarse diffeology is the coarse topology. But, a non coarse diffeological space can inherit the coarse D-topology.

6.5. Quotients and D-topology. Let $\mathrm{X}$ be a diffeological space and $\sim$ be an equivalence relation defined on $\mathrm{X}$. The $\mathrm{D}$-topology of the quotient $\mathrm{X} / \sim$ is the quotient topology of the D-topology of X.

6.6. Embeddings. Let $\mathrm{A}$ be a subset of a diffeological space $\mathrm{X}$. The set A carries two natural topologies: its D-topology given by the subset diffeology, and the subset topology induced by the D-topology of the ambient space X. If these two topologies coincide we shall say that $\mathrm{A}$ is embedded in $\mathrm{X}$.

Note that, to be embedded depends only on the diffeology of the ambient space X, and not on any extra-structure, nor other diffeology. A subset of a diffeological space is embedded or not. For example, the set of rational numbers $\mathbf{Q} \subset \mathbf{R}$ is discrete (that is its subset diffeology is discrete), but not embedded in $\mathbf{R}$.

6.7. Local differentiability and differentiability. Let $\mathrm{X}$ and $\mathrm{Y}$ be two diffeological spaces. $\mathrm{A}$ map $f: \mathrm{A} \rightarrow \mathrm{Y}$ defined on a part $\mathrm{A} \subset \mathrm{X}$ is said to be locally differentiable if, for each plot $\mathrm{P}$ of $\mathrm{X}, f \circ \mathrm{P}$ is a plot of $\mathrm{Y}$. Note that $f \circ \mathrm{P}$ is defined on $\mathrm{P}^{-1}(\mathrm{~A})$ which is necessarily open if $f \circ \mathrm{P}$ is a plot. So, if $f: \mathrm{A} \longrightarrow \mathrm{X}$ is locally differentiable, $\mathrm{A}$ is necessarily $\mathrm{D}$-open, since for each plot $\mathrm{P}$ of $\mathrm{X}, \mathrm{P}^{-1}(\mathrm{~A})$ is a domain.

Let $f: \mathrm{X} \rightarrow \mathrm{Y}$ be a map between diffeological spaces. We shall say that $f$ is locally differentiable at the point $x \in \mathrm{X}$ if there exists a superset $\mathrm{V}$ of $x$ such that $f \uparrow \mathrm{V}$ is locally differentiable (which implies that $\mathrm{V}$ is $\mathrm{D}$-open). The map $f$ is differentiable if and only if it is locally differentiable at each point $x$ of $\mathrm{X}$.

6.8. Local diffeomorphisms. Let $\mathrm{X}$ and $\mathrm{Y}$ be two diffeological spaces. Let $f: \mathrm{A} \rightarrow \mathrm{Y}$ be a map defined on a part $\mathrm{A} \subset \mathrm{X}$. The map $f$ is said to be a local diffeomorphism if 
1. $f$ is injective,

2. $f$ is locally differentiable as well as $f^{-1}$, defined on $f(\mathrm{~A})$.

In this case, $\mathrm{A}$ and $f(\mathrm{~A})$ are both $\mathrm{D}$-open and $f: \mathrm{A} \rightarrow f(\mathrm{~A})$ is a diffeomorphism where A and $f(\mathrm{~A})$ are equipped with their subset diffeology. This is a necessary and sufficient condition for being a local diffeomorphism.

6.9. Diffeology is a local structure. There is another way to express the locality of diffeologies contained in the axiom of locality (art. 1.2). Let us consider a same set X equipped with two diffeologies $\mathcal{D}$ and $\mathcal{D}^{\prime}$. If there exists a D-open covering $\mathcal{U}$ of $\mathrm{X}$ such that the restricted diffeologies of $\mathcal{D}$ and $\mathcal{D}^{\prime}$ coincide on each element $U$ of $\mathcal{U}$, then the two diffeologies $\mathcal{D}$ and $\mathcal{D}^{\prime}$ coincide.

7. Functional diffeology. In the category $\{$ Diffeology $\}$ the spaces of differentiable maps between diffeological spaces are naturally diffeological spaces. This property is very convenient for many diffeological constructions, in particular — but not only for homotopy.

7.1. Functional diffeology. Let $\mathrm{X}$ and $\mathrm{Y}$ be two diffeological spaces. A functional diffeology on $\mathrm{C}^{\infty}(\mathrm{X}, \mathrm{Y})$ is a diffeology such that the map

$$
\text { ev }: \mathfrak{C}^{\infty}(\mathrm{X}, \mathrm{Y}) \times \mathrm{X} \rightarrow \mathrm{Y} \quad \text { with } \quad \mathbf{e v}(f, x)=f(x)
$$

is differentiable. For example, the discrete diffeology is a functional diffeology. However, there exists on $\mathrm{C}^{\infty}(\mathrm{X}, \mathrm{Y})$ a coarsest functional diffeology, called the standard functional diffeology, or simply the functional diffeology. A plot of the standard functional diffeology is any parametrization $\mathrm{P}: \mathrm{U} \longrightarrow \mathrm{C}^{\infty}(\mathrm{X}, \mathrm{Y})$ such that for any plot $\mathrm{Q}: \mathrm{V} \rightarrow \mathrm{X}$ the parametrization $\mathrm{P} \cdot \mathrm{Q}:(r, s) \mapsto \mathrm{P}(r)(\mathrm{Q}(s))$ is a plot of $\mathrm{Y}$. In particular, there exists a natural diffeomorphism between $\mathrm{C}^{\infty}\left(\mathrm{X}, \mathrm{e}^{\infty}(\mathrm{Y}, \mathrm{Z})\right)$ and $\mathrm{C}^{\infty}(\mathrm{X} \times \mathrm{Y}, \mathrm{Z})$, where $\mathrm{X}, \mathrm{Y}, \mathrm{Z}$ are any diffeological spaces.

7.2. Functional diffeology of groups of diffeomorphisms. Let $\mathrm{X}$ be a diffeological space and $\operatorname{Diff}(\mathrm{X})$ its group of diffeomorphisms. The standard functional diffeology has a specialization in the case of $\operatorname{Diff}(\mathrm{X}) \subset \mathrm{C}^{\infty}(\mathrm{X}, \mathrm{X})$. A group diffeology (art. 11.1) is a diffeology such that the multiplication and the inversion are differentiable. Now, there exists a coarsest group diffeology on $\operatorname{Diff}(\mathrm{X})$ such that the function ev (art. 7.1) is differentiable, it is called the standard functional diffeology of Diff $(\mathrm{X})$. A parametrization $\mathrm{P}: \mathrm{U}$ $\longrightarrow \operatorname{Diff}(\mathrm{X})$ is a plot of this diffeology if and only if for any plot $\mathrm{Q}: \mathrm{V} \rightarrow \mathrm{X}$ the two maps $(r, s) \mapsto \mathrm{P}(r)(\mathrm{Q}(s))$ and $(r, s) \mapsto \mathrm{P}(r)^{-1}(\mathrm{Q}(s))$ are plots of $\mathrm{X}$.

8. Homotopy. The traditional theory of homotopy extends naturally to diffeological spaces. This paragraph presents just the constructions of the homotopy groups of diffeological spaces. For more details on homotopy in diffeology see [Igl85].

8.1. Connected components of diffeological spaces. Let $\mathrm{X}$ be a diffeological space, we denote by Paths(X) the set of global 1-plots of $\mathrm{X}$

$$
\operatorname{Paths}(\mathrm{X})=\mathrm{e}^{\infty}(\mathbf{R}, \mathrm{X}),
$$


equipped with the functional diffeology. The relation of homotopy or connectedness is defined on X by:

$$
x \sim x^{\prime} \quad \Leftrightarrow \quad \text { there exists } \gamma \in \operatorname{Paths}(\mathrm{X}) \text { such that } \gamma(0)=x \text { and } \gamma(1)=x^{\prime} .
$$

If $x \sim x^{\prime}$ we say that $x$ is homotopic to $x^{\prime}$ or connected to $x^{\prime}$. To be homotopic is an equivalence relation. A class of this relation is called a connected component of $\mathrm{X}$. The class, or the connected component, of $x \in \mathrm{X}$ is denoted $[x]$. The space of connected components of $\mathrm{X}$, denoted $\pi_{0}(\mathrm{X})$, is the quotient of $\mathrm{X}$ by the relation of homotopy:

$$
\pi_{0}(\mathrm{X})=\mathrm{X} / \sim
$$

Equipped with the quotient diffeology, the space $\pi_{0}(\mathrm{X})$ is discrete. More precisely, the partition into connected components is the finest partition of $\mathrm{X}$ such that $\mathrm{X}$ is the sum (art. 4.7) of its parts. The pointed space $\pi_{0}\left(\mathrm{X}, x_{0}\right)$, where $x_{0} \in \mathrm{X}$, is defined as

$$
\pi_{0}\left(\mathrm{X}, x_{0}\right)=\left(\pi_{0}(\mathrm{X}),\left[x_{0}\right]\right)
$$

If $\pi_{0}(\mathrm{X})=\left\{\left[x_{0}\right]\right\}$ for some $x_{0} \in \mathrm{X}$, the space $\mathrm{X}$ is said to be connected.

8.2. Iterated loop spaces and higher homotopy. Let $\mathrm{X}$ be any diffeological space and $x_{0} \in \mathrm{X}$. The space of loops based at $x_{0}$ is defined as:

$$
\operatorname{Loops}\left(\mathrm{X}, x_{0}\right)=\left\{\gamma \in \operatorname{Paths}(\mathrm{X}) \mid \gamma(0)=\gamma(1)=x_{0}\right\} \text {. }
$$

The space Loops $\left(\mathrm{X}, x_{0}\right)$ is equipped with the functional diffeology. The higher homotopy spaces are defined by recursion:

$$
\pi_{k}\left(\mathrm{X}, x_{0}\right)=\pi_{k-1}\left(\operatorname{Loops}\left(\mathrm{X}, x_{0}\right), \hat{x}_{0}\right), \quad x_{0} \in \mathrm{X}, \quad \hat{x}_{0}=\left[t \mapsto x_{0}\right] .
$$

In particular, the space

$$
\pi_{1}\left(\mathrm{X}, x_{0}\right)=\pi_{0}\left(\operatorname{Loops}\left(\mathrm{X}, x_{0}\right), \hat{x}_{0}\right)
$$

is called the fundamental group of $\mathrm{X}$, based at $x_{0}$. Juxtaposition of loops, described below (art. 8.3), gives $\pi_{1}\left(\mathrm{X}, x_{0}\right)$ a structure of group. If $\mathrm{X}$ is connected and $\pi_{1}\left(\mathrm{X}, x_{0}\right)=\left\{\left[\hat{x}_{0}\right]\right\}$ the space $\mathrm{X}$ is said to be 1-connected, or connected and simply connected.

8.3. Homotopy group multiplication. Let $\mathrm{X}$ be a diffeological space and let $x_{0} \in \mathrm{X}$. Let $\gamma$ and $\gamma^{\prime}$ be two loops, based at $x_{0}$. The juxtaposition of $\gamma$ with $\gamma^{\prime}$ is defined traditionally by:

$$
\gamma \vee \gamma^{\prime}= \begin{cases}\gamma(2 t) & \text { if } t<1 / 2, \\ \gamma^{\prime}(2 t-1) & \text { if } 1 / 2<t\end{cases}
$$

Then, the group operation on the homotopy groups is defined on the class of loops by:

$$
[\gamma] \cdot\left[\gamma^{\prime}\right]=\left[\gamma \vee \gamma^{\prime}\right]
$$

But $\gamma \vee \gamma^{\prime}$ is, not necessarily a path, that is, not necessarily differentiable. So, we « smash » $\gamma$ and $\gamma^{\prime}$ at their ends, composing them with a «smashing function $» \varepsilon$, described by figure 2 .

The smashing function $\varepsilon$ is a smooth real function, homotopic to the identity of $\mathbf{R}$, sending an open superset of $]-\infty, 0]$ to 0 and an open superset of $[1, \infty[$ to 1 . Hence, the juxtaposition of the paths $\tilde{\gamma}=\gamma \circ \varepsilon$ and $\tilde{\gamma}^{\prime}=\gamma^{\prime} \circ \varepsilon$ is now a (differentiable) path with 


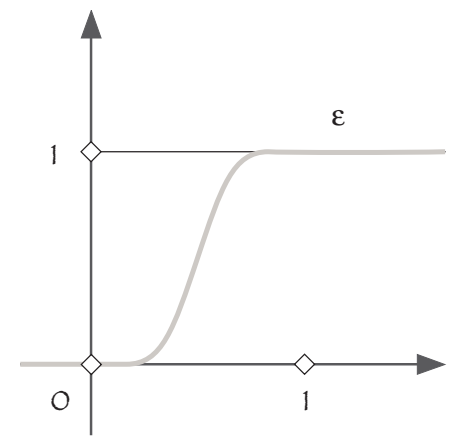

Fig. 2. The smashing function $\varepsilon$

the same ends as $\gamma \vee \gamma^{\prime}$. Now, since for any path $\gamma$ or $\gamma^{\prime}$, the smashed path is homotopic to the path itself, the operation defined on the classes of loops $[\gamma]$ and $\left[\gamma^{\prime}\right]$ by

$$
[\gamma] \cdot\left[\gamma^{\prime}\right]=\left[\tilde{\gamma} \vee \tilde{\gamma}^{\prime}\right]
$$

is well defined. As usual, we can check that this composition is a group operation: its identity is the class of the constant path $\hat{x}_{0}: t \mapsto x_{0}$, and the inverse of the class $[\gamma]$ is the class $[\gamma]^{-1}=[t \mapsto \gamma(1-t)]$. The space $\pi_{1}\left(\mathrm{X}, x_{0}\right)$ is always considered equipped with this group structure.

Now let us introduce the following iterated spaces: For all $k>1$,

$$
\operatorname{Loops}^{k}\left(\mathrm{X}, x_{0}\right)=\operatorname{Loops}^{k-1}\left(\operatorname{Loops}\left(\mathrm{X}, x_{0}\right), \hat{x}_{0}^{k-1}\right) \quad \text { and } \quad \hat{x}_{0}^{k}: t \mapsto \hat{x}_{0}^{k-1},
$$

initialized by:

$$
\operatorname{Loops}^{1}\left(\mathrm{X}, x_{0}\right)=\operatorname{Loops}\left(\mathrm{X}, x_{0}\right) \quad \text { and } \quad \hat{x}_{0}^{1}: t \mapsto \hat{x}_{0} .
$$

So, the recursion defined in (art. 8.2), gives in particular for $k \geq 1$ :

$$
\pi_{k}\left(\mathrm{X}, x_{0}\right)=\pi_{0}\left(\operatorname{Loops}^{k}\left(\mathrm{X}, x_{0}\right), \hat{x}_{0}^{k}\right) \quad \text { and } \quad \pi_{k}\left(\mathrm{X}, x_{0}\right)=\pi_{1}\left(\operatorname{Loops}^{k-1}\left(\mathrm{X}, x_{0}\right), \hat{x}_{0}^{k-1}\right) .
$$

Now, any higher homotopy space $\pi_{k}\left(\mathrm{X}, x_{0}\right), k \geq 1$, is a group since it is the fundamental group of some intermediate loop space. Moreover, as in the usual theory of homotopy, for $k \geq 2$ we check that $\pi_{k}\left(\mathrm{X}, x_{0}\right)$ is abelian [Igl85].

8.4. Contractibility. A diffeological space is said to be contractible if the identity map $\mathbf{1}_{\mathrm{X}}=[x \mapsto x]$ is homotopic (art. 8.1) to some constant map $\boldsymbol{x}_{0}=\left[x \mapsto x_{0}\right]$, with $x_{0} \in \mathrm{X}$, and $\mathrm{C}^{\infty}(\mathrm{X}, \mathrm{X})$ equipped with the standard functional diffeology. Note that if $\mathrm{X}$ is contractible any homotopy group is trivial: $\pi_{k}(\mathrm{X})=\{\star\}$.

9. Diffeological fibrations. Fiber bundles in the category $\{$ Diffeology $\}$ and their properties are detailed in [Igl85]. Diffeological fibrations are defined below, they are projections satisfying a property of local triviality along plots. Note that this definition, even if it coincides with the standard definition for finite dimensional manifolds, is more flexible than its topological analogue which requires local triviality. For example, any quotient $\mathrm{G} / \mathrm{H}$ of a diffeological group $\mathrm{G}$ (art. 11.1) by a subgroup $\mathrm{H}$ is a diffeological fibration with fiber H. But these fibrations $\mathrm{G} \longrightarrow \mathrm{G} / \mathrm{H}$ are not always locally trivial, for the D-topology, as 
many examples show. The following is a limited and pedestrian reminder of the main constructions.

9.1. Diffeological fiber bundles. Let $\pi: \mathrm{T} \rightarrow \mathrm{B}$ be a map between diffeological spaces. We shall say that $\pi$ is a diffeological fibration if there exists a diffeological space $\mathrm{F}$ such that for any plot $\mathrm{P}: \mathrm{U} \longrightarrow \mathrm{B}$ the pull-back $\mathrm{P}^{*}(\mathrm{~T})$ defined by

$$
\mathrm{P}^{*}(\mathrm{~T})=\{(r, t) \in \mathrm{U} \times \mathrm{T} \mid \mathrm{P}(r)=\pi(t)\}
$$

is locally trivial over $\mathrm{U}$, with fiber F. More precisely, for any $r \in \mathrm{U}$ there exists an open superset $\mathrm{V} \subset \mathrm{U}$ of $r$, a diffeomorphism $\Phi: \mathrm{V} \times \mathrm{F} \rightarrow(\mathrm{P} \uparrow \mathrm{V})^{*}(\mathrm{~T})$ such that $\operatorname{pr}_{1}(\Phi(r, f))=r$, where $\mathrm{pr}_{1}$ is the first projection from $(\mathrm{P} \uparrow \mathrm{V})^{*}(\mathrm{~T})$ onto $\mathrm{V}$. The space $\mathrm{F}$ is called the fiber. We say that $\pi$ is locally trivial along the plots.

Note that this definition involves, indirectly, the functional diffeology of the group of diffeomorphisms (art. 7.2) of F, but we shall not develop this aspect here, for a comprehensive report see [Igl85]. Note also that a diffeological fibration is a fortiori a subduction (art. 5.2).

9.2. Bundles and homotopy. Fiber bundles are not necessarily locally trivial for the D-topology, for example this fails for irrational fibrations of tori [Igl85]. However, it can happen that diffeological fiber bundles are also locally trivial. This is the case for the infinite Hopf fibration (art. 18.1). But, despite this lack of local triviality, any diffeological fiber bundle satisfies the homotopy exact sequence [Igl85] :

$$
\cdots \rightarrow \pi_{k+1}\left(\mathrm{~B}, b_{0}\right) \rightarrow \pi_{k}\left(\mathrm{~F}, t_{0}\right) \rightarrow \pi_{k}\left(\mathrm{~T}, t_{0}\right) \rightarrow \pi_{k}\left(\mathrm{~B}, b_{0}\right) \rightarrow \cdots \rightarrow\{0\}
$$

where $\pi: \mathrm{T} \rightarrow \mathrm{B}$ is a diffeological fibration, $b_{0} \in \mathrm{B}, \pi\left(t_{0}\right)=b_{0}$ and $\mathrm{F}=\pi^{-1}\left(b_{0}\right)$.

10. Differential calculus in diffeology. As a final structure, diffeologies support a well defined, and easy to use, notion of differential forms. They are defined by functoriality with differential forms of numerical domains.

10.1. Differential forms. Let $\mathrm{X}$ be a diffeological space, a $k$-form on $\mathrm{X}$ is any map $\alpha$ which associates to any plot $\mathrm{P}: \mathrm{U} \longrightarrow \mathrm{X}$ a $k$-form defined on $\mathrm{U}$, denoted by $\alpha(\mathrm{P})$, such that for any numerical domain $\mathrm{V}$ and any smooth parametrization $\mathrm{F}: \mathrm{V} \rightarrow \mathrm{U}$,

$$
\alpha(\mathrm{P} \circ \mathrm{F})=\mathrm{F}^{*}(\alpha(\mathrm{P})) .
$$

The $k$-form $\alpha(\mathrm{P})$ and the pull-back $\mathrm{F}^{*}(\alpha(\mathrm{P}))$ are understood as usual. We denote by $\Omega^{k}(\mathrm{X})$ the vector space of $k$-forms on $\mathrm{X}$. In the same spirit as for differentiable maps, there exists on any space $\Omega^{k}(\mathrm{X})$ a functional diffeology [PIZ05] such that addition and multiplication by a scalar are differentiable.

10.2. Exterior differential of a form. Many usual constructions on differential forms have a natural generalization to diffeological spaces. The exterior differential is an example among many. Let $\alpha$ be a $k$-form on $\mathrm{X}$. The exterior differential $d \alpha$ is defined by:

$$
(d \alpha)(\mathrm{P})=d(\alpha(\mathrm{P})),
$$

for any plot $\mathrm{P}$ of $\mathrm{X}$. This definition gives rise to the de Rham cohomology, defined as usual by

$$
\mathrm{H}_{\mathrm{dR}}^{*}(\mathrm{X})=\mathrm{Z}_{\mathrm{dR}}^{*}(\mathrm{X}) / \mathrm{B}_{\mathrm{dR}}^{*}(\mathrm{X})
$$


where $\mathrm{Z}_{\mathrm{dR}}^{*}(\mathrm{X})$ and $\mathrm{B}_{\mathrm{dR}}^{*}(\mathrm{X})$ denote respectively the subspace of closed and exact differential forms of $\mathrm{X}$.

For example [DI85] the de Rham cohomology of the irrational torus $\mathrm{T}_{\alpha}=\mathbf{R} /(\mathbf{Z}+\alpha \mathbf{Z})$ is

$$
\mathrm{H}_{\mathrm{dR}}^{k}\left(\mathrm{~T}_{\alpha}\right)=\{0\} \text { if } k \neq 1 \text { and } \mathrm{H}_{\mathrm{dR}}^{1}\left(\mathrm{~T}_{\alpha}\right)=\mathbf{R} .
$$

10.3. Pullbacks of differential forms. Let $\mathrm{X}$ and $\mathrm{Y}$ be two diffeological spaces. Let $f: \mathrm{X}$ $\longrightarrow \mathrm{Y}$ be a differentiable map, let $\alpha$ be a $k$-form on $\mathrm{Y}$, then the pull-back of $\alpha$ by $f$ is defined by:

$$
\left[f^{*}(\alpha)\right](\mathrm{P})=\alpha(f \circ \mathrm{P})
$$

for all plots $\mathrm{P}$ of $\mathrm{X}$. We check easily that $f^{*}(\alpha)$ is a well defined $k$-form on $\mathrm{X}$. This last definition justifies the following notation, or interpretation:

$$
\mathrm{P}^{*}(\alpha)=\alpha(\mathrm{P}) \text {. }
$$

And the form $\alpha(\mathrm{P}) \in \Omega^{k}(\mathrm{U})$, where $\mathrm{P}: \mathrm{U} \rightarrow \mathrm{X}$ is a plot, can be interpreted as the « coordinates » of the form $\alpha$ in the plot $\mathrm{P}$.

10.4. Locality of forms. Let $\mathrm{X}$ be a diffeological space. Let $\left(\mathrm{X}_{i}\right)_{i \in \mathcal{J}}$ be a D-open cover of $\mathrm{X}$, that is an open cover for the D-topology (art. 6.1). Let $\left(\alpha_{i}\right)_{i \in \mathcal{J}}$ be a family of $k$-forms such that:

1. For every index $i, \alpha_{i}$ is a $k$-form on $\mathrm{X}_{i}$, equipped with the subset diffeology (art. 4.6).

2. For every pair $i$ and $j$ of indices, $\alpha_{i} \uparrow \mathrm{X}_{i} \cap \mathrm{X}_{j}=\alpha_{j} \uparrow \mathrm{X}_{i} \cap \mathrm{X}_{j}$.

Then, there exists a unique $k$-form $\alpha$ on $\mathrm{X}$ such that $\alpha_{i}=\alpha \uparrow \mathrm{X}_{i}$.

10.5. Equality of forms. Let $\alpha$ and $\beta$ be two $p$-forms defined on a diffeological space X, $\alpha=\beta$ if and only if, for every $p$-plot $\mathrm{P}$ of $\mathrm{X}: \alpha(\mathrm{P})=\beta(\mathrm{P})$. In particular, a $p$-form vanishes identically if and only if it vanishes on every $p$-plot. Although, if $p$-forms are characterized by their values on $p$-plots, their differentiability is ensured by the differentiability of their values on every plot and not only on the $p$-plots.

10.6. Invariant forms and automorphisms of a form. Let $\mathrm{X}$ be a diffeological space and $\alpha$ a $k$-form on $\mathrm{X}$, let $f$ be a diffeomorphism of $\mathrm{X}$; we say that $\alpha$ is invariant by $f$ if $f^{*}(\alpha)=\alpha$. In other words, the form $\alpha$ is invariant by $f$ if and only if, for any plot $\mathrm{P}$ of $\mathrm{X}, \alpha(\mathrm{P})=\alpha(f \circ \mathrm{P})$. The set of all automorphisms of a form $\alpha$, denoted by

$$
\operatorname{Aut}(\alpha)=\left\{f \in \operatorname{Diff}(\mathrm{X}) \mid f^{*}(\alpha)=\alpha\right\}
$$

is a group. This group is also called the group of symmetries of the form $\alpha$. Equipped with the functional diffeology, $\operatorname{Aut}(\alpha)$ is a diffeological group in the natural sense given below (art. 11.1).

11. Diffeological groups and moments. Diffeological groups were first introduced as 《 groupes différentiels» in the early '80s [Sou81, Sou84]. They are to diffeological spaces what Lie groups are to manifolds. We recall here their definition. Then, we propose [PIZ05] as an equivalent to the "dual of the Lie algebra", the space of invariant 1-forms of the group. We don't consider any duality with a putative diffeological Lie algebra. This is the correct way to talk about coadjoint action or coadjoint orbits in diffeology. 
11.1. Diffeological groups. Let $\mathrm{G}$ be a group equipped with a diffeology. We say that $\mathrm{G}$ is a diffeological group if multiplication as well as inversion are differentiable:

$$
\left[\left(g, g^{\prime}\right) \mapsto g g^{\prime}\right] \in \mathcal{C}^{\infty}(\mathrm{G} \times \mathrm{G}, \mathrm{G}) \quad \text { and } \quad\left[g \mapsto g^{-1}\right] \in \mathcal{C}^{\infty}(\mathrm{G}) .
$$

Note that if $\mathrm{G}$ is a finite dimensional manifold this definition is nothing but the definition of Lie groups. We denote by $\mathrm{L}(g)$ and $\mathrm{R}(g)$ the left and right actions of $\mathrm{G}$ on itself.

$$
\text { For all } g \in \mathrm{G}, \quad\left\{\begin{array}{l}
\mathrm{L}(g): g^{\prime} \mapsto g g^{\prime} \\
\mathrm{R}(g): g^{\prime} \mapsto g^{\prime} g
\end{array}\right.
$$

Note that the «right action » is in fact an anti-action. The adjoint action of G onto itself is denoted Ad and defined by:

$$
\text { For all } g \in \mathrm{G}, \quad \operatorname{Ad}(g): k \mapsto g k g^{-1}=\mathrm{L}(g) \circ \mathrm{R}\left(g^{-1}\right)(k) .
$$

11.2. Moments of a diffeological group. We call moment (or momentum) of a diffeological group $\mathrm{G}$ any 1-form on $\mathrm{G}$, invariant by the left action. We denote by $\mathcal{G}^{*}$ the space of moments of $\mathrm{G}$. The space of moments of a diffeological group is naturally a vector space.

$$
\mathcal{G}^{*}=\left\{\alpha \in \Omega^{1}(\mathrm{G}) \mid \text { For all } g \in \mathrm{G}, \mathrm{L}(g)^{*}(\alpha)=\alpha\right\} .
$$

There exists a natural isomorphism between the space of left-invariant 1-forms and rightinvariant 1-forms [PIZ05].

Note that in spite of what the notation $\mathcal{G}^{*}$ suggests, the space of moments of a diffeological group is not defined by some duality. This notation is chosen here just to remind us the connection with the dual of the Lie algebra in the case of Lie groups.

11.3. Coadjoint action of $\mathrm{G}$ on $\mathcal{G}^{*}$ and coadjoint orbits. The pull-back of a moment $\alpha \in \mathcal{G}^{*}$ by the adjoint action of $\mathrm{G}$ is still a moment of $\mathrm{G}$, that is still a left-invariant 1-form. This defines a linear action of $\mathrm{G}$ on $\mathcal{G}^{*}$ called the linear coadjoint action. We shall denote:

$$
\text { For all } g \in \mathrm{G}, \text { for all } \alpha \in \mathcal{G}^{*}, \quad \operatorname{Ad}^{*}(g)(\alpha)=\operatorname{Ad}\left(g^{-1}\right)^{*}(\alpha) .
$$

And one checks that it is indeed an action of $\mathrm{G}$ :

$$
\text { For all } g, g^{\prime} \in \mathrm{G}, \quad \operatorname{Ad}^{*}\left(g g^{\prime}\right)=\operatorname{Ad}^{*}(g) \circ \operatorname{Ad}^{*}\left(g^{\prime}\right) \text {. }
$$

Note that, since $\alpha$ is left-invariant, $\operatorname{Ad}^{*}(g)(\alpha)=\mathrm{R}(g)^{*}(\alpha)$. Let $\alpha$ be a moment of $\mathrm{G}$, the orbit of $\alpha$ by $\mathrm{G}$ is, by definition, a linear coadjoint orbit of $\mathrm{G}$. And it will be denoted:

$$
\mathcal{O}_{\alpha} \text { or } \operatorname{Ad}^{*}(\mathrm{G})(\alpha)=\left\{\operatorname{Ad}^{*}(g)(\alpha) \mid g \in \mathrm{G}\right\} .
$$

The orbit $\mathcal{O}_{\alpha}$ can be viewed also as the quotient of the group $\mathrm{G}$ by the stabilizer of the moment $\alpha$,

$$
\mathcal{O}_{\alpha} \simeq \mathrm{G} / \operatorname{St}_{\mathrm{G}}(\alpha) \text {, with } \operatorname{St}_{\mathrm{G}}(\alpha)=\left\{g \in \mathrm{G} \mid \mathrm{R}(g)^{*}(\alpha)=\alpha\right\}
$$

The orbit $\mathcal{O}_{\alpha}$ will be equipped in the following with this quotient diffeology.

Note that the vector space $\mathcal{G}^{*}$ carries a functional diffeology [PIZ05] which induces on $\mathcal{O}_{\alpha}$ a subset functional diffeology. There is no reason a priori that these two diffeologies coincide. But it could be interesting however to understand in which conditions they do. 


\section{DIFFEOLOGICAL VECTOR SPACES AND MANIFOLDS}

We consider the fields of real numbers $\mathbf{R}$ and complex numbers $\mathbf{C}$, equipped with their standard diffeologies. The field $\mathbf{C}$ is diffeologically equivalent to $\mathbf{R}^{2}$. The natural map $(x, y) \mapsto z=x+i y$, from $\mathbf{R}^{2}$ to $\mathbf{C}$, is a diffeomorphism of diffeological space. In other words, a plot of $\mathbf{C}$ is just a parametrization $r \mapsto \mathrm{P}(r)+i \mathrm{Q}(r)$ where $\mathrm{P}$ and $\mathrm{Q}$ are smooth real parametrizations. In the following, the letter $\mathbf{K}$ denotes $\mathbf{R}$ or $\mathbf{C}$.

\section{Basic constructions and definitions}

12.1. Diffeological vector spaces. Let $\mathrm{E}$ be a vector space over $\mathbf{K}$, we call vector space diffeology on $\mathrm{E}$, any diffeology of $\mathrm{E}$ such that addition $(u, v) \mapsto u+v$, and multiplication by a scalar $(\lambda, u) \mapsto \lambda u$, are differentiable, where the spaces $\mathrm{E} \times \mathrm{E}$ and $\mathbf{K} \times \mathrm{E}$ are equipped with the product diffeology (art. 5.7). The space E, equipped with a vector space diffeology, is called a diffeological vector space.

12.2. Finite dimensional vector spaces. Finite dimensional vector spaces, over $\mathbf{R}$ or $\mathbf{C}$, equipped with their standard diffeology are diffeological vector spaces. But, note that any vector space equipped with the coarse diffeology is also a diffeological vector space.

12.3. Example: scalar differentiable maps. Let $\mathrm{X}$ be a diffeological space, and $\mathrm{E}=$ $\mathrm{e}^{\infty}\left(\mathrm{X}, \mathbf{K}^{n}\right)$, the space of differentiable maps from $\mathrm{X}$ to $\mathbf{K}^{n}$. The space $\mathrm{E}$ is naturally a $\mathbf{K}$-vector space for pointwise addition and multiplication by a scalar. Equipped with the functional diffeology, $\mathrm{E}$ is a diffeological $\mathbf{K}$-vector space.

12.4. Products and quotients of diffeological vector spaces. The product of any family of diffeological vector spaces is a diffeological vector space for the product diffeology. As well, the quotient of any diffeological vector space by any subspace is a diffeological vector space for the quotient diffeology.

Proof. Let us consider the product $\mathbf{E}=\prod_{\mathrm{E} \in \mathcal{E}} \mathrm{E}$ of a family $\mathcal{E}$ of diffeological vector spaces. The elements of $\mathbf{E}$ are the families $\boldsymbol{x}=\left(x_{\mathrm{E}}\right)_{\mathrm{E} \in \mathcal{E}}$, where the $x_{\mathrm{E}}$ are elements of $\mathrm{E}$. The sum and the product are defined by: $\boldsymbol{x}+\boldsymbol{x}^{\prime}=\left(x_{\mathrm{E}}\right)_{\mathrm{E} \in \mathcal{E}}+\left(x_{\mathrm{E}}^{\prime}\right)_{\mathrm{E} \in \mathcal{E}}=\left(x_{\mathrm{E}}+x_{\mathrm{E}}^{\prime}\right)_{\mathrm{E} \in \mathcal{E}}$, and $\lambda \boldsymbol{x}=\lambda\left(x_{\mathrm{E}}\right)_{\mathrm{E} \in \mathcal{E}}=\left(\lambda x_{\mathrm{E}}\right)_{\mathrm{E} \in \mathcal{E}}$. A parametrization $\mathrm{P}: \mathrm{U} \rightarrow \mathbf{E}$ is a plot if, for every $\mathrm{E}$ in $\mathcal{E}$, the parametrization $\mathrm{P} \circ \pi_{\mathrm{E}}$ is a plot of $\mathrm{E}$, where $\pi_{E}$ is the projection from $\mathbf{E}$ onto its factor (art. 5.7). Since addition and multiplication are differentiable on every factor of $\mathbf{E}$, it is clear that they are differentiable on $\mathbf{E}$.

12.5. Differentiable linear maps and category. Let $\mathrm{E}$ and $\mathrm{F}$ be two diffeological vector spaces over $\mathbf{K}$. Addition of linear maps from $\mathrm{E}$ to $\mathrm{F}$, as well as multiplication by a scalar are differentiable. As an immediate consequence, the space $\mathrm{LC}^{\infty}(\mathrm{E}, \mathrm{F})$ of $\mathbf{K}$-linear differentiable maps from $\mathrm{E}$ to $\mathrm{F}$ is a $\mathbf{K}$-vector subspace of $\mathrm{L}(\mathrm{E}, \mathrm{F})$.

Diffeological vector spaces, together with differentiable linear maps, form a category, the diffeological linear category. Isomorphisms of this category are bi-differentiable linear isomorphisms. 
13. Fine diffeology of vector spaces. Any $\mathbf{K}$-vector space equipped with the coarse diffeology is obviously a diffeological vector space, which is not really interesting. But also, any vector space has a finest vector space diffeology. In this section we analyze some aspects of this fine diffeology.

13.1. Fine diffeology. There exists, on any vector space $\mathrm{E}$ over the field $\mathbf{K}$ a finest diffeology of vector space. We shall call it the fine diffeology. This diffeology is generated by the family of parametrizations defined by:

$$
\mathrm{P}: r \mapsto \sum_{\alpha \in \mathrm{A}} \lambda_{\alpha}(r) v^{\alpha}
$$

where $\mathrm{A}$ is a finite set of indices, $\lambda_{\alpha}$ are smooth $\mathbf{K}$-functions defined on the domain of $\mathrm{P}$ and $v^{\alpha}$ are vectors of $\mathrm{E}$.

More precisely, the plots of the fine diffeology are the parametrizations $\mathrm{P}: \mathrm{U} \longrightarrow \mathrm{E}$ such that for any $r_{0} \in \mathrm{U}$ there exists a superset $\mathrm{V}$ of $r_{0}$, a family of smooth parametrizations $\lambda_{\alpha}: \mathrm{V} \rightarrow \mathbf{K}$ and a family of vectors $v^{\alpha} \in \mathrm{E}$, both indexed by the same finite set of indices $\mathrm{A}$, such that:

$$
(\mathrm{P} \uparrow \mathrm{V}): r \mapsto \sum_{\alpha \in \mathrm{A}} \lambda_{\alpha}(r) v^{\alpha} .
$$

The family $\left(\lambda_{\alpha}, v^{\alpha}\right)_{\alpha \in \mathrm{A}}$ such that $\lambda_{\alpha} \in \mathcal{C}^{\infty}(\mathrm{V}, \mathbf{K})$ and $v^{\alpha} \in \mathrm{E}$ will be called in the following a local family of the plot $\mathrm{P}$, or simply a local family.

Proof. Let us prove that the parametrizations described by $\diamond$ make up a diffeology of vector space.

1) Diffeology. Constant parametrizations satisfy the condition above. The locality is satisfied by definition. Now, let $\mathrm{F}: \mathrm{U}^{\prime} \longrightarrow \mathrm{U}$ be a smooth parametrization, we have just to change $\lambda_{\alpha}$ to $\lambda_{\alpha} \circ \mathrm{F}$, with the same $v^{\alpha}$, and $\mathrm{P} \circ \mathrm{F}$ satisfies the condition of the definition above. So, the set of parametrizations defined above is a diffeology.

2) Diffeology of vector space. Let $r \mapsto(\mathrm{P}(r), \mathrm{Q}(r))$ be a plot of the product $\mathrm{E} \times \mathrm{E}$. Let $\left(\lambda_{\alpha}, u^{\alpha}\right)_{\alpha \in \mathrm{A}}$ and $\left(\mu_{\beta}, v^{\beta}\right)_{\beta \in \mathrm{B}}$ be two local families such that locally:

$$
\mathrm{P}(r)={ }_{\text {loc }} \sum_{\alpha \in \mathrm{A}} \lambda_{\alpha}(r) u^{\alpha} \text { and } \mathrm{Q}(r)={ }_{\text {loc }} \sum_{\beta \in \mathrm{B}} \mu_{\beta}(r) v^{\beta} .
$$

So, the addition $\mathrm{P}+\mathrm{Q}$ writes locally:

$$
\mathrm{P}+\left.\mathrm{Q}\right|_{\text {loc }}: r \mapsto \sum_{\alpha \in \mathrm{A}} \lambda_{\alpha}(r) u^{\alpha}+\sum_{\beta \in \mathrm{B}} \mu_{\beta}(r) v^{\beta}=\sum_{\sigma \in \mathrm{C}} \nu_{\sigma}(r) w^{\sigma}
$$

where $\mathrm{C}$ is just the sum of the two sets of indices $\mathrm{A}$ and $\mathrm{B}$, and the family $\left(\nu_{\sigma}, w^{\sigma}\right)_{\sigma \in \mathrm{C}}$

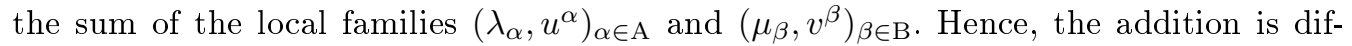
ferentiable. On the other hand, the multiplication by a scalar being differentiable in $\mathbf{K}$, the multiplication by a scalar in $\mathrm{E}$ is also differentiable. Therefore, this diffeology is a diffeology of vector space.

It is clear, by the very definition of generating families (art. 3.1), that the parametrizations $\diamond$ are generated by the family $\odot$. Now, let us prove that this fine diffeology is finest than any diffeology of vector space defined on E. 
3) Fineness. Let us consider E, provided with any other diffeology $\mathcal{D}$ of vector space. For any smooth parametrization $\lambda$ of $\mathbf{K}$ and any vector $u \in \mathrm{E}$, the parametrization $r \mapsto \lambda(r) u$ is differentiable, by differentiability of the multiplication by a scalar. Now, by differentiability of the addition, for any finite local family $\left(\lambda_{\alpha}, u^{\alpha}\right)_{\alpha \in \mathrm{A}}$, the parametrization $r \mapsto \sum_{\alpha \in \mathrm{A}} \lambda_{\alpha}(r) u^{\alpha}$ is differentiable, that is a plot of the diffeology $\mathcal{D}$. Then, the diffeology $\mathcal{D}$ if coarser than the fine diffeology defined above. Hence, the fine diffeology is the finest diffeology of vector space on $\mathrm{E}$.

13.2. Generating the fine diffeology. Let $\mathrm{E}$ be a vector space on $\mathbf{K}$ and $\mathrm{L}\left(\mathbf{K}^{n}, \mathrm{E}\right)$ be the set of all linear maps from $\mathbf{K}^{m}$ into E. Let $\mathrm{L}^{\star}\left(\mathbf{K}^{n}, \mathrm{E}\right)$ be the set of all injective maps from $\mathbf{K}^{m}$ into $\mathrm{E}$,

$$
\mathrm{L}^{\star}\left(\mathbf{K}^{m}, \mathrm{E}\right)=\left\{j \in \mathrm{L}\left(\mathbf{K}^{m}, \mathrm{E}\right) \mid \operatorname{ker}(j)=\{0\}\right\} .
$$

The two families

$$
\mathcal{F}=\bigcup_{m \in \mathbf{N}} \mathrm{L}\left(\mathbf{K}^{m}, \mathrm{E}\right) \quad \text { and } \quad \mathcal{F}^{\star}=\bigcup_{m \in \mathbf{N}} \mathrm{L}^{\star}\left(\mathbf{K}^{m}, \mathrm{E}\right),
$$

generate both the fine diffeology of $\mathrm{E}$.

Note that a parametrization $\mathrm{P}: \mathrm{U} \longrightarrow \mathrm{E}$ is a plot for the diffeology generated by $\mathcal{F}$ if and only if, for any $r_{0}$ in $\mathrm{U}$, there exists a superset $\mathrm{V}$ of $r_{0}$ in $\mathrm{U}$, an integer $m$, a smooth parametrization $\phi: \mathrm{V} \rightarrow \mathbf{K}^{m}$, a linear map $j: \mathbf{K}^{m} \rightarrow \mathrm{E}$, such that $\mathrm{P} \uparrow \mathrm{V}=j \circ \phi$. In other words, locally, $\mathrm{P}$ takes its values in a constant finite dimensional subspace $\mathrm{F} \subset \mathrm{E}$ such that the the coordinates of $\mathrm{P}$ for some basis of $\mathrm{F}$ are smooth. For the plots generated by $\mathcal{F}^{\star}, j$ is injective.

Proof. Let us prove that $\mathcal{F}$, as well as $\mathcal{F}^{\star}$, generate the fine diffeology.

Let us $\mathrm{P}: \mathrm{U} \rightarrow \mathrm{E}$ be a plot of the diffeology generated by $\mathcal{F}$ or by $\mathcal{F}^{\star}$. Pick a point $r_{0}$ in $\mathrm{U}$. By definition there exists a superset $\mathrm{V}$ of $r_{0}$, an integer $m$, a smooth parametrization $\phi: \mathrm{V} \longrightarrow \mathbf{K}^{m}$, a linear map $j: \mathbf{K}^{m} \rightarrow \mathrm{E}$, such that $\mathrm{P} \uparrow \mathrm{V}=j \circ \phi$. So, for any $r$ in $\mathrm{V}, \phi(r)=\sum_{k=1}^{m} \phi_{k}(r) \mathbf{e}_{k}$, where $\left(\mathbf{e}_{1}, \ldots, \mathbf{e}_{m}\right)$ is the canonical basis of $\mathbf{K}^{m}$, and $\phi_{k} \in \mathrm{C}^{\infty}(\mathrm{V}, \mathbf{K})$. Now, $\mathrm{P}(r)=j\left(\sum_{k=1}^{m} \phi_{k}(r) \mathbf{e}_{k}\right)=\sum_{k=1}^{m} \phi_{k}(r) j\left(\mathbf{e}_{k}\right)=\sum_{k=1}^{m} \phi_{k}(r) \mathbf{f}_{k}$ where $\mathbf{f}_{k}=j\left(\mathbf{e}_{k}\right)$. Therefore, $\mathrm{P}$ is a plot of the fine diffeology of $\mathrm{E}$, and $\left(\phi_{k}, \mathbf{f}_{k}\right)_{k=1}^{m}$ is a local family of the plot P. Note that $j$ can be chosen injective.

Conversely, let $\mathrm{P}: \mathrm{U} \rightarrow \mathrm{E}$ be a plot of the fine diffeology. Let $r_{0}$ be a point of $\mathrm{U}$. There exists an open superset $\mathrm{V}$ of $r_{0}$ in $\mathrm{U}$, an integer $\mathrm{N}$, a local family $\left(\lambda_{\alpha}, v^{\alpha}\right)_{\alpha=1}^{\mathrm{N}}$, with $\lambda_{\alpha} \in \mathrm{e}^{\infty}(\mathrm{V}, \mathbf{K}), v^{\alpha} \in \mathrm{E}$, and such that $\mathrm{P} \uparrow \mathrm{V}=\sum_{\alpha=1}^{\mathrm{N}} \lambda_{\alpha}(r) v^{\alpha}$. Let $\mathrm{F}$ be the vector space generated by the $v^{\alpha}$, and let $\mathbf{f}=\left(\mathbf{f}_{1}, \ldots, \mathbf{f}_{m}\right)$ be a basis of F. Let us decompose the vectors $v^{\alpha}$ on the basis $\mathbf{f}, v^{\alpha}=\sum_{k=1}^{m} v_{k}^{\alpha} \mathbf{f}_{k}$. Now, $\mathrm{P} \uparrow \mathrm{V}=\sum_{\alpha=1}^{\mathrm{N}} \sum_{k=1}^{m} \lambda_{\alpha}(r) v_{k}^{\alpha} \mathbf{f}_{k}=$ $\sum_{k=1}^{m} \phi_{k}(r) \mathbf{f}_{k}$, where $\phi_{k}(r)=\sum_{\alpha=1}^{\mathrm{N}} \lambda_{\alpha}(r) v_{k}^{\alpha}$. The $\phi_{k}$ are smooth maps defined on $\mathrm{V}$ with values in $\mathbf{K}$. Now, let $j: \mathbf{K}^{m} \longrightarrow \mathrm{E}$ be the linear map defined by $j\left(\mathbf{e}_{k}\right)=\mathbf{f}_{k}$ and $\phi: \mathrm{V}$ $\longrightarrow \mathbf{K}^{m}$ defined by $\phi=\left(\phi_{1}, \ldots, \phi_{m}\right)$. So, $\mathrm{P} \uparrow \mathrm{V}=j \circ \phi$, where $j$ is an injective linear map from $\mathbf{K}^{m}$ to $\mathrm{E}$ and $\phi$ belongs to $\mathrm{C}^{\infty}\left(\mathrm{V}, \mathbf{K}^{m}\right)$. Therefore, $\mathrm{P}$ is a plot of the diffeology generated by $\mathcal{F}^{\star}$, a fortiori by $\mathcal{F}$. Hence, the fine diffeology of $\mathrm{E}$ is generated by the set of linear maps, or injective linear maps, from $\mathbf{K}^{m}$ into $\mathrm{E}$, when $m$ runs over the integers.

13.3. Linear maps and fine diffeology. Let $\mathrm{E}$ and $\mathrm{F}$ be two diffeological vector spaces over K. Let $\mathrm{E}$ be equipped with the fine diffeology. Any linear map from $\mathrm{E}$ to $\mathrm{F}$ is differentiable. In other words, if $\mathrm{E}$ is fine, $\mathrm{LC}{ }^{\infty}(\mathrm{E}, \mathrm{F})=\mathrm{L}(\mathrm{E}, \mathrm{F})$. 
Proof. Let $(\mathrm{P} \uparrow \mathrm{V})(r)=\sum_{\alpha=1}^{\mathrm{N}} \lambda_{\alpha}(r) v_{\alpha}$ be a local expression of some plot $\mathrm{P}$ of E. Let $\mathrm{A} \in \mathrm{L}(\mathrm{E}, \mathrm{F})$, then we have $(\mathrm{A} \circ \mathrm{P} \uparrow \mathrm{V})(r)=\sum_{\alpha=1}^{\mathrm{N}} \lambda_{\alpha}(r) \mathrm{A}\left(v_{\alpha}\right)$. Since $\mathrm{A}\left(v_{\alpha}\right) \in \mathrm{F}$ for each $\alpha, \mathrm{P}$ is a plot of the fine diffeology of $\mathrm{F}$, therefore a plot of any vector space diffeology. Thus, $\mathrm{A}$ is differentiable, and $\mathrm{L}(\mathrm{E}, \mathrm{F}) \subset \mathrm{LC}^{\infty}(\mathrm{E}, \mathrm{F})$. The converse inclusion is clear.

13.4. The fine linear category. Thanks to (art. 13.3) the fine diffeological spaces define a subcategory of the linear diffeological category (art. 12.5), we shall call it the fine linear category. Objects of this category are all vector spaces. And, according to the above proposition, morphisms of this category are just linear maps. Hence, the fine linear category coincides with the usual linear category over the chosen field. In other words, the functor from the linear category to the fine linear category, which associates to each vector space the same space equipped with the fine diffeology, is a full faithful functor [McL71].

13.5. Subspaces of fine diffeological vector spaces. Let $\mathrm{E}$ be a vector space over $\mathbf{K}$, equipped with its fine diffeology. Let $\mathrm{F} \subset \mathrm{E}$ be any vector subspace. The subset diffeology of $\mathrm{F}$, inherited from $\mathrm{E}$, is the fine diffeology. In other words, the injection of $\mathrm{F}$ into $\mathrm{E}$ where $\mathrm{E}$ and $\mathrm{F}$ are equipped with their fine diffeology is an induction.

Proof. The injection is linear, so it is differentiable (art. 13.3). Let us check now that if a plot $\mathrm{P}: \mathrm{U} \rightarrow \mathrm{E}$ takes its values in $\mathrm{F}$, then it is a plot for the fine diffeology of $\mathrm{F}$. For all $r_{0} \in \mathrm{U}$ there exists a superset $\mathrm{V}$ of $r_{0}$, an injection $j: \mathbf{K}^{m} \rightarrow \mathrm{E}$ and a smooth parametrization $\phi: \mathrm{V} \rightarrow \mathbf{K}^{m}$ such that $\mathrm{P} \uparrow \mathrm{V}=j \circ \phi$. Since $\mathrm{P}$ takes its values in $\mathrm{F}$ so does $j \circ \phi$. Let $\mathrm{H}=\operatorname{span}(\operatorname{val}(\phi))$ be the subspace of $\mathbf{K}^{m}$ generated by $\operatorname{val}(\phi)$ and $j^{\prime}=j \uparrow \mathrm{H}$. Thus, we have $\mathrm{P} \uparrow \mathrm{V}=j^{\prime} \circ \phi$ where $j^{\prime}$ is a linear injection from $\mathrm{H}$ to $\mathrm{F}$. Therefore, $\mathrm{P}$ is a plot of the fine diffeology of $\mathrm{F}$.

13.6. Products and quotients of fine vector spaces. The product of any finite family of fine vector spaces is a fine vector space for the product diffeology. As well, the quotient of a fine vector space by a subspace is a fine vector space for the quotient diffeology.

Proof. Let $\mathrm{E}$ and $\mathrm{E}^{\prime}$ be two fine diffeological vector spaces. Let $\mathrm{Q}: \mathrm{U} \longrightarrow \mathrm{E} \times \mathrm{E}^{\prime}$ be a plot, that is $\mathrm{Q}=\mathrm{P} \times \mathrm{P}^{\prime}$ where $\mathrm{P}: \mathrm{U} \rightarrow \mathrm{E}$ and $\mathrm{P}^{\prime}: \mathrm{U} \rightarrow \mathrm{E}^{\prime}$ are two plots (art. 5.7). Now, let $r_{0} \in \mathrm{U}$, there exists two supersets $\mathrm{V}$ and $\mathrm{V}^{\prime}$ of $r_{0}$, two local families defined on $\mathrm{V}$, $\left(\lambda_{\alpha}, \mathrm{X}_{\alpha}\right)_{\alpha \in \mathrm{A}}$ and $\left(\lambda_{\alpha^{\prime}}^{\prime}, \mathrm{X}_{\alpha^{\prime}}^{\prime}\right)_{\alpha^{\prime} \in \mathrm{A}^{\prime}}$, such that $\mathrm{P} \uparrow \mathrm{V}: r \mapsto \sum_{\alpha} \lambda_{\alpha}(r) \mathrm{X}_{\alpha}$ and $\mathrm{P}^{\prime} \uparrow \mathrm{V}^{\prime}: r \mapsto$ $\sum_{\alpha^{\prime}} \lambda_{\alpha^{\prime}}(r) \mathrm{X}_{\alpha^{\prime}}$. Let's define $\mathrm{V}^{\prime \prime}=\mathrm{V} \cap \mathrm{V}^{\prime}$, we get:

$$
\begin{aligned}
\left(\mathrm{Q} \uparrow \mathrm{V}^{\prime \prime}\right)(r) & =\left(\sum_{\alpha \in \mathrm{A}} \lambda_{\alpha}(r) \mathrm{X}_{\alpha}, \sum_{\alpha^{\prime} \in \mathrm{A}^{\prime}} \lambda_{\alpha^{\prime}}(r) \mathrm{X}_{\alpha^{\prime}}^{\prime}\right) \\
& =\left(\sum_{\alpha \in \mathrm{A}} \lambda_{\alpha}(r) \mathrm{X}_{\alpha}, 0\right)+\left(0, \sum_{\alpha^{\prime} \in \mathrm{A}^{\prime}} \lambda_{\alpha^{\prime}}(r) \mathrm{X}_{\alpha^{\prime}}^{\prime}\right) \\
& =\sum_{\alpha \in \mathrm{A}} \lambda_{\alpha}(r)\left(\mathrm{X}_{\alpha}, 0\right)+\sum_{\alpha^{\prime} \in \mathrm{A}^{\prime}} \lambda_{\alpha^{\prime}}(r)\left(0, \mathrm{X}_{\alpha^{\prime}}^{\prime}\right) .
\end{aligned}
$$

This exhibits the disjoint union of the two families $\left(\lambda_{\alpha}, \mathrm{X}_{\alpha}\right)_{\alpha \in \mathrm{A}}$ and $\left(\lambda_{\alpha^{\prime}}^{\prime}, \mathrm{X}_{\alpha^{\prime}}^{\prime}\right)_{\alpha^{\prime} \in \mathrm{A}^{\prime}}$ over $\mathrm{V}^{\prime \prime}$ as a local family of $\mathrm{Q} \uparrow \mathrm{V}^{\prime \prime}$. Therefore $\mathrm{Q}$ is a plot of the fine diffeology of $\mathrm{E} \times \mathrm{E}^{\prime}$. The extension to any finite number of factors is immediate. 
13.7. The functional diffeology of $\mathrm{L}\left(\mathrm{E}, \mathrm{E}^{\prime}\right)$. Let $\mathrm{E}$ and $\mathrm{E}^{\prime}$ be two fine vector spaces over K. The functional diffeology of the space of linear maps $\mathrm{Le}^{\infty}\left(\mathrm{E}, \mathrm{E}^{\prime}\right)=\mathrm{L}\left(\mathrm{E}, \mathrm{E}^{\prime}\right)$ is characterized as follows.

A parametrization $\mathrm{P}: \mathrm{U} \rightarrow \mathrm{L}\left(\mathrm{E}, \mathrm{E}^{\prime}\right)$ is a plot of the functional diffeology if for any $r_{0} \in \mathrm{U}$ and for any vector subspace $\mathrm{F} \subset \mathrm{E}$ of finite dimension, there exists an open superset $\mathrm{V}$ of $r_{0}$, and a vector subspace of finite dimension $\mathrm{F}^{\prime} \subset \mathrm{E}^{\prime}$ such that:

1. For any $r \in \mathrm{V}$, the linear map $\mathrm{P}(r) \uparrow \mathrm{V}$ belongs to $\mathrm{L}\left(\mathrm{F}, \mathrm{F}^{\prime}\right)$.

2. The parametrization $r \mapsto \mathrm{P}(r) \uparrow \mathrm{F}$, restricted to $\mathrm{V}$, is a plot of $\mathrm{L}\left(\mathrm{F}, \mathrm{F}^{\prime}\right)$.

Note that, for the second condition, $r \mapsto \mathrm{P}(r) \uparrow \mathrm{F}$ is a parametrization of a space of finite dimensional linear maps, or matrices. The condition to be a plot is just that each coefficient of the matrix is a smooth function.

Proof. Let $\mathrm{P}: \mathrm{U} \rightarrow \mathrm{L}\left(\mathrm{E}, \mathrm{E}^{\prime}\right)$ be a plot of the functional diffeology. Let us show that it satisfies the condition of the proposition. Let $\mathrm{F} \subset \mathrm{E}$ be any vector subspace of finite dimension. Let $\left(u_{1}, \ldots, u_{m}\right)$ be a basis of $\mathrm{F}$. Let $r_{0} \in \mathrm{U}$, by definition of the functional diffeology, for any $k=1 \ldots m$, the map $r \mapsto \mathrm{P}(r)\left(u_{k}\right)$ is a plot of $\mathrm{E}^{\prime}$, so there exists a superset $\mathrm{V}_{k}$ of $r_{0}$, a finite set of indices $\mathrm{A}_{k}$, a family $\left(\lambda_{k, \alpha}\right)_{\alpha \in \mathrm{A}_{k}}$ of smooth parametrizations of $\mathbf{K}$, a family $\left(w_{k, \alpha}\right)_{\alpha \in \mathrm{A}_{k}}$ of vectors of $\mathrm{E}$, such that, for any $r \in \mathrm{V}_{k}$ :

$$
\mathrm{P}(r)\left(u_{k}\right)=\sum_{\alpha \in \mathrm{A}_{k}} \lambda_{k, \alpha}(r) w_{k, \alpha}
$$

For all $\left(c_{1}, \ldots, c_{m}\right) \in \mathbf{K}^{m}$, for all $r \in \mathrm{V}=\cap_{k=1}^{m} \mathrm{~V}_{k}$ we have:

$$
\mathrm{P}(r)\left(\sum_{k=1}^{m} c_{k} u_{k}\right)=\sum_{k=1}^{m} c_{k} \mathrm{P}(r)\left(u_{k}\right)=\sum_{k=1}^{m} \sum_{\alpha \in \mathrm{A}_{k}} c_{k} \lambda_{k, \alpha}(r) w_{k, \alpha} .
$$

Let $\mathrm{F}^{\prime}$ be the subspace of $\mathrm{E}^{\prime}$ spanned by the vectors $\cup_{k=1}^{m}\left\{w_{k, \alpha}\right\}_{\alpha \in \mathrm{A}_{k}}$, for any $u \in \mathrm{F}$, $\mathrm{P}(r)(u) \in \mathrm{F}^{\prime}$. The first condition above is checked. Now, let $\left(v_{1}, \ldots, v_{n}\right)$ be a basis of $\mathrm{F}^{\prime}$, such that for any $k=1 \ldots m$ and any $\alpha \in \mathrm{A}_{k}, w_{k, \alpha}=\sum_{j=1}^{n} w_{k, \alpha}^{j} v_{j}$. Replacing $w_{k, \alpha}$ by this expression we get:

$$
\mathrm{P}(r)\left(\sum_{k=1}^{m} c_{k} u_{k}\right)=\sum_{k=1}^{m} \sum_{\alpha \in \mathrm{A}_{k}} c_{k} \lambda_{k, \alpha}(r) \sum_{j=1}^{n} w_{k, \alpha}^{j} v_{j}=\sum_{j=1}^{n}\left(\sum_{k=1}^{m} \sum_{\alpha \in \mathrm{A}_{k}} c_{k} \lambda_{k, \alpha}(r) w_{k, \alpha}^{j}\right) v_{j} .
$$

Hence, defining

$$
\phi_{j}(r)=\sum_{k=1}^{m} \sum_{\alpha \in \mathrm{A}_{k}} c_{k} \lambda_{k, \alpha}(r) w_{k, \alpha}^{j},
$$

we get a family of smooth parametrizations $\left(\phi_{j}\right)_{j=1}^{n}$ of $\mathbf{K}$ such that, for any $r \in \mathrm{V}$ :

$$
\mathrm{P}(r)(u)=\sum_{j=1}^{n} \phi_{j}(r) v_{j}
$$

The expression of $\mathrm{P}(r)$ above clearly shows that $r \mapsto \mathrm{P}(r) \uparrow \mathrm{F}$ is a plot of the functional diffeology of $\mathrm{L}\left(\mathrm{F}, \mathrm{F}^{\prime}\right)$. Indeed, by choosing for $u$ successively each vector of a basis of $\mathrm{F}$, the last expression shows that the components of $\mathrm{P}(r) \uparrow \mathrm{F}$ are smooth parametrizations of $\mathbf{K}$, which is the condition, in finite dimension, to be a plot of the functional diffeology. Hence, we proved the first part of the proposition above. 
Conversely, let us assume that the parametrization $\mathrm{P}$ satisfies the condition of the proposition, and let us show that $\mathrm{P}$ is a plot of the functional diffeology. Let us consider a plot $\mathrm{Q}: \mathrm{V} \rightarrow \mathrm{E}$. By definition, for any $s_{0} \in \mathrm{V}$, there exists a superset $\mathrm{W}$ of $s_{0}$, a finite set of indices A, a family $\left(\lambda_{\alpha}\right)_{\alpha \in \mathrm{A}}$ of smooth parametrizations of $\mathbf{K}$, a family $\left(v_{\alpha}\right)_{\alpha \in \mathrm{A}}$ of vectors of $\mathrm{E}$ such that for any $s \in \mathrm{V}, \mathrm{Q}(s)=\sum_{\alpha \in \mathrm{A}} \lambda_{\alpha}(s) v_{\alpha}$. Let $\mathrm{F} \subset \mathrm{E}$ be the vector subspace spanned by the vectors $v_{\alpha}$. Hence, for any $r_{0} \in \mathrm{U}$, there exists an open superset $\mathrm{U}^{\prime}$ of $r_{0}$ and a vector subspace $\mathrm{F}^{\prime} \subset \mathrm{E}^{\prime}$ such that $\mathrm{P}(r)(\mathrm{F}) \subset \mathrm{F}^{\prime}$ for any $r \in \mathrm{U}^{\prime}$. Thus, for any $(r, s) \in \mathrm{U}^{\prime} \times \mathrm{W}, \mathrm{P}(r)(\mathrm{Q}(s))=\mathrm{P}(r)\left(\sum_{\alpha \in \mathrm{A}} \lambda_{\alpha}(s) v_{\alpha}\right)=\sum_{\alpha \in \mathrm{A}} \lambda_{\alpha}(s) \mathrm{P}(r)\left(v_{\alpha}\right) \in \mathrm{F}^{\prime}$. And since the parametrization $r \mapsto \mathrm{P}(r) \uparrow \mathrm{F}$ is a plot of the functional diffeology, the parametrization $\mathrm{P} \cdot \mathrm{Q}:(r, s) \mapsto \mathrm{P}(r)(\mathrm{Q}(s))$ is a smooth parametrization of $\mathrm{F}^{\prime} \subset \mathrm{E}^{\prime}$, thus $\mathrm{P} \cdot \mathrm{Q}$ is a smooth parametrization of $\mathrm{E}^{\prime}$, because any finite subspace is embedded in $\mathrm{E}^{\prime}$. Therefore, $\mathrm{P}$ is a plot of the functional diffeology of $\mathrm{L}\left(\mathrm{E}, \mathrm{E}^{\prime}\right)$. This completes the proof of the proposition.

13.8. The fine topology. The $\mathrm{D}$-topology of a fine diffeological vector space $\mathrm{E}$ has a simple characterization. A part $\Omega \subset \mathrm{E}$ is $\mathrm{D}$-open if and only if its intersection with any finite dimensional vector space $\mathrm{F} \subset \mathrm{E}$ is open in $\mathrm{F}$. Indeed, the diffeology of $\mathrm{E}$ is generated by the the linear injections $j: \mathbf{K}^{n} \rightarrow \mathrm{E}$ (art. 13.2), where $n$ runs over $\mathbf{N}$, hence $\Omega$ is D-open if and only if its inverse image by every one of these injections is open in $\mathbf{K}^{n}$. Or, equivalently, if the intersection of $\Omega$ with any vector subspace $\mathrm{F}$, of finite dimension, is open for the smooth topology of $\mathrm{F}$. We recognize here the so-called finite topology of functional analysis [Tyc35].

13.9. Standard finite dimensional vector spaces. The standard diffeology on $\mathbf{K}^{n}$ is the fine diffeology. Indeed, any plot $\mathrm{P}: \mathrm{U} \longrightarrow \mathbf{K}^{n}$ decomposes over the standard basis $\left(\mathbf{e}_{i}\right)_{i=1}^{n}$, that is $\mathrm{P}: r \mapsto \sum_{i=1}^{n} \mathrm{P}_{i}(r) \mathbf{e}_{i}$, where the $\mathrm{P}_{i}$ are smooth parametrizations of the field $\mathbf{K}$.

14. Euclidean and hermitian diffeological vector spaces. The definition of euclidean or hermitian structures on diffeological vector spaces is a natural extension of the standard definitions. They will be applied in the following in the study of infinite spheres and infinite projective spaces.

14.1. Euclidean and hermitian diffeological structures. Let $\mathrm{E}$ be a real (respectively complex) diffeological vector space, and $(\mathrm{X}, \mathrm{Y}) \mapsto \mathrm{X} \cdot \mathrm{Y}$ be an euclidean (respectively hermitian) product defined on E. If the euclidean (respectively hermitian) product is differentiable, $(\mathrm{E}, \cdot)$ is called a euclidean (respectively hermitian) diffeological vector space.

14.2. Fine euclidean or hermitian spaces. Any real (respectively complex) diffeological vector space E, equipped with its fine diffeology and equipped with any euclidean (respectively hermitian) structure is an euclidean (respectively hermitian) diffeological vector space.

Proof. This is a consequence of the property of linear maps to be differentiable on fine diffeological vector spaces (art. 13.3).

14.3. Uniqueness for finite dimensional euclidean spaces. The diffeology of any finite dimensional euclidean (or hermitian) diffeological space is the fine diffeology. 
Proof. Let $\left(\mathbf{e}_{1}, \ldots, \mathbf{e}_{n}\right)$ be an orthonormal basis of E. Let $\mathrm{P}: \mathrm{U} \longrightarrow \mathrm{E}$ be a plot of E. For any $r$ in $\mathrm{U}, \mathrm{P}(r)=\sum_{k=1}^{n}\left(\mathbf{e}_{k} \cdot \mathrm{P}(r)\right) \mathbf{e}_{k}$. Each map $\mathrm{P}_{k}(r): r \mapsto \mathbf{e}_{k} \cdot \mathrm{P}(r)$ is differentiable by hypothesis. Hence, $\mathrm{P}$ is a plot of the fine diffeology.

14.4. D-topology and topology of the norm. The norm topology of a hermitian diffeological space E does not necessarily coincide with the D-topology. But, any open ball $\mathrm{B}(x, \rho)$, centered in $x \in \mathrm{E}$, of radius $\rho$, is $\mathrm{D}$-open. Indeed, its preimage by any plot $\mathrm{P}: \mathrm{U} \rightarrow \mathrm{E}$ is the preimage of ] $-\infty, \rho^{2}\left[\right.$ by $r \mapsto\|x-\mathrm{P}(r)\|^{2}$, but this map is differentiable hence $\mathrm{D}$-continuous. Thus, the ball $\mathrm{B}(x, \rho)$ is $\mathrm{D}$-open. We can deduce, using the differentiability of translation and homotheties, that any open set for the norm topology is D-open. In other words, the topology of the norm is weaker than the D-topology.

15. Diffeological manifolds. Using diffeological vector spaces, we extend the ordinary definition of manifolds to manifolds modeled on diffeological vector spaces. Note that to be a manifold is not an extra structure added to the diffeology, but it is a property of the diffeology. Once a set is equipped with a diffeology, this space is or is not a manifold, it depends only on the diffeology. Hence diffeologies are or are not manifold diffeologies.

15.1. Manifolds. Let $\mathrm{X}$ be a diffeological space, and let $\mathrm{E}$ be a diffeological vector space. We say that $\mathrm{X}$ is a diffeological manifold modeled on $\mathrm{E}$ if $\mathrm{X}$ is locally diffeomorphic to $\mathrm{E}$ at each point. In other words, if for any $x \in \mathrm{X}$ there exists a local diffeomorphism (art. 6.8) $\mathrm{F}: \mathrm{U} \rightarrow \mathrm{X}$, called chart, such that $\mathrm{U} \subset \mathrm{E}$ and $x \in \mathrm{F}(\mathrm{U})$.

15.2. Generating manifolds. Let $\mathrm{E}$ be some diffeological vector space. A diffeological space $\mathrm{X}$ is a diffeological manifold modeled on $\mathrm{E}$ if and only if there exists a family $\mathcal{A}$ of local diffeomorphisms from $\mathrm{E}$ to $\mathrm{X}$, called charts, generating the diffeology of $\mathrm{X}$. In other words, for a diffeological space, to be or not to be a manifold (modeled on some diffeological vector space) is a property not an extra structure.

Any family of charts generating the diffeology of $\mathrm{X}$ is called an atlas of $\mathrm{X}$. Note that there exists an atlas made up with all the local diffeomorphisms from $\mathrm{E}$ to $\mathrm{X}$, this atlas is called the saturated, or maximal, atlas of $\mathrm{X}$.

Proof. Let $\mathrm{X}$ be generated by a family $\mathcal{A}$ of local diffeomorphisms from $\mathrm{E}$ to $\mathrm{X}$. Pick any point $x \in \mathrm{X}$, and let $\mathrm{P}:\{0\} \rightarrow \mathrm{X}$ be the constant plot such that $\mathrm{P}(0)=x$. By hypothesis, there exists a chart $\mathrm{F} \in \mathcal{A}$ and a lifting $\mathrm{Q}:\{0\} \rightarrow \mathrm{E}$ such that $\mathrm{P}=\mathrm{F} \circ \mathrm{Q}$. Hence, $\mathrm{F}$ is a local diffeomorphism from $\mathrm{E}$ to $\mathrm{X}$, such that $x \in \operatorname{val}(\mathrm{F})$. Therefore $\mathrm{X}$ is a diffeological manifold modeled on E. Conversely, let us assume that $\mathrm{X}$ is a diffeological manifold, that is locally diffeomorphic to $\mathrm{E}$ at each point. Then, let us choose for each point $x \in \mathrm{X}$, a local diffeomorphism $\phi: \mathrm{U} \rightarrow \mathrm{X}$ such that $x \in \phi(\mathrm{U})$. Let $\mathcal{A}$ be the set of all these chosen local diffeomorphisms, when $x$ runs over $\mathrm{X}$. Let $\mathrm{P}: \mathrm{V} \rightarrow \mathrm{X}$ be a plot and $r \in \mathrm{V}$, let $x=\mathrm{P}(r)$ and $\phi \in \mathcal{A}$ such that $x \in \phi(\mathrm{U})$. Now, let $\mathrm{Q}=\phi^{-1} \circ \mathrm{P} \uparrow \mathrm{W}$, where $\mathrm{W}=\mathrm{P}^{-1}(\phi(\mathrm{U}))$. Since $\phi$ is a local diffeomorphism $\phi(\mathrm{U})$ is $\mathrm{D}$-open. And since $\mathrm{P}$ is $\mathrm{D}$-continuous, $\mathrm{W}$ is open. Hence, $\mathrm{Q}$ is a local lifting of $\mathrm{P}$ along $\phi$. Therefore, any plot of $\mathrm{X}$ can be lifted along some element of the family $\mathcal{A}$. And, this is the definition of a generating family of $\mathrm{X}$. Thus, the diffeology of $\mathrm{X}$ is generated by a family of local diffeomorphisms from $\mathrm{E}$ to $\mathrm{X}$. 
15.3. Standard manifolds. The diffeological manifolds modeled on the numerical space $\mathbf{R}^{n}$, for some integer $n$, are the standard manifolds of dimension $n$. Note that the standard manifolds form a full faithful subcategory [McL71] of the category \{Diffeology\}.

15.4. Submanifolds. Let $\mathrm{X}$ be a diffeological space and $\mathrm{M} \subset \mathrm{X}$. The subset $\mathrm{M}$ will be called a submanifold of $\mathrm{X}$ if, equipped with the subset diffeology (art. 4.6), $\mathrm{M}$ is a manifold. Subsets of X are just subspaces or are submanifolds, depending on the induced diffeology. Note that submanifolds can be embedded or not. For example, an irrational winding in the 2-torus is just a submanifold, diffeomorphic to $\mathbf{R}$, while a rational winding is an embedded submanifold, diffeomorphic to $\mathrm{S}^{1}$. Another example, the infinite sphere $\mathcal{S}$ defined below is an embedded submanifold of the Hilbert space $\mathcal{H}$ (art. 17.2) .

\section{THE DIFFEOLOGICAL INFINITE HOPF FIBRATION}

In this chapter we introduce the unit sphere $\mathcal{S}$ of the Hilbert space $\mathcal{H}$ of square-summable sequences. The sphere $\mathcal{S}$ is equipped with the subset diffeology of the fine diffeology of $\mathcal{H}$. We call it the infinite sphere. We show that the infinite sphere is a contractible diffeological manifold modeled on $\mathcal{H}$. We construct then the infinite projective space $\mathcal{P}$, which is also the quotient of the infinite sphere by the action of $\mathcal{S}^{1}$. This defines the infinite Hopf fibration. We show, in particular, that $\mathcal{P}$, equipped with the quotient diffeology of $\mathcal{S}$, is a diffeological manifold modeled on $\mathcal{H}$.

16. The space of square-summable sequences. Here, we describe the main construction relative to the space of complex square-summable sequences, equipped with the fine diffeology.

16.1. The fine hermitian space $\mathcal{H}$. Let $\mathcal{H}$ be the set of square-sumable complex sequences, indexed by the nonzero integers.

$$
\mathcal{H}=\left\{\mathrm{Z}: \mathbf{N}^{\star} \rightarrow \mathbf{C} \mid \mathrm{Z}=\left(\mathrm{Z}_{k}\right)_{k=1}^{\infty}, \sum_{k=1}^{\infty} \mathrm{Z}_{k}^{*} \mathrm{Z}_{k}=\lim _{N \rightarrow \infty} \sum_{k=1}^{N} \mathrm{Z}_{k}^{*} \mathrm{Z}_{k}<\infty\right\},
$$

where $\mathbf{N}^{\star}$ denotes the set of nonzero positive integers and $z^{*}$ denotes the complex conjugate of a complex number $z$. The space of complex numbers $\mathbf{C}$ is naturally equipped with the standard diffeology. And in the following, the space $\mathcal{H}$ is equipped with the fine diffeology (art. 13.1).

Let us recall that a parametrization $\mathrm{P}: \mathrm{U} \rightarrow \mathcal{H}$ is a plot for the fine diffeology if and only if, for each $r_{0}$ in $\mathrm{U}$ there exists an open superset $\mathrm{V}$ of $r_{0}$ in $\mathrm{U}$, and a local family $\left(\lambda_{\alpha}, \mathrm{Z}_{\alpha}\right)_{\alpha \in \mathrm{A}}, \# \mathrm{~A}<\infty$, such that:

$$
(\mathrm{P} \uparrow \mathrm{V})(r)=\sum_{\alpha \in \mathrm{A}} \lambda_{\alpha}(r) \mathrm{Z}_{\alpha}, \text { with } \lambda_{\alpha} \in \mathrm{C}^{\infty}(\mathrm{V}, \mathbf{C}) \text { and } \mathrm{Z}_{\alpha} \in \mathcal{H}
$$

Note that the functions $\lambda_{\alpha}$ are smooth for the real smooth structure of $\mathbf{C}$, we are not talking about holomorphic functions here.

Now let us recall the usual sesquilinear product defined on $\mathcal{H}$ :

$$
\text { For all } \mathrm{Z}, \mathrm{Z}^{\prime} \in \mathcal{H}, \quad \mathrm{Z} \cdot \mathrm{Z}^{\prime}=\sum_{k=1}^{\infty} \mathrm{Z}_{k}^{*} \mathrm{Z}_{k}^{\prime}
$$


The sesquilinear map $\left(\mathrm{Z}, \mathrm{Z}^{\prime}\right) \mapsto \mathrm{Z} \cdot \mathrm{Z}^{\prime}$ is a hermitian product. So, the pair $(\mathcal{H}, \cdot)$ is a fine hermitian diffeological vector space over $\mathbf{C}$ (art. 14.2). We will denote, as usual, by $\|\cdot\|$ the norm associated to the hermitian product:

$$
\text { For all } \mathrm{Z} \in \mathcal{H}, \quad\|\mathrm{Z}\|=\sqrt{\mathrm{Z} \cdot \mathrm{Z}} \text {. }
$$

And we introduce also the notation $\operatorname{pr}_{k}$ for the $k$-th projection from $\mathcal{H}$ onto $\mathbf{C}$

$$
\text { For all } \mathrm{Z}=\left(\mathrm{Z}_{k}\right)_{k=1}^{\infty} \in \mathcal{H}, \quad \operatorname{pr}_{k}(\mathrm{Z})=\mathrm{Z}_{k} \in \mathbf{C}, k>0 \text {. }
$$

17. The infinite sphere. The unit sphere $\mathcal{S}$ of the Hilbert space $\mathcal{H}$ is defined as usual by:

$$
\mathcal{S}=\left\{\left.\mathrm{Z} \in \mathcal{H}\left|\mathrm{Z} \cdot \mathrm{Z}=\sum_{k=1}^{\infty}\right| \mathrm{Z}_{k}\right|^{2}=1\right\} .
$$

The sphere $\mathcal{S}$ will be called the infinite sphere and will be equipped with the subset diffeology (art. 4.6) of the fine diffeology of $\mathcal{H}$.

17.1. The plots of the unit sphere. By definition (art. 4.6), a plot $\mathrm{P}: \mathrm{U} \longrightarrow \mathcal{S}$ is a plot of $\mathcal{H}$ taking its values in $\mathcal{S}$. That is, for any point $r_{0}$ of $\mathrm{U}$ there exists an open superset $\mathrm{V}$ of $r_{0}$ and a finite local family $\left(\lambda_{\alpha}, \mathrm{Z}_{\alpha}\right)_{\alpha \in \mathrm{A}}$, such that:

$$
\text { for all } r \in \mathrm{V}, \mathrm{P}(r)=\sum_{\alpha \in \mathrm{A}} \lambda_{\alpha}(r) \mathrm{Z}_{\alpha} \quad \text { with } \sum_{\alpha, \beta \in \mathrm{A}} \lambda_{\alpha}^{*}(r) \lambda_{\beta}(r) \mathrm{Z}_{\alpha} \cdot \mathrm{Z}_{\beta}=1,
$$

for some $\lambda_{\alpha} \in \mathfrak{C}^{\infty}(\mathrm{V}, \mathbf{C})$ and $\mathrm{Z}_{\alpha} \in \mathcal{H}$. For example, pick two orthonormal vectors $\mathrm{Z}_{a}$ and $\mathrm{Z}_{b}$ of $\mathcal{H}$, that is $\left\|\mathrm{Z}_{a}\right\|=\left\|\mathrm{Z}_{b}\right\|=1$ and $\mathrm{Z}_{a} \cdot \mathrm{Z}_{b}=0$. The parametrization $\mathrm{P}: t \mapsto$ $\cos (t) \mathrm{Z}_{a}+\sin (t) \mathrm{Z}_{b}$, where $t \in \mathbf{R}$, is a plot of $\mathcal{S}$.

17.2. The infinite sphere as a diffeological space. The infinite sphere $\mathcal{S}$, equipped with the subset diffeology of the fine diffeology of $\mathcal{H}$, is a manifold modeled on $\mathcal{H}$ (art. 15.1). Moreover, the sphere $\mathcal{S}$ is embedded in $\mathcal{H}$ (art. 6.6) and contractible (art. 8.4).

Proof. The proof will be given in several steps. First of all we shall identify the Hilbert space of complex summable sequences with the space of real summable sequences. This will identify, at the same time, the unit spheres of these two spaces. Then, we shall show that the real infinite sphere is a contractible manifold modeled on the Hilbert space of real summable sequences, and then that it is embedded, which will prove the proposition.

Let us first introduce the Hilbert space $\mathcal{H}_{\mathbf{R}}$ :

$$
\mathcal{H}_{\mathbf{R}}=\left\{\mathrm{X}: \mathbf{N}^{\star} \rightarrow \mathbf{R} \mid \mathrm{X}=\left(\mathrm{X}_{k}\right)_{k=1}^{\infty}, \sum_{k=1}^{\infty} \mathrm{X}_{k}^{2}=\lim _{N \longrightarrow \infty} \sum_{k=1}^{N} \mathrm{X}_{k}^{2}<\infty\right\}
$$

equipped with the fine diffeology. This space can be also considered as the subspace of $\mathcal{H}$ in which all coefficients are real.

STEP 1: The spaces $\mathcal{H}$ and $\mathcal{H}_{\mathbf{R}}$ are diffeomorphic. Let us introduce the following two maps, for any $\mathrm{X}=\left(\mathrm{X}_{k}\right)_{k=1}^{\infty}$ belonging to $\mathcal{H}_{\mathbf{R}}$, or for any $\mathrm{Z}=\left(\mathrm{Z}_{k}\right)_{k=1}^{\infty}$ belonging to $\mathcal{H}$ :

$$
\left\{\begin{array}{c}
\text { Fold }: \mathrm{X} \mapsto \mathrm{Z} \text { with } \mathrm{Z}_{k}=\mathrm{X}_{2 k-1}+i \mathrm{X}_{2 k} \text {, for all } k>0 \\
\text { Unfold }: \mathrm{Z} \mapsto \mathrm{X} \text { with } \mathrm{X}_{2 k+1}=\Re\left(\mathrm{Z}_{k+1}\right) \text { and } \mathrm{X}_{2 k}=\Im\left(\mathrm{Z}_{k}\right) \text {, for all } k \geq 0,
\end{array}\right.
$$


where $\Re$ and $\Im$ denote the real and imaginary parts. These two maps are bijective and each other's inverse: Unfold $=$ Fold $^{-1}$. Let us now check that they are differentiable. Let $\mathrm{P}: \mathrm{U} \rightarrow \mathcal{H}_{\mathbf{R}}$ be a plot. Locally $\mathrm{P}(r)$ writes $\sum_{\alpha \in \mathrm{A}} \lambda_{\alpha}(r) \mathrm{X}_{\alpha}$, where $\left(\lambda_{\alpha}, \mathrm{X}_{\alpha}\right)_{\alpha \in \mathrm{A}}$ is a local family. Now,

$$
\operatorname{Fold}(\mathrm{P}(r))=\operatorname{Fold}\left(\sum_{\alpha \in \mathrm{A}} \lambda_{\alpha}(r) \mathrm{X}_{\alpha}\right)=\sum_{\alpha \in \mathrm{A}} \lambda_{\alpha}(r) \operatorname{Fold}\left(\mathrm{X}_{\alpha}\right),
$$

since Fold is $\mathbf{R}$-linear and the $\lambda_{\alpha}$ are real valued functions. Writing $\mathrm{Z}_{\alpha}=\operatorname{Fold}\left(\mathrm{X}_{\alpha}\right)$, we have $\operatorname{Fold}(\mathrm{P}(r))=\sum_{\alpha \in \mathrm{A}} \lambda_{\alpha}(r) \mathrm{Z}_{\alpha}$, so Fold $\circ \mathrm{P}$ is a plot of $\mathcal{H}$. Therefore the map Fold is differentiable. Conversely, let us consider a plot $\mathrm{P}: \mathrm{U} \longrightarrow \mathcal{H}$. Locally $\mathrm{P}(r)$ writes $\sum_{\alpha \in \mathrm{A}} \lambda(r) \mathrm{Z}_{\alpha}$, where $\left(\lambda_{\alpha}, \mathrm{Z}_{\alpha}\right)_{\alpha \in \mathrm{A}}$ is a local family. But now the $\lambda_{\alpha}$ are complex valued functions and the $\mathrm{Z}_{\alpha}$ are complex vectors. Let us decompose $\lambda_{\alpha}=\mu_{\alpha}+i \nu_{\alpha}$, where $\mu_{\alpha}$ and $\nu_{\alpha}$ are real valued functions. Now, $\mathrm{P}(r)=\sum_{\alpha \in \mathrm{A}}\left[\mu_{\alpha}(r)+i \nu_{\alpha}(r)\right] \mathrm{Z}_{\alpha}=\sum_{\alpha \in \mathrm{A}} \mu_{\alpha}(r) \mathrm{Z}_{\alpha}+$ $\sum_{\alpha \in \mathrm{A}} \nu_{\alpha}(r) i \mathrm{Z}_{\alpha}$. But $i \mathrm{Z}_{\alpha}$ is still an element of $\mathcal{H}$. Hence, the union of the two local families $\left(\mu_{\alpha}, \mathrm{Z}_{\alpha}\right)_{\alpha \in \mathrm{A}}$ and $\left(\nu_{\alpha}, i \mathrm{Z}_{\alpha}\right)_{\alpha \in \mathrm{A}}$ is still a local family $\left(\rho_{\beta}, \zeta_{\beta}\right)_{\beta \in \mathrm{B}}$, where the $\rho_{\beta}$ are now real valued functions. Thus, locally $\mathrm{P}(r)=\sum_{\beta \in \mathrm{B}} \rho_{\beta}(r) \zeta_{\beta}$. But, the Unfold mapping is clearly $\mathbf{R}$-linear, thus denoting $\xi_{\beta}=\operatorname{Unfold}\left(\zeta_{\beta}\right)$ we get locally $\operatorname{Unfold}(\mathrm{P}(r))=$ $\sum_{\beta \in \mathrm{B}} \rho_{\beta}(r) \xi_{\beta}$, where the $\rho_{\beta}$ are real smooth functions and the $\xi_{\beta}$ are elements of $\mathcal{H}_{\mathbf{R}}$. Therefore Unfold $\circ \mathrm{P}$ is a plot of $\mathcal{H}_{\mathbf{R}}$ and Unfold is differentiable. In conclusion $\mathcal{H}$ and $\mathcal{H}_{\mathbf{R}}$ are diffeomorphic.

STEP 2: The infinite sphere is diffeomorphic to the real infinite sphere. Let us consider $\mathrm{X} \in \mathcal{H}_{\mathbf{R}}$, and $\mathrm{Z}=\operatorname{Fold}(\mathrm{X})$. Now, $\mathrm{Z}_{k}=\mathrm{X}_{2 k-1}+i \mathrm{X}_{2 k}$, hence $\left|Z_{k}\right|^{2}=\mathrm{X}_{2 k-1}^{2}+\mathrm{X}_{2 k}^{2}$. And, therefore $\|\mathrm{Z}\|^{2}=\sum_{k=1}^{\infty}\left\|\mathrm{Z}_{k}\right\|^{2}=\left(\mathrm{X}_{1}^{2}+\mathrm{X}_{2}^{2}\right)+\left(\mathrm{X}_{3}^{2}+\mathrm{X}_{4}^{2}\right)+\cdots=\sum_{k=1}^{\infty} \mathrm{X}_{k}^{2}=\|\mathrm{X}\|^{2}$. In conclusion, Fold sends the real infinite sphere

$$
\mathcal{S}_{\mathbf{R}}=\left\{\mathrm{X} \in \mathcal{H}_{\mathbf{R}} \mid \mathrm{X} \cdot \mathrm{X}=\sum_{k=1}^{\infty} \mathrm{X}_{k}^{2}=1\right\}
$$

onto $\mathcal{S}$. Now, since Fold is a diffeomorphism from $\mathcal{H}_{\mathbf{R}}$ to $\mathcal{H}$ it follows that its restriction to $\mathcal{S}_{\mathbf{R}}$ is a diffeomorphism onto $\mathcal{S}$, where $\mathcal{S}_{\mathbf{R}}$ and $\mathcal{S}$ are equipped with the subset diffeology.

STEP 3: The real infinite sphere as a diffeological manifold. Let us consider the following stereographic maps:

$$
\mathrm{F}_{+}: \mathcal{H}_{\mathbf{R}} \rightarrow \mathcal{S}_{\mathbf{R}} \quad \text { with } \quad \mathrm{F}_{+}: \xi \mapsto \mathrm{X}=\frac{1}{\|\xi\|^{2}+1}\left(\begin{array}{c}
\|\xi\|^{2}-1 \\
2 \xi
\end{array}\right)
$$

and

$$
\mathrm{F}_{-}: \mathcal{H}_{\mathbf{R}} \rightarrow \mathcal{S}_{\mathbf{R}} \quad \text { with } \quad \mathrm{F}_{-}: \xi \mapsto \mathrm{X}=\frac{1}{1+\|\xi\|^{2}}\left(\begin{array}{c}
1-\|\xi\|^{2} \\
2 \xi
\end{array}\right)
$$

where the matrix notation denotes the corresponding sequence in $\mathcal{H}_{\mathbf{R}}$

$$
\left(\begin{array}{l}
a \\
\xi
\end{array}\right) \sim\left(a, \xi_{1}, \xi_{2}, \ldots\right) \text { where } a \in \mathbf{R} \quad \xi=\left(\xi_{1}, \xi_{2}, \ldots\right) \in \mathcal{H}_{\mathbf{R}} .
$$

The maps $\mathrm{F}_{+}$and $\mathrm{F}_{-}$are stereographic maps of $\mathcal{S}_{\mathbf{R}}$, the image of $\mathrm{F}_{+}$is $\mathcal{S}_{\mathbf{R}}-\left\{\mathbf{e}_{1}\right\}$, and the image of $F_{-}$is $\mathcal{S}_{\mathbf{R}}-\left\{-\mathbf{e}_{1}\right\}$, where $\mathbf{e}_{1}$ is the vector whose first coordinate is 1 and 
the others are zero. More generally we shall denote by $\mathbf{e}_{k}$ the vector defined by:

$$
\operatorname{pr}_{k}\left(\mathbf{e}_{k}\right)=1 \text { and } \operatorname{pr}_{j}\left(\mathbf{e}_{k}\right)=0 \text { if } j \neq k .
$$

Let us denote, now, for any $\mathrm{X} \in \mathcal{H}_{\mathbf{R}}$ :

$$
\mathrm{X}=\left(\mathrm{X}_{1}, \mathrm{X}_{+}\right) \quad \text { with } \quad \mathrm{X}_{1}=\operatorname{pr}_{1}(\mathrm{X}) \quad \text { and } \quad \mathrm{X}_{+}=\left(\mathrm{X}_{2}, \mathrm{X}_{3}, \ldots\right) \in \mathcal{H}_{\mathbf{R}} \text {. }
$$

The inverse maps are given by:

$$
\mathrm{F}_{+}^{-1}: \mathcal{S}_{\mathbf{R}}-\left\{\mathbf{e}_{1}\right\} \rightarrow \mathcal{H}_{\mathbf{R}} \quad \text { with } \quad \mathrm{F}_{+}^{-1}: \mathrm{X} \mapsto \xi=\frac{\mathrm{X}_{+}}{1-\mathrm{X}_{1}}
$$

and

$$
\mathrm{F}_{-}^{-1}: \mathcal{S}_{\mathbf{R}}-\left\{-\mathbf{e}_{1}\right\} \rightarrow \mathcal{H}_{\mathbf{R}} \quad \text { with } \quad \mathrm{F}_{-}^{-1}: \mathrm{X} \mapsto \xi=\frac{\mathrm{X}_{+}}{1+\mathrm{X}_{1}}
$$

Let us show now that the stereographic maps are local diffeomorphisms from $\mathcal{H}_{\mathbf{R}}$ to $\mathcal{S}_{\mathbf{R}}$. We consider only $\mathrm{F}_{+}$since the case $\mathrm{F}_{-}$is completely analogous. We already checked that $\mathrm{F}_{+}$is injective, and its domain is $\mathcal{H}$ which is obviously D-open in $\mathcal{H}$. We shall prove that $\mathcal{S}-\left\{\mathbf{e}_{1}\right\}$, the image of $\mathrm{F}_{+}$, is $\mathrm{D}$-open in $\mathcal{S}$. Then, we shall prove that $\mathrm{F}_{+}$is differentiable as well as $\mathrm{F}_{+}^{-1}$, defined on $\mathcal{S}-\left\{\mathbf{e}_{1}\right\}$ equipped with the subset diffeology. And finally, applying the criterion (art. 6.8), it will follow that $\mathrm{F}_{+}$is a local diffeomorphism.

a) The map $\mathrm{F}_{+}$is injective. We already exhibited $\mathrm{F}_{+}^{-1}$.

b) The map $\mathrm{F}_{+}$is differentiable. Let us consider a parametrization $\mathrm{P}: \mathrm{U} \longrightarrow \mathcal{H}_{\mathbf{R}}$. For any $r_{0} \in \mathrm{U}$ there exists an open superset $\mathrm{V}$ of $r_{0}$ in $\mathrm{U}$, a finite set of indices $\mathrm{A}$, and a local family $\left(\lambda_{\alpha}, \mathrm{X}_{\alpha}\right)_{\alpha \in \mathrm{A}}$ such that:

$$
\mathrm{P} \uparrow \mathrm{V}: r \mapsto \sum_{\alpha \in \mathrm{A}} \lambda_{\alpha}(r) \mathrm{X}_{\alpha}
$$

So,

$$
\mathrm{F}_{+} \circ(\mathrm{P} \uparrow \mathrm{V}): r \mapsto\left(\begin{array}{c}
\epsilon(r) \\
\sum_{\alpha \in \mathrm{A}} \mu_{\alpha}(r) \mathrm{X}_{\alpha}
\end{array}\right)
$$

with

$$
\epsilon(r)=\frac{\left\|\sum_{\alpha \in \mathrm{A}} \lambda_{\alpha}(r) \mathrm{X}_{\alpha}\right\|^{2}-1}{\left\|\sum_{\alpha \in \mathrm{A}} \lambda_{\alpha}(r) \mathrm{X}_{\alpha}\right\|^{2}+1} \quad \text { and } \quad \mu_{\alpha}(r)=\frac{2 \lambda_{\alpha}(r)}{\left\|\sum_{\beta \in \mathrm{A}} \lambda_{\beta}(r) \mathrm{X}_{\beta}\right\|^{2}+1}
$$

The denominator of $\epsilon$ and $\mu_{\alpha}$ never vanishes, hence the functions $\epsilon$ and $\mu_{\alpha}$ belong to $\mathrm{e}^{\infty}(\mathrm{V}, \mathbf{R})$. Now $\mathrm{F}_{+} \circ(\mathrm{P} \uparrow \mathrm{V})$ rewrites:

$$
\mathrm{F}_{+} \circ(\mathrm{P} \uparrow \mathrm{V})(r)=\epsilon(r)\left(\begin{array}{l}
1 \\
0
\end{array}\right)+\sum_{\alpha \in \mathrm{A}} \mu_{\alpha}(r)\left(\begin{array}{c}
0 \\
\mathrm{X}_{\alpha}
\end{array}\right)
$$

This exhibits the map $\mathrm{F}_{+} \circ(\mathrm{P} \uparrow \mathrm{V})$ as a finite linear combination of vectors of $\mathcal{H}_{\mathbf{R}}$ with smooth parametrizations of $\mathbf{R}$ as coefficients. Therefore, $\mathrm{F}_{+} \circ \mathrm{P}$ is a plot of $\mathcal{H}_{\mathbf{R}}$. Since $\mathrm{F}_{+} \circ \mathrm{P}$ takes its values in $\mathcal{S}_{\mathbf{R}}$, it is a plot of the subset diffeology of $\mathcal{S}_{\mathbf{R}} \subset \mathcal{H}_{\mathbf{R}}$, where $\mathcal{H}_{\mathbf{R}}$ is equipped with the fine diffeology. Hence, $\mathrm{F}_{+}$is differentiable.

c) The map $\mathrm{F}_{+}^{-1}$ is differentiable. Let $\mathrm{P}: \mathrm{U} \longrightarrow \mathcal{S}_{\mathbf{R}}-\left\{\mathbf{e}_{1}\right\}$ be a plot. For any $r_{0} \in \mathrm{U}$ there exists an open superset $\mathrm{V}$ of $r_{0}$ in $\mathrm{U}$, a finite set of indices $\mathrm{A}$, a local family $\left(\lambda_{\alpha}, \mathrm{X}_{\alpha}\right)_{\alpha \in \mathrm{A}}$ such that:

$$
\mathrm{P} \uparrow \mathrm{V}: r \mapsto \sum_{\alpha \in \mathrm{A}} \lambda_{\alpha}(r) \mathrm{X}_{\alpha}
$$


SO

$$
\mathrm{F}_{+}^{-1} \circ(\mathrm{P} \uparrow \mathrm{V}): r \mapsto \sum_{\alpha \in \mathrm{A}} \mu_{\alpha}(r) \mathrm{X}_{\alpha,+} \quad \text { with } \quad \mu_{\alpha}(r)=\frac{\lambda_{\alpha}(r)}{1-\sum_{\beta \in \mathrm{A}} \lambda_{\beta}(r) \mathrm{X}_{\beta, 1}} .
$$

Since the $\mathrm{X}_{\beta, 1}$ form a finite set of constant numbers, the parametrization $\sum_{\beta \in \mathrm{A}} \lambda_{\beta}(r) \mathrm{X}_{\beta, 1}$ is smooth, and never equal to 1 since $\mathrm{P}$ takes its values in $\mathcal{S}_{\mathbf{R}}-\left\{\mathbf{e}_{1}\right\}$. Thus, for each $\alpha \in \mathrm{A}$, $\mu_{\alpha}(r)$ is a smooth parametrization of $\mathbf{R}$. The parametrization $\mathrm{F}_{+}^{-1} \circ(\mathrm{P} \uparrow \mathrm{V})$ is clearly a finite linear combination of vectors of $\mathcal{H}_{\mathbf{R}}$ with coefficients smooth parametrizations of R. Hence, $\mathrm{F}_{+}^{-1} \circ(\mathrm{P} \uparrow \mathrm{V})$ is a plot of the fine diffeology of $\mathcal{H}_{\mathbf{R}}$. Now, $\mathrm{F}_{+}^{-1} \circ \mathrm{P}$ is locally, at each point of $\mathrm{U}$, a plot of $\mathcal{H}_{\mathbf{R}}$, so it is a plot of $\mathcal{H}_{\mathbf{R}}$. Therefore, $\mathrm{F}_{+}^{-1}$ is a differentiable map from $\mathcal{S}_{\mathbf{R}}-\left\{\mathbf{e}_{1}\right\}$ to $\mathcal{H}_{\mathbf{R}}$.

d) The subset $\mathcal{S}_{\mathbf{R}}-\left\{\mathbf{e}_{1}\right\}$ is open for the D-topology. Let us recall that a set is D-open if and only if its preimage by any plot is open. Let $\mathrm{P}: \mathrm{U} \rightarrow \mathcal{S}_{\mathbf{R}}$ be a plot, for any $r_{0} \in \mathrm{U}$ there exists an open superset $\mathrm{V}$ of $r_{0}$ in $\mathrm{U}$, a finite set of indices A, a local family $\left(\lambda_{\alpha}, \mathrm{X}_{\alpha}\right)_{\alpha \in \mathrm{A}}$ such that:

$$
\mathrm{P} \uparrow \mathrm{V}: r \mapsto \sum_{\alpha \in \mathrm{A}} \lambda_{\alpha}(r) \mathrm{X}_{\alpha}
$$

Hence,

$$
(\mathrm{P} \uparrow \mathrm{V})^{-1}\left(\mathcal{S}_{\mathbf{R}}-\left\{\mathbf{e}_{1}\right\}\right)=\left\{r \in \mathrm{V} \mid \sum_{\alpha \in \mathrm{A}} \lambda_{\alpha}(r) \mathrm{X}_{\alpha, 1} \neq 1\right\} .
$$

But the parametrization $\mathrm{P}_{\mathrm{V}}: r \mapsto \sum_{\alpha \in \mathrm{A}} \lambda_{\alpha}(r) \mathrm{X}_{\alpha, 1}$ is smooth, a fortiori continuous. So, the preimage of $\mathbf{R}-\{1\}$ is open. Thus, $\mathrm{P}^{-1}\left(\mathcal{S}_{\mathbf{R}}-\left\{\mathbf{e}_{1}\right\}\right)$ is an union of open sets of $\mathrm{U}$, and therefore open. Hence, $\mathrm{P}^{-1}\left(\mathcal{S}_{\mathbf{R}}-\left\{\mathbf{e}_{1}\right\}\right)$ is open for any plot $\mathrm{P}$, that is $\mathcal{S}_{\mathbf{R}}-\left\{\mathbf{e}_{1}\right\}$ is D-open.

In conclusion, the diffeology of the real infinite sphere $\mathcal{S}_{\mathbf{R}}$ is generated by $\mathrm{F}_{+}$and $\mathrm{F}_{-}$, therefore $\mathcal{S}_{\mathbf{R}}$ is, by definition (art. 15.1), a diffeological manifold, modeled on $\mathcal{H}_{\mathbf{R}}$. Now, since $\mathcal{H}_{\mathbf{R}}$ and $\mathcal{H}$ are diffeomorphic, $\mathcal{S}$ is a diffeological manifold modeled on $\mathcal{H}$. The first sentence of the proposition is proved.

STEP 4: The infinite sphere is contractible. Let us prove now that the infinite sphere $\mathcal{S}_{\mathbf{R}}$, equipped with the subset fine diffeology of $\mathcal{H}_{\mathbf{R}}$, is contractible. That is, there exists a differentiable path $\gamma$ of $\mathcal{C}^{\infty}\left(\mathcal{S}_{\mathbf{R}}\right)$ connecting the identity $\mathbf{1}_{\mathcal{S}}: \mathrm{X} \mapsto \mathrm{X}$ with the constant $\operatorname{map} \hat{\mathbf{e}}_{1}: \mathrm{X} \mapsto \mathbf{e}_{1}$.

The proof of this proposition, uses the following linear map, called the shift operator:

$$
\text { Shift }: \mathcal{H}_{\mathbf{R}} \rightarrow \mathcal{H}_{\mathbf{R}} \text {, with } \operatorname{Shift}(\mathrm{X})_{1}=0 \text { and } \operatorname{Shift}(\mathrm{X})_{k}=\mathrm{X}_{k-1}, k>1 \text {. }
$$

In other words $\operatorname{Shift}(\mathrm{X})=(0, \mathrm{X})=\left(0, \mathrm{X}_{1}, \mathrm{X}_{2}, \ldots\right)$. The shift operator is clearly a linear operator, hence is differentiable for the fine diffeology. It is injective and preserves the scalar product. It injects strictly the infinite sphere into an equator.

We prove the contractibility of the infinite sphere in two steps, first we shall show that the constant map $\hat{\mathbf{e}}_{1}$ is homotopic to the shift operator, and then, that the shift operator is homotopic to the identity $\mathbf{1}_{\mathcal{S}}$. Then, applying the smashing function (art. 8.3) to the pair of homotopies we get a path connecting the constant map to the identity. 
a) Homotopy between $\hat{\mathbf{e}}_{1}$ and Shift. Let us consider the following 1-parameter family of deformations:

$$
\text { for all } t \in \mathbf{R} \text {, for all } \mathrm{X} \in \mathcal{S}_{\mathbf{R}}, \quad \rho_{t}(\mathrm{X})=\cos \left(\frac{\pi t}{2}\right) \mathbf{e}_{1}+\sin \left(\frac{\pi t}{2}\right) \operatorname{Shift}(\mathrm{X}) \text {. }
$$

For any $t \in \mathbf{R}, \rho_{t}(\mathrm{X}) \in \mathcal{S}_{\mathbf{R}}$. Since addition and multiplication by a smooth function are differentiable, the map $(t, \mathrm{X}) \mapsto \rho_{t}(\mathrm{X})$ is differentiable. Thus, the map $t \mapsto \rho_{t}$ is a path of $\mathcal{C}^{\infty}\left(\mathcal{S}_{\mathbf{R}}\right)$ connecting $\hat{\mathbf{e}}_{1}$ and Shift, precisely:

$$
\rho_{0}=\hat{\mathbf{e}}_{1} \text { and } \rho_{1}=\text { Shift. }
$$

b) Homotopy between Shift and $\mathbf{1}_{\mathcal{S}}$. Let us consider the following 1-parameter family of deformations:

$$
\text { for all } t \in \mathbf{R} \text {, for all } \mathrm{X} \in \mathcal{H}_{\mathbf{R}}, \quad \sigma_{t}(\mathrm{X})=t \mathrm{X}+(1-t) \operatorname{Shift}(\mathrm{X}) \text {. }
$$

Note that $\operatorname{ker}\left(\sigma_{t}\right)=0$, this is clear for $t=0$, and for nonzero $t$ it follows inductively by observing that the condition $\sigma_{t}(\mathrm{X})=0$ writes $\left(\mathrm{X}_{1}, \mathrm{X}_{2}, \mathrm{X}_{3}, \ldots\right)=\frac{t-1}{t}\left(0, \mathrm{X}_{1}, \mathrm{X}_{2}, \ldots\right)$. In particular $\sigma_{t}$ is nowhere zero on the sphere, so we can define $\rho_{t}: \mathcal{S}_{\mathbf{R}} \longrightarrow \mathcal{S}_{\mathbf{R}}$ by:

$$
\rho_{t}(\mathrm{X})=\frac{1}{\left\|\sigma_{t}(\mathrm{X})\right\|} \sigma_{t}(\mathrm{X}) .
$$

Let us check that $(t, \mathrm{X}) \mapsto \rho_{t}(\mathrm{X})$ is differentiable. First of all, $(t, \mathrm{X}) \mapsto \sigma_{t}(\mathrm{X})$ is clearly differentiable. Since the scalar product is differentiable, it follows that $(t, \mathrm{X}) \mapsto\left\|\sigma_{t}(X)\right\|^{2}$ is differentiable; and because this map takes its values in $] 0, \infty[$, its square root is differentiable. In conclusion, $t \mapsto \rho_{t}$ is a path in $\mathcal{C}^{\infty}\left(\mathcal{S}_{\mathbf{R}}\right)$, and

$$
\rho_{0}=\text { Shift and } \rho_{1}=\mathbf{1}_{\mathcal{S}} \text {. }
$$

We proved, with a) and b) that $\mathcal{S}_{\mathbf{R}}$ is contractible. The whole proposition is thus proved.

17.3. Vanishing homotopy groups. Note that since $\mathcal{S}_{\mathbf{R}}$ is contractible, all its homotopy groups vanish:

$$
\text { for all } k \in \mathbf{N}^{\star}, \quad \pi_{k}\left(\mathcal{S}_{\mathbf{R}}\right)=\{0\} .
$$

This fact is known in the topological framework, we proved that it is also true in diffeology.

18. The infinite projective space. Let us recall some set theoretic constructions, today classic. Let us introduce:

$$
\mathbf{C}^{\star}=\mathbf{C}-\{0\} \quad \text { and } \quad \mathcal{H}^{\star}=\mathcal{H}-\{0\} .
$$

Now, let us consider the group $\mathbf{C}^{\star}$ acting on $\mathcal{H}^{\star}$ by multiplication

$$
\text { For all }(z, \mathrm{Z}) \in \mathbf{C}^{\star} \times \mathcal{H}^{\star}, \quad(z, \mathrm{Z}) \mapsto z \mathrm{Z} \in \mathcal{H}^{\star} .
$$

The quotient of $\mathcal{H}^{\star}$ by this action of $\mathbf{C}^{\star}$ is called the infinite complex projective space, or simply the infinite projective space, and it is denoted $\mathcal{P}$. The restriction of the action of $\mathrm{S}^{1}$ to $\mathcal{S} \subset \mathcal{H}^{\star}$ gives the following set theoretic equivalences

$$
\mathcal{P}=\mathcal{H}^{\star} / \mathbf{C}^{\star} \simeq \mathcal{S} / \mathrm{S}^{1} \simeq \mathcal{S}_{\mathbf{R}} / \mathrm{SO}(2, \mathbf{R}) .
$$

Indeed, the sphere $\mathcal{S}$ intersects every $\mathbf{C}^{\star}$-orbit in $\mathcal{H}^{\star}$. The trace on $\mathcal{S}$ of the action of $\mathbf{C}^{\star}$ is the action of $\mathrm{S}^{1}$ identified to the set of complex numbers of modulus 1 . Hence, 
$\mathcal{H}^{\star} / \mathbf{C}^{\star} \simeq \mathcal{S} / \mathrm{S}^{1}$. The last equivalence is obtained by identifying $\mathcal{H}$ to $\mathcal{H}_{\mathbf{R}}+i \mathcal{H}_{\mathbf{R}}$ or $\mathcal{H}_{\mathbf{R}} \times \mathcal{H}_{\mathbf{R}}$, thanks to the unique decomposition $\mathrm{Z}=\mathrm{X}+i \mathrm{Y}$, where $\mathrm{Z}$ belongs to $\mathcal{H}$ and $\mathrm{X}$ and $\mathrm{Y}$ belong to $\mathcal{H}_{\mathbf{R}}$. The action of $\mathrm{S}^{1} \sim \mathrm{SO}(2, \mathbf{R})$ on the pair $(\mathrm{X}, \mathrm{Y})$ is given by:

$$
\left(\begin{array}{cc}
\cos (t) & -\sin (t) \\
\sin (t) & \cos (t)
\end{array}\right)\left(\begin{array}{l}
\mathrm{X} \\
\mathrm{Y}
\end{array}\right)=\left(\begin{array}{c}
\cos (t) \mathrm{X}-\sin (t) \mathrm{Y} \\
\sin (t) \mathrm{X}+\cos (t) \mathrm{Y}
\end{array}\right)
$$

Now, let us equip the infinite projective space $\mathcal{P}$ with the quotient diffeology of the subset diffeology of $\mathcal{H}^{\star} \subset \mathcal{H}$. We shall see that these identifications are also valid from the diffeological point of view. That is, $\mathcal{H}^{\star} / \mathbf{C}^{\star}, \mathcal{S} / \mathrm{S}^{1}$ and $\mathcal{S}_{\mathbf{R}} / \mathrm{SO}(2, \mathbf{R})$ are diffeomorphic.

18.1. The infinite projective space as a diffeological space. The infinite projective space $\mathcal{P}$ is a diffeological manifold modeled on $\mathcal{H}$. The projection $\pi: \mathcal{H}^{\star} \rightarrow \mathcal{P}$ is a $\mathbf{C}^{\star}$ principal diffeological fibration, locally trivial. The projective space $\mathcal{P}$ is naturally diffeomorphic to the quotient $\mathcal{S} / \mathcal{S}^{1}$, moreover the restriction $\pi_{\mathcal{S}}$ of $\pi: \mathcal{H}^{\star} \rightarrow \mathcal{P}$ to the sphere $\mathcal{S}$ is a principal $\mathrm{S}^{1}$ diffeological fibration, locally trivial. The homotopy of $\mathcal{P}$ is given by:

$$
\pi_{2}(\mathcal{P})=\mathbf{Z} \text { and } \pi_{k}(\mathcal{P})=\{0\}, \text { if } k \neq 2 .
$$

The principal fibrations $\pi$ or $\pi_{\mathcal{S}}$ are not trivial.

Proof. $\mathcal{P}$ is a diffeological manifold, modeled on $\mathcal{H}$. Let us denote by $[\mathrm{Z}]=\left[\mathrm{Z}_{1}, \mathrm{Z}_{2}, \ldots\right]$ the class in $\mathcal{P}$ of an element $\mathrm{Z}=\left(\mathrm{Z}_{1}, \mathrm{Z}_{2}, \ldots\right)$ of $\mathcal{H}$. Let us consider the various affine subspaces $\mathcal{H}_{k} \subset \mathcal{H}$ defined by:

$$
\mathcal{H}_{k}=\left\{\mathrm{Z} \in \mathcal{H} \mid \mathrm{Z}_{k}=1\right\}, k \in \mathbf{N}^{\star} .
$$

These spaces intersect the orbits of the group $\mathbf{C}^{\star}$ in one and only one point. The orbits which do not meet $\mathcal{H}_{k}$ are those such that $\mathrm{Z}_{k}=0$. However, the orbit of any point $\mathrm{Z} \in \mathcal{H}^{\star}$ intersects some $\mathcal{H}_{k}$, in other words $\cup_{k \in \mathbf{N}^{\star}} \mathbf{C}^{\star} \mathcal{H}_{k}=\mathcal{H}^{\star}$, or $\pi\left(\cup_{k \in \mathbf{N}^{\star}} \mathcal{H}_{k}\right)=\mathcal{P}$. For each $k \in \mathbf{N}^{\star}$ let us define the injection $j_{k}: \mathcal{H} \longrightarrow \mathcal{H}^{\star}$ by

$$
j_{1}(\mathrm{Z})=(1, \mathrm{Z}) \quad \text { and } \quad j_{k}(\mathrm{Z})=\left(\mathrm{Z}_{1}, \ldots, \mathrm{Z}_{k-1}, 1, \mathrm{Z}_{k}, \ldots\right), \text { if } k>1 .
$$

And let us define also the map

$$
\mathrm{F}_{k}=\pi \circ j_{k}, \mathrm{~F}_{k}: \mathcal{H} \longrightarrow \mathcal{P} .
$$

We shall prove now that the $\mathrm{F}_{k}$ are inductions onto D-open sets of $\mathcal{P}$, and therefore are local diffeomorphisms (art. 6.8):

a) The map $j_{k}$ is an induction. Let us consider a plot $\mathrm{P}$ of $\mathcal{H}$ with values in $\mathcal{H}_{k}$, locally:

$$
\mathrm{P}(r)=\sum_{\alpha \in \mathrm{A}} \lambda_{\alpha}(r) \mathrm{Z}_{\alpha} \text { and } \mathrm{P}_{k}(r)=\sum_{\alpha \in \mathrm{A}} \lambda_{\alpha}(r) \mathrm{Z}_{\alpha, k}=1, \quad \mathrm{P}_{k}=\operatorname{pr}_{k} \circ \mathrm{P} .
$$

Let us define $\zeta_{\alpha}$ by:

$$
\zeta_{\alpha}=\left(\mathrm{Z}_{\alpha, 1}, \ldots, \mathrm{Z}_{\alpha, k-1}, 1, \mathrm{Z}_{\alpha, k+1}, \ldots\right)
$$

For each $\alpha$ in $\mathrm{A}, \zeta_{\alpha}$ belongs to $\mathcal{H}_{k}$. Let $\mathbf{e}_{k}$ be the sequence with only one nonzero term, equal to 1 , at the place $k$. From the condition above, we have locally: 


$$
\begin{aligned}
\mathrm{P}(r) & =\sum_{\alpha \in \mathrm{A}} \lambda_{\alpha}(r) \zeta_{\alpha}-\sum_{\alpha \in \mathrm{A}} \lambda_{\alpha}(r) \mathbf{e}_{k}+\sum_{\alpha \in \mathrm{A}} \lambda_{\alpha}(r) \mathrm{Z}_{\alpha, k} \mathbf{e}_{k} \\
& =\sum_{\alpha \in \mathrm{A}} \lambda_{\alpha}(r) \zeta_{\alpha}+\left(1-\sum_{\alpha \in \mathrm{A}} \lambda_{\alpha}(r)\right) \mathbf{e}_{k} .
\end{aligned}
$$

Now, since the vectors $\zeta_{\alpha}$ and $\mathbf{e}_{k}$ belong to $\mathcal{H}_{k}$, the plot $j_{k}^{-1} \circ \mathrm{P}$ writes locally

$$
j_{k}^{-1} \circ \mathrm{P}(r)=\sum_{\alpha \in \mathrm{A}} \lambda_{\alpha}(r) j^{-1}\left(\zeta_{\alpha}\right)+\left(1-\sum_{\alpha \in \mathrm{A}} \lambda_{\alpha}(r)\right) j^{-1}\left(\mathbf{e}_{k}\right)
$$

but $j_{k}(0)=\mathbf{e}_{k}$ implies $j_{k}^{-1}\left(\mathbf{e}_{k}\right)=0$, hence:

$$
j_{k}^{-1} \circ \mathrm{P}(r)=\sum_{\alpha \in \mathrm{A}} \lambda_{\alpha}(r) j^{-1}\left(\zeta_{\alpha}\right) .
$$

This exhibits the parametrization $j_{k}^{-1} \circ \mathrm{P}$ as a plot of $\mathcal{H}$, hence $j_{k}$ is an induction.

b) The map $F_{k}$ is clearly differentiable and injective.

c) Let $\mathrm{Q}: \mathrm{U} \rightarrow \mathcal{P}$ be a plot with values in $\mathrm{F}_{k}(\mathcal{H})$, and let $r_{0} \in \mathrm{U}$. By definition of the quotient diffeology, there exists a superset $\mathrm{V}$ of $r_{0}$ and a plot $\mathrm{P}: \mathrm{V} \rightarrow \mathcal{H}^{\star}$ such that $\mathrm{Q} \uparrow$ $\mathrm{V}=\pi \circ \mathrm{P}$. By hypothesis, for each $r \in \mathrm{V}, \mathrm{P}_{k}(r) \neq 0$, where $\mathrm{P}_{k}=\mathrm{pr}_{k} \circ \mathrm{P}$, therefore $\mathrm{P}^{\prime}: \mathrm{V}$ $\longrightarrow \mathcal{H}^{\star}$ defined by $\mathrm{P}^{\prime}(r)=\mathrm{P}(r) / \mathrm{P}_{k}(r)$ takes its values in $\mathcal{H}_{k}$. Since $\mathrm{P}_{k}$ is differentiable, $\mathrm{P}^{\prime}$ is differentiable and $\mathrm{Q} \uparrow \mathrm{V}=\pi \circ \mathrm{P}^{\prime}$. The plot $\mathrm{P}^{\prime}$ takes its values in $\mathcal{H}_{k}$, and $j_{k}$ is an induction, so the composition $j_{k}^{-1} \circ \mathrm{P}^{\prime}$ is a plot of $\mathcal{H}$. But, by construction, $j_{k}^{-1} \circ \mathrm{P}^{\prime}=\mathrm{F}_{k}^{-1} \circ \mathrm{Q}$, thus $\mathrm{F}_{k}^{-1} \circ \mathrm{Q}$ is a plot of $\mathcal{H}$ and $\mathrm{F}_{k}^{-1}$ is differentiable. Therefore, $\mathrm{F}_{k}$ is an induction.

d) Now let us prove that the image of $\mathrm{F}_{k}$ is D-open. Since the D-topology of the quotient diffeology is the quotient topology of the D-topology (art. 6.5), it is enough to prove that the preimage by $\pi$ of the $\mathrm{F}_{k}(\mathcal{H})$ is D-open in $\mathcal{H}^{\star}$. We saw that $\pi^{-1}\left(\mathrm{~F}_{k}(\mathcal{H})\right)$ is the set of all $\mathrm{Z} \in \mathcal{H}$ such that $\mathrm{Z}_{k} \neq 0$, i.e. $\operatorname{pr}_{k}^{-1}\left(\mathbf{C}^{\star}\right)$. But $\operatorname{pr}_{k}$ is linear, hence smooth, hence continuous. Since $\mathbf{C}^{\star}$ is open it follows that $\pi^{-1}\left(\mathrm{~F}_{k}(\mathcal{H})\right)$ is open.

So, we just proved that the $\mathrm{F}_{k}$ are local diffeomorphisms. Since their images cover $\mathcal{P}$, the space $\mathcal{P}$ is a diffeological manifold modeled on $\mathcal{H}$.

Let us prove now, that $\pi$ is a $\mathbf{C}^{\star}$ principal fibration. Let us consider the map

$$
\Phi_{k}: \mathcal{H} \times \mathbf{C}^{\star} \longrightarrow \mathcal{H}^{\star} \text { such that } \Phi_{k}(\mathrm{Z}, z)=z j_{k}(\mathrm{Z}) .
$$

The previous part of the proof proved that $\Phi_{k}$ is a diffeomorphism covering $\mathrm{F}_{k}$. It commutes with the action of $\mathbf{C}^{\star}$. Thus, the projection $\pi$ is a diffeological $\mathbf{C}^{\star}$ principal bundles, locally trivial.

The restriction $\pi_{\mathcal{S}}: \mathcal{S} \longrightarrow \mathcal{P}$ is a diffeological fibration. The restriction to the infinite sphere has been described above. Just the action of $S^{1}$ remains from the action of $\mathbf{C}^{\star}$. Now, thanks to the projection $\mathrm{Z} \mapsto \mathrm{Z} /\|\mathrm{Z}\|$, the natural bijection $\mathcal{S} / \mathrm{S}^{1} \rightarrow \mathcal{P}=\mathcal{H}^{\star} / \mathbf{C}^{\star}$ is a diffeomorphism (art. 5.6). The projection $\pi$ to $\mathcal{S}$ is a reduction of the fibration $\mathcal{H}^{\star}$ $\rightarrow \mathcal{P}$ to the subgroup $\mathrm{S}^{1} \subset \mathbf{C}^{\star}$, therefore it is a fibration [Ig185].

The homotopy of $\mathcal{P}$. We apply the exact sequence of homotopy for diffeological bundle (art. 9.2), since the total space $\mathcal{S}$ is contractible we get $\pi_{k}(\mathcal{P})=\pi_{k-1}\left(\mathrm{~S}^{1}\right)$, which gives the homotopy groups of $\mathcal{P}$, in particular the only nontrivial one: $\pi_{2}(\mathcal{P})=\pi_{1}\left(\mathrm{~S}^{1}\right)=\mathbf{Z}$.

The fibration is not trivial. Indeed, otherwise the first homotopy group $\pi_{1}(\mathcal{S})$ would be equal to $\pi_{1}\left(\mathcal{P} \times \mathrm{S}^{1}\right)=\pi_{1}(\mathcal{P}) \times \pi_{1}\left(\mathrm{~S}^{1}\right)=\{0\} \times \mathbf{Z}=\mathbf{Z}$ which is not the case. 


\section{THE SYMPLECTIC PICTURE}

In this chapter we introduce a homogeneous symplectic form $\Omega$ on the square $\mathcal{H}_{\mathbf{R}}^{2}=$ $\mathcal{H}_{\mathbf{R}} \times \mathcal{H}_{\mathbf{R}}$, which we call the standard symplectic form. This differential form is invariant by translation. The form $\Omega$ is the exterior derivative of a 1-form $\Lambda$, called the Liouville form, because it is the restriction to $\mathcal{H}_{\mathbf{R}}^{2}$ of the general Liouville's form defined on the 1-form bundle of any diffeological space [PIZ05]. The restriction $\varpi$ of $\Lambda$ to the infinite sphere $\mathcal{S}_{\mathbf{R}}$ is a connection 1-form, for the natural action of the group $\operatorname{SO}(2, \mathbf{R})$ defining the Hopf fibration. The curvature of $\varpi$ is a closed 2 -form $\omega$ defined on $\mathcal{P} \sim \mathcal{S}_{\mathbf{R}} / \operatorname{SO}(2, \mathbf{R})$, which generalizes the standard Fubini-Study form of the finite dimensional construction.

19. The Liouville form on the Hilbert square. The following proposition gives the definition of the Liouville form on the square $\mathcal{H}_{\mathbf{R}}^{2}$, a natural extension of the Liouville form of the finite dimensional case $\mathbf{R}^{2 n}$.

19.1. The Liouville form. Let $\mathrm{P}: \mathrm{U} \rightarrow \mathcal{H}_{\mathbf{R}} \times \mathcal{H}_{\mathbf{R}}$ be a plot, $\mathrm{V} \subset \mathrm{U}$ an open subset such that there exists a finite local family $\left(\lambda_{\alpha},\left(\mathrm{X}_{\alpha}, \mathrm{Y}_{\alpha}\right)\right)$, where the $\lambda_{\alpha}$ are smooth real functions defined on $\mathrm{V}$, and the $\left(\mathrm{X}_{\alpha}, \mathrm{Y}_{\alpha}\right)$ are vectors of $\mathcal{H}_{\mathbf{R}} \times \mathcal{H}_{\mathbf{R}}$, such that:

$$
\mathrm{P} \uparrow \mathrm{V}: r \mapsto \sum_{\alpha \in \mathrm{A}} \lambda_{\alpha}(r)\left(\mathrm{X}_{\alpha}, \mathrm{Y}_{\alpha}\right)
$$

Let $\Lambda(\mathrm{P} \uparrow \mathrm{V})$ be the following 1-form, defined on $\mathrm{V}$ :

$$
\Lambda(\mathrm{P} \uparrow \mathrm{V})=\frac{1}{2} \sum_{\alpha, \beta \in \mathrm{A}}\left(\mathrm{X}_{\alpha} \cdot \mathrm{Y}_{\beta}-\mathrm{Y}_{\alpha} \cdot \mathrm{X}_{\beta}\right)\left(\lambda_{\alpha} d \lambda_{\beta}-\lambda_{\beta} d \lambda_{\alpha}\right)
$$

1. If $\mathrm{P}^{\prime}$ is any plot of $\mathcal{H}_{\mathbf{R}} \times \mathcal{H}_{\mathbf{R}}$ such that $\mathrm{P} \uparrow \mathrm{V}=\mathrm{P}^{\prime} \uparrow \mathrm{V}$, then $\Lambda(\mathrm{P} \uparrow \mathrm{V})=\Lambda\left(\mathrm{P}^{\prime} \uparrow \mathrm{V}\right)$.

2. There exists a 1 -form $\Lambda(\mathrm{P})$ on $\mathrm{U}$ such that for any open subset $\mathrm{V} \subset \mathrm{U}, \Lambda(\mathrm{P} \uparrow \mathrm{V})=$ $\Lambda(\mathrm{P}) \uparrow \mathrm{V}$.

3. The map $\Lambda: \mathrm{P} \mapsto \Lambda(\mathrm{P})$ is a 1 -form of $\mathcal{H}_{\mathbf{R}} \times \mathcal{H}_{\mathbf{R}}$.

Proof. Let us prove successively:

1. Let us develop the restriction of $\mathrm{P}^{\prime}$ to $\mathrm{V}$,

$$
\mathrm{P}^{\prime} \uparrow \mathrm{V}: r \mapsto \sum_{\alpha^{\prime} \in A^{\prime}} \lambda_{\alpha^{\prime}}^{\prime}(r)\left(\mathrm{X}_{\alpha^{\prime}}^{\prime}, \mathrm{Y}_{\alpha^{\prime}}^{\prime}\right)
$$

Then, $\mathrm{P} \uparrow \mathrm{V}=\mathrm{P}^{\prime} \uparrow \mathrm{V}$ implies

$$
\sum_{\alpha \in \mathrm{A}} \lambda_{\alpha} \mathrm{X}_{\alpha}=\sum_{\alpha^{\prime} \in A^{\prime}} \lambda_{\alpha^{\prime}}^{\prime} \mathrm{X}_{\alpha^{\prime}}^{\prime} \quad \text { and } \quad \sum_{\alpha \in \mathrm{A}} \lambda_{\alpha} \mathrm{Y}_{\alpha}=\sum_{\alpha^{\prime} \in A^{\prime}} \lambda_{\alpha^{\prime}}^{\prime} \mathrm{Y}_{\alpha^{\prime}}^{\prime}
$$

Let us note that:

$$
\Lambda(\mathrm{P} \uparrow \mathrm{V})=\left(\sum_{\alpha \in \mathrm{A}} \lambda_{\alpha} \mathrm{X}_{\alpha}\right) \cdot\left(\sum_{\beta \in \mathrm{A}} d \lambda_{\beta} \mathrm{Y}_{\beta}\right)-\left(\sum_{\alpha \in \mathrm{A}} \lambda_{\alpha} \mathrm{Y}_{\alpha}\right) \cdot\left(\sum_{\beta \in \mathrm{A}} d \lambda_{\beta} \mathrm{X}_{\beta}\right)
$$

Thus, we have:

$$
\begin{aligned}
\Lambda(\mathrm{P} \uparrow \mathrm{V})-\Lambda\left(\mathrm{P}^{\prime} \uparrow \mathrm{V}\right) & =\left(\sum_{\alpha \in \mathrm{A}} \lambda_{\alpha} \mathrm{X}_{\alpha}\right) \cdot\left(\sum_{\alpha^{\prime \prime} \in \mathrm{A}^{\prime \prime}} d \lambda_{\alpha^{\prime \prime}}^{\prime \prime} \mathrm{Y}_{\alpha^{\prime \prime}}^{\prime \prime}\right) \\
& -\left(\sum_{\alpha \in \mathrm{A}} \lambda_{\alpha} \mathrm{Y}_{\alpha}\right) \cdot\left(\sum_{\alpha^{\prime \prime} \in \mathrm{A}^{\prime \prime}} d \lambda_{\alpha^{\prime \prime}}^{\prime \prime} \mathrm{X}_{\alpha^{\prime \prime}}^{\prime \prime}\right)
\end{aligned}
$$


where $\mathrm{A}^{\prime \prime}$ is the following reordering of the two sets of indices $\mathrm{A}$ and $\mathrm{A}^{\prime}, \lambda_{\alpha^{\prime \prime}}^{\prime \prime}, \mathrm{X}_{\alpha^{\prime \prime}}^{\prime \prime}$ and $\mathrm{Y}_{\alpha^{\prime \prime}}^{\prime \prime}$ following this reordering: let $\mathrm{A}=\{1, \ldots, a\}$ and $\mathrm{A}^{\prime}=\left\{1, \ldots, a^{\prime}\right\}$ we denote $\mathrm{A}^{\prime \prime}=\left\{1, \ldots, a^{\prime \prime}\right\}$ such that $a^{\prime \prime}=a+a^{\prime}$ and:

$$
\begin{array}{rll}
\lambda_{\alpha^{\prime \prime}}^{\prime \prime}=\lambda_{\alpha} \text { if } 1 \leq \alpha^{\prime \prime} \leq a & \text { and } & \lambda_{\alpha^{\prime \prime}}^{\prime \prime}=\lambda_{\alpha^{\prime}}^{\prime} \text { if } a+1 \leq \alpha^{\prime \prime} \leq a+a^{\prime} \\
\mathrm{Y}_{\alpha^{\prime \prime}}^{\prime \prime}=\mathrm{Y}_{\alpha} \text { if } 1 \leq \alpha^{\prime \prime} \leq a & \text { and } & \mathrm{Y}_{\alpha^{\prime \prime}}^{\prime \prime}=-\mathrm{Y}_{\alpha^{\prime}}^{\prime} \text { if } a+1 \leq \alpha^{\prime \prime} \leq a+a^{\prime} \\
\mathrm{X}_{\alpha^{\prime \prime}}^{\prime \prime}=\mathrm{X}_{\alpha} \text { if } 1 \leq \alpha^{\prime \prime} \leq a & \text { and } & \mathrm{X}_{\alpha^{\prime \prime}}^{\prime \prime}=-\mathrm{X}_{\alpha^{\prime}}^{\prime} \text { if } a+1 \leq \alpha^{\prime \prime} \leq a+a^{\prime}
\end{array}
$$

With this reordering we get:

$$
\begin{aligned}
& \sum_{\alpha \in \mathrm{A}} \lambda_{\alpha} \mathrm{Y}_{\alpha}=\sum_{\alpha^{\prime} \in \mathrm{A}^{\prime}} \lambda_{\alpha^{\prime}}^{\prime} \mathrm{Y}_{\alpha^{\prime}}^{\prime} \Rightarrow \sum_{\alpha^{\prime \prime} \in \mathrm{A}^{\prime \prime}} \lambda_{\alpha^{\prime \prime}}^{\prime \prime} \mathrm{Y}_{\alpha^{\prime \prime}}^{\prime \prime}=0, \\
& \sum_{\alpha \in \mathrm{A}} \lambda_{\alpha} \mathrm{X}_{\alpha}=\sum_{\alpha^{\prime} \in \mathrm{A}^{\prime}} \lambda_{\alpha^{\prime}}^{\prime} \mathrm{X}_{\alpha^{\prime}}^{\prime} \Rightarrow \sum_{\alpha^{\prime \prime} \in \mathrm{A}^{\prime \prime}} \lambda_{\alpha^{\prime \prime}}^{\prime \prime} \mathrm{X}_{\alpha^{\prime \prime}}^{\prime \prime}=0 .
\end{aligned}
$$

Let us project this vector on each factor $\mathbf{R}$ by the projection $\mathrm{pr}_{k}$ :

$$
\begin{gathered}
\sum_{\alpha^{\prime \prime} \in \mathrm{A}^{\prime \prime}} \lambda_{\alpha^{\prime \prime}}^{\prime \prime} \mathrm{Y}_{\alpha^{\prime \prime}}^{\prime \prime}=0 \quad \\
\sum_{\alpha^{\prime \prime} \in \mathrm{A}^{\prime \prime}} \lambda_{\alpha^{\prime \prime}}^{\prime \prime} \mathrm{X}_{\alpha^{\prime \prime}}^{\prime \prime}=0
\end{gathered}
$$

But $\mathrm{X}_{\alpha^{\prime \prime}, k}^{\prime \prime}$ and $\mathrm{Y}_{\alpha^{\prime \prime}, k}^{\prime \prime}$ are just numbers, $\sum_{\alpha^{\prime \prime} \in A^{\prime \prime}} \lambda_{\alpha^{\prime \prime}}^{\prime \prime} \mathrm{X}_{\alpha^{\prime \prime}, k}^{\prime \prime}$ and $\sum_{\alpha^{\prime \prime} \in A^{\prime \prime}} \lambda_{\alpha^{\prime \prime}}^{\prime \prime} \mathrm{Y}_{\alpha^{\prime \prime}, k}^{\prime \prime}$ are smooth functions of $r \in \mathrm{V}$. Since these functions vanish identically, so do their derivatives, with respect to $r$. And we get:

$$
\begin{aligned}
& \text { for all } k \in \mathbf{N}, \sum_{\alpha^{\prime \prime} \in \mathrm{A}^{\prime \prime}} d \lambda_{\alpha^{\prime \prime}}^{\prime \prime} \mathrm{Y}_{\alpha^{\prime \prime}, k}^{\prime \prime}=0 \quad \Rightarrow \quad \sum_{\alpha^{\prime \prime} \in \mathrm{A}^{\prime \prime}} d \lambda_{\alpha^{\prime \prime}}^{\prime \prime} \mathrm{Y}_{\alpha^{\prime \prime}}^{\prime \prime}=0, \\
& \text { for all } k \in \mathbf{N}, \sum_{\alpha^{\prime \prime} \in \mathrm{A}^{\prime \prime}} d \lambda_{\alpha^{\prime \prime}}^{\prime \prime} \mathrm{X}_{\alpha^{\prime \prime}, k}^{\prime \prime}=0 \Rightarrow \sum_{\alpha^{\prime \prime} \in \mathrm{A}^{\prime \prime}} d \lambda_{\alpha^{\prime \prime}}^{\prime \prime} \mathrm{X}_{\alpha^{\prime \prime}}^{\prime \prime}=0 .
\end{aligned}
$$

And finally $\Lambda(\mathrm{P} \uparrow \mathrm{V})=\Lambda\left(\mathrm{P}^{\prime} \uparrow \mathrm{V}\right)$.

2. Let us consider a covering $\mathrm{U}_{i}$ of $\mathrm{U}$ such that the plot $\mathrm{P}$, restricted to each $\mathrm{U}_{i}$, is the sum of a finite linear combination of vectors with smooth parametrizations as coefficients. Let $i$ and $j$ be two indices of the covering, let us denote $\mathrm{P}_{i}=\mathrm{P} \uparrow \mathrm{U}_{i}$. By the previous statement we have

$$
\Lambda\left(\mathrm{P}_{i}\right) \uparrow \mathrm{U}_{i} \cap \mathrm{U}_{j}=\Lambda\left(\mathrm{P}_{j}\right) \uparrow \mathrm{U}_{i} \cap \mathrm{U}_{j}
$$

Because a differential form is local (art. 10.4), there exists a 1-form $\Lambda(\mathrm{P})=\sup _{i} \Lambda\left(\mathrm{P}_{i}\right)$, defined on $\mathrm{U}$ such that $\Lambda(\mathrm{P}) \uparrow \mathrm{U}_{i}=\Lambda\left(\mathrm{P}_{i}\right)$

3. It remains to show that the map $\Lambda$ is a 1 -form on $\mathcal{H}_{\mathbf{R}} \times \mathcal{H}_{\mathbf{R}}$. That is, to check that for any plot $\mathrm{P}: \mathrm{U} \longrightarrow \mathcal{H}_{\mathbf{R}} \times \mathcal{H}_{\mathbf{R}}$, and for any smooth parametrization $\mathrm{F}: \mathrm{U}^{\prime} \longrightarrow \mathrm{U}$, $\Lambda(\mathrm{P} \circ \mathrm{F})=\mathrm{F}^{*}(\Lambda(\mathrm{P}))$. Let $r_{0}^{\prime} \in \mathrm{U}^{\prime}$ and $r_{0}=\mathrm{F}\left(r_{0}^{\prime}\right)$, let

$$
\mathrm{P} \uparrow \mathrm{V}: r \mapsto \sum_{\alpha \in \mathrm{A}} \lambda_{\alpha}(r)\left(\mathrm{X}_{\alpha}, \mathrm{Y}_{\alpha}\right)
$$


as usual. Let us define now $\mathrm{V}^{\prime}=\mathrm{F}^{-1}(\mathrm{~V})$ and $\lambda_{\alpha}^{\prime}=\lambda_{\alpha} \circ \mathrm{F}$, we have

$$
\begin{aligned}
\Lambda\left(\mathrm{P} \circ \mathrm{F} \uparrow \mathrm{V}^{\prime}\right)_{r^{\prime}}\left(\delta r^{\prime}\right) & =\sum_{\alpha, \beta \in \mathrm{A}} \mathrm{X}_{\alpha} \cdot \mathrm{Y}_{\beta} \lambda_{\alpha}^{\prime}\left(r^{\prime}\right) d \lambda_{\beta}^{\prime}\left(r^{\prime}\right)\left(\delta r^{\prime}\right) \\
& =\sum_{\alpha, \beta \in \mathrm{A}} \mathrm{X}_{\alpha} \cdot \mathrm{Y}_{\beta} \lambda_{\alpha}\left(\mathrm{F}\left(r^{\prime}\right)\right) d \lambda_{\beta}\left(\mathrm{F}\left(r^{\prime}\right)\right)\left(\mathrm{D}\left(\mathrm{F}_{r^{\prime}}\right)\left(\delta r^{\prime}\right)\right) \\
& =\sum_{\alpha, \beta \in \mathrm{A}} \mathrm{X}_{\alpha} \cdot \mathrm{Y}_{\beta} \lambda_{\alpha}(r) d \lambda_{\beta}(r)(\delta r),
\end{aligned}
$$

with $r=\mathrm{F}\left(r^{\prime}\right)$ and $\delta r=\mathrm{D}(\mathrm{F})_{r^{\prime}}\left(\delta r^{\prime}\right)$, this is the definition of the pull-back. Therefore, $\Lambda\left(\mathrm{P} \circ \mathrm{F} \uparrow \mathrm{V}^{\prime}\right)=\mathrm{F}^{*}(\Lambda(\mathrm{P} \uparrow \mathrm{V}))$. Since this is true locally, and since it's a local property, it's true globally and $\Lambda(\mathrm{P} \circ \mathrm{F})=\mathrm{F}^{*}(\Lambda(\mathrm{P}))$.

19.2. The complex picture of the Liouville form. Let us consider the identification of $\mathcal{H}$ with $\mathcal{H}_{\mathbf{R}} \times \mathcal{H}_{\mathbf{R}}$ defined by the unique decomposition $\mathrm{Z}=\mathrm{X}+i \mathrm{Y}$, with $(\mathrm{X}, \mathrm{Y}) \in \mathcal{H}_{\mathbf{R}} \times \mathcal{H}_{\mathbf{R}}$. Let $\mathrm{P}: r \mapsto \sum_{\alpha \in \mathrm{A}} \lambda_{\alpha}(r) \mathrm{Z}_{\alpha}$ be a plot of $\mathcal{H}$, where $\left(\lambda_{\alpha}, \mathrm{Z}_{\alpha}\right)_{\alpha \in \mathrm{A}}$ is a local family. The $\lambda_{\alpha}$ are complex valued functions and the $\mathrm{Z}_{\alpha}$ are vectors of $\mathcal{H}$. Let us define the symbol $d \mathrm{Z}$ by :

$$
d \mathrm{Z}(\mathrm{P}): r \mapsto \sum_{\alpha \in \mathrm{A}} d \lambda_{\alpha}(r) \mathrm{Z}_{\alpha} \quad \text { where } \quad \mathrm{P}: r \mapsto \sum_{\alpha \in \mathrm{A}} \lambda_{\alpha}(r) \mathrm{Z}_{\alpha} .
$$

Here $d \lambda_{\alpha}$ needs to be understood as

$$
d \lambda_{\alpha}=d a_{\alpha}+i d b_{\alpha}, \text { where } \lambda_{\alpha}=a_{\alpha}+i b_{\alpha} .
$$

Then, the Liouville form $\varpi$, pulled back on $\mathcal{H}$ by the isomorphism $\Phi: \mathrm{Z} \mapsto(\mathrm{X}, \mathrm{Y})$, writes:

$$
\Phi^{*}(\varpi)=\frac{1}{2 i}[\mathrm{Z} \cdot d \mathrm{Z}-d \mathrm{Z} \cdot \mathrm{Z}] .
$$

Proof. This identity is obtained just by developing the computation as follows:

$$
\begin{aligned}
(\mathrm{Z} \cdot d \mathrm{Z}-d \mathrm{Z} \cdot \mathrm{Z})(\mathrm{P}) & =\sum_{\alpha \in \mathrm{A}} \lambda_{\alpha}^{*}\left(\mathrm{X}_{\alpha}-i \mathrm{Y}_{\alpha}\right) \sum_{\beta \in \mathrm{A}} d \lambda_{\beta}\left(\mathrm{X}_{\beta}+i \mathrm{Y}_{\beta}\right) \\
& -\sum_{\alpha \in \mathrm{A}} d \lambda_{\alpha}^{*}\left(\mathrm{X}_{\alpha}-i \mathrm{Y}_{\alpha}\right) \sum_{\beta \in \mathrm{A}} \lambda_{\beta}\left(\mathrm{X}_{\beta}+i \mathrm{Y}_{\beta}\right) \\
& =\sum_{\alpha, \beta \in \mathrm{A}} \lambda_{\alpha}^{*} d \lambda_{\beta}\left[\mathrm{X}_{\alpha} \mathrm{X}_{\beta}+\mathrm{Y}_{\alpha} \mathrm{Y}_{\beta}+i\left(\mathrm{X}_{\alpha} \mathrm{Y}_{\beta}-\mathrm{Y}_{\alpha} \mathrm{X}_{\beta}\right)\right] \\
& -\sum_{\alpha, \beta \in \mathrm{A}} d \lambda_{\alpha}^{*} \lambda_{\beta}\left[\mathrm{X}_{\alpha} \mathrm{X}_{\beta}+\mathrm{Y}_{\alpha} \mathrm{Y}_{\beta}+i\left(\mathrm{X}_{\alpha} \mathrm{Y}_{\beta}-\mathrm{Y}_{\alpha} \mathrm{X}_{\beta}\right)\right] \\
& =\sum_{\alpha, \beta \in \mathrm{A}}\left(\mathrm{X}_{\alpha} \mathrm{X}_{\beta}+\mathrm{Y}_{\alpha} \mathrm{Y}_{\beta}\right)\left(\lambda_{\alpha}^{*} d \lambda_{\beta}-d \lambda_{\alpha}^{*} \lambda_{\beta}\right) \\
& +i \sum_{\alpha, \beta \in \mathrm{A}}\left(\mathrm{X}_{\alpha} \mathrm{Y}_{\beta}-\mathrm{Y}_{\alpha} \mathrm{X}_{\beta}\right)\left(\lambda_{\alpha}^{*} d \lambda_{\beta}-d \lambda_{\alpha}^{*} \lambda_{\beta}\right) .
\end{aligned}
$$

But, $\sum_{\alpha, \beta \in \mathrm{A}}\left(\mathrm{X}_{\alpha} \mathrm{X}_{\beta}+\mathrm{Y}_{\alpha} \mathrm{Y}_{\beta}\right)\left(\lambda_{\alpha}^{*} d \lambda_{\beta}-d \lambda_{\alpha}^{*} \lambda_{\beta}\right)=0$ for symmetry reasons. Hence, developing, for each index, $\lambda_{\alpha}=a_{\alpha}+i b_{\alpha}$, we get: 


$$
\begin{aligned}
(\mathrm{Z} \cdot d \mathrm{Z}-d \mathrm{Z} \cdot \mathrm{Z})(\mathrm{P}) & =i \sum_{\alpha, \beta \in \mathrm{A}}\left(\mathrm{X}_{\alpha} \mathrm{Y}_{\beta}-\mathrm{Y}_{\alpha} \mathrm{X}_{\beta}\right)\left(\lambda_{\alpha}^{*} d \lambda_{\beta}-d \lambda_{\alpha}^{*} \lambda_{\beta}\right) \\
& =i \sum_{\alpha, \beta \in \mathrm{A}}\left(\mathrm{X}_{\alpha} \mathrm{Y}_{\beta}-\mathrm{Y}_{\alpha} \mathrm{X}_{\beta}\right)\left(a_{\alpha} d a_{\beta}-a_{\beta} d a_{\alpha}+b_{\alpha} d b_{\beta}-b_{\beta} d b_{\alpha}\right) \\
& -\sum_{\alpha, \beta \in \mathrm{A}}\left(\mathrm{X}_{\alpha} \mathrm{Y}_{\beta}-\mathrm{Y}_{\alpha} \mathrm{X}_{\beta}\right)\left(a_{\alpha} d b_{\beta}+a_{\beta} d b_{\alpha}-b_{\alpha} d a_{\beta}-b_{\beta} d a_{\alpha}\right) .
\end{aligned}
$$

But, the second term of the right hand side vanishes for symmetry reasons. So, there remains:

$$
\begin{aligned}
(\mathrm{Z} \cdot d \mathrm{Z}-d \mathrm{Z} \cdot \mathrm{Z})(\mathrm{P}) & =i \sum_{\alpha, \beta \in \mathrm{A}}\left(\mathrm{X}_{\alpha} \mathrm{Y}_{\beta}-\mathrm{Y}_{\alpha} \mathrm{X}_{\beta}\right)\left(a_{\alpha} d a_{\beta}-a_{\beta} d a_{\alpha}\right) \\
& +i \sum_{\alpha, \beta \in \mathrm{A}}\left(\mathrm{X}_{\alpha} \mathrm{Y}_{\beta}-\mathrm{Y}_{\alpha} \mathrm{X}_{\beta}\right)\left(b_{\alpha} d b_{\beta}-b_{\beta} d b_{\alpha}\right)
\end{aligned}
$$

Let us now come back to the map $\Phi: \mathrm{Z} \mapsto(\mathrm{X}, \mathrm{Y})$, identifying $\mathcal{H}$ and $\mathcal{H}_{\mathbf{R}} \times \mathcal{H}_{\mathbf{R}}$. The plot $\Phi \circ \mathrm{P}$ writes necessarily $\Phi \circ \mathrm{P}(r)=\sum_{j \in \mathcal{J}} \mu_{j}(r)\left(\mathrm{X}_{j}, \mathrm{Y}_{j}\right)$. Then, by developing $\sum_{\alpha \in \mathrm{A}} \lambda_{\alpha} \mathrm{Z}_{\alpha}$ we obtain the family $\left(\mu_{j},\left(\mathrm{X}_{j}, \mathrm{Y}_{j}\right)\right)_{j \in \mathcal{J}}$ as the union of two families:

$$
\left(\mu_{j},\left(\mathrm{X}_{j}, \mathrm{Y}_{j}\right)\right)_{j \in \mathcal{J}}=\left(a_{\alpha},\left(\mathrm{X}_{\alpha}, \mathrm{Y}_{\alpha}\right)_{\alpha \in \mathrm{A}} \cup b_{\alpha},\left(-\mathrm{Y}_{\alpha}, \mathrm{X}_{\alpha}\right)_{\alpha \in \mathrm{A}}\right)
$$

Applying the form $\varpi$ to $\Phi \circ \mathrm{P}$, with this family, we get:

$$
\begin{aligned}
\varpi(\Phi \circ \mathrm{P}) & =\sum_{\alpha, \beta \in \mathrm{A}}\left(\mathrm{X}_{\alpha} \mathrm{Y}_{\beta}-\mathrm{Y}_{\alpha} \mathrm{X}_{\beta}\right)\left(a_{\alpha} d a_{\beta}-a_{\beta} d a_{\alpha}\right) \\
& +\sum_{\alpha, \beta \in \mathrm{A}}\left(-\mathrm{Y}_{\alpha} \mathrm{X}_{\beta}+\mathrm{X}_{\alpha} \mathrm{Y}_{\beta}\right)\left(b_{\alpha} d b_{\beta}-b_{\beta} d b_{\alpha}\right) .
\end{aligned}
$$

Comparing the last two expressions we get $\varpi(\Phi \circ \mathrm{P})=(1 / 2 i)(\mathrm{Z} \cdot d \mathrm{Z}-d \mathrm{Z} \cdot \mathrm{Z})(\mathrm{P})$. Thanks to locality (art. 10.4), this equality is still satisfied for any plot of $\mathcal{H}_{\mathbf{R}}^{2}$. Hence, we can conclude that:

$$
\Phi^{*}(\varpi)=\frac{1}{2 i}[\mathrm{Z} \cdot d \mathrm{Z}-d \mathrm{Z} \cdot \mathrm{Z}]
$$

The proof is complete.

20. The symplectic form on the Hilbert square. The standard symplectic form of $\mathcal{H}_{\mathbf{R}} \times \mathcal{H}_{\mathbf{R}}$ is just the exterior derivative of the Liouville form. It generalizes the standard symplectic form of even dimensional real vector spaces, and its local expression is given by the following proposition.

20.1. The symplectic form of $\mathcal{H}_{\mathbf{R}} \times \mathcal{H}_{\mathbf{R}}$. Let $\Omega$ be the exterior derivative of $\Lambda$,

$$
\Omega=d \Lambda, \quad \text { i.e. } \quad \Omega(\mathrm{P})=d[\Lambda(\mathrm{P})]
$$

for any plot $\mathrm{P}: \mathrm{U} \longrightarrow \mathcal{H}_{\mathbf{R}} \times \mathcal{H}_{\mathbf{R}}$. Let $\mathrm{V} \subset \mathrm{U}$ be a domain such that there exists a local family $\left(\lambda_{\alpha},\left(\mathrm{X}_{\alpha}, \mathrm{Y}_{\alpha}\right)\right)_{\alpha \in \mathrm{A}}$ such that:

$$
\mathrm{P} \uparrow \mathrm{V}: r \mapsto \sum_{\alpha \in \mathrm{A}} \lambda_{\alpha}(r)\left(\mathrm{X}_{\alpha}, \mathrm{Y}_{\alpha}\right) .
$$


Then, a direct computation give the local expresion of the 2-form $\Omega$ in the plot P:

$$
\Omega(\mathrm{P} \uparrow \mathrm{V})=\sum_{\alpha, \beta \in \mathrm{A}}\left(\mathrm{X}_{\alpha} \cdot \mathrm{Y}_{\beta}-\mathrm{Y}_{\alpha} \cdot \mathrm{X}_{\beta}\right) d \lambda_{\alpha} \wedge d \lambda_{\beta}
$$

Moreover the symplectic form $\Omega$ is invariant under the translations of $\mathcal{H}_{\mathbf{R}} \times \mathcal{H}_{\mathbf{R}}$. We call $\Omega$ the standard symplectic form of the square $\mathcal{H}_{\mathbf{R}} \times \mathcal{H}_{\mathbf{R}}$.

20.2. Why is $\Omega$ symplectic? The word symplectic in the last proposition (art. 20.1) needs however to be discussed. I use it because the momentum map of $\mathcal{H}_{\mathbf{R}} \times \mathcal{H}_{\mathbf{R}}$, acting on itself by translation and preserving $\omega$, satisfies the condition I suggest for $\omega$ to be called a symplectic form. Here is an abridged description of the general construction, a full discussion on symplectic diffeology can be found in [PIZ05].

Let X be a connected diffeological space. Let $\omega$ be a closed 2-form on X. Its whole symmetry group Diff $(\mathrm{X}, \omega)$ consists of all diffeomorphisms of $\mathrm{X}$ which preserve $\omega$, equipped with the functional diffeology (art. 7.1). Let $\mathrm{G}$ be any group of symmetries, that is any subgroup of $\operatorname{Diff}(\mathrm{X}, \omega)$, and let $\mathcal{G}^{*}$ be the space of moments of $\mathrm{G}$ (art. 11.2) equipped with the functional diffeology of space of differentiable forms [PIZ05].

The 2-point momentum map. The 2-point momentum map of $\mathrm{G}$ is a differentiable map $\psi$ defined on $\mathrm{X} \times \mathrm{X}$ with values in a quotient $\mathcal{G}^{*} / \Gamma$, where $\Gamma$ is the holonomy group of the G-action. The holonomy group $\Gamma$ is a homomorphic image of the first homotopy group $\pi_{1}(\mathrm{X})$, and is G-invariant in $\mathcal{G}^{*}$. It is the obstruction to the G-action being hamiltonian. The 2-point momentum map satisfies the Chasles cocycle relation

$$
\psi\left(x, x^{\prime}\right)+\psi\left(x^{\prime}, x^{\prime \prime}\right)+\psi\left(x^{\prime \prime}, x\right)=0
$$

and is G-equivariant:

$$
\psi\left(g(x), g\left(x^{\prime}\right)\right)=\operatorname{Ad}^{*}(g)\left(\psi\left(x, x^{\prime}\right)\right), \text { for all } g \in \mathrm{G} .
$$

The 1-point momentum maps. Since $\mathrm{X}$ is connected, there always exists a differentiable map $\mu$ from $\mathrm{X}$ to $\mathcal{G}^{*} / \Gamma$ such that

$$
\psi\left(x, x^{\prime}\right)=\mu\left(x^{\prime}\right)-\mu(x) .
$$

We can choose $\mu(x)=\psi\left(x_{0}, x\right)$ where $x_{0}$ is any point of X. I call the maps $\mu$, the 1 -point momentum maps. We may also simply call them momentum maps, since they extend the usual definition of the momentum maps. Since the space $\mathrm{X}$ is connected, two momentum maps differ only by a constant. If $\mu$ can be chosen equivariant the 2 -point momentum map $\psi$ is said to be exact, and $\mu$ is called a primitive of $\psi$.

The associated cohomology class $\theta$. If the 2-point momentum map $\psi$ is not exact, the variance of any 1-momentum map $\mu$ defines a non trivial 1-cocycle $\theta$ by:

$$
\mu(g(x))=\operatorname{Ad}^{*}(g)(\mu(x))+\theta(g), \quad \text { where } \theta \in \mathrm{Z}^{1}\left(\mathrm{G}, \mathcal{G}^{*} / \Gamma\right) .
$$

Two different 1-point momentum maps define two cohomologous cocycles. The cohomology class $[\theta] \in \mathrm{H}^{1}\left(\mathrm{G}, \mathcal{G}^{*} / \Gamma\right)$ extends the so-called Souriau cohomology class. Note that if the 2-point momentum map $\psi$ is exact, a primitive $\mu$ of $\psi$ (that is a 1-point momentum map) is still defined modulo a constant. But, this time this constant is invariant under the coadjoint action of $\mathrm{G}$. 
Homogeneous cases. Whether $\mu$ is equivariant or not, we can show [PIZ05] that when $\mathrm{G}$ acts transitively on $\mathrm{X}, \mu$ is automatically a fibration onto its image which is always an affine coadjoint orbit, that is an orbit of the affine coadjoint action:

$$
g_{\theta}: \mu \mapsto \operatorname{Ad}^{*}(g)(\mu)+\theta(g) \text {, for any } g \in \mathrm{G} \text { and any } \mu \in \mathcal{G}^{*} / \Gamma \text {. }
$$

What has been said until now applies the same way to the whole symmetry group $\operatorname{Diff}(\mathrm{X}, \omega)$. In this case the momentum map is the universal momentum map of $(\mathrm{X}, \omega)$, since any other momentum map, relative to any subgroup $G \subset \operatorname{Diff}(X, \omega)$ factorizes through it. Now, it seems to me natural to define symplectic forms as follow:

Symplectic diffeological spaces. Let X be a diffeological space. A closed 2-form $\omega$ defined on $\mathrm{X}$ is said to be symplectic if $\mathrm{X}$ is homogeneous under the symmetry group $\operatorname{Diff}(\mathrm{X}, \omega)$ and if any 1-point momentum map $\mu$ of $\operatorname{Diff}(X, \omega)$ is a covering onto its image. In this case, the pair $(\mathrm{X}, \omega)$ is called a symplectic diffeological space.

This definition needs however some comments. First of all, if this property is satisfied for some group of symmetries $\mathrm{G} \subset \operatorname{Diff}(\mathrm{X}, \omega)$, it is a fortiori satisfied for the whole group of symmetries $\operatorname{Diff}(X, \omega)$. Hence, it is sufficient to find the good symmetry group for checking that a closed 2-form is symplectic. Secondly, if a 1-point momentum map is a covering onto its image then all the 1-points momentum maps are coverings, since they differ just by a constant.

Now, let us come to the very reason of this definition. In the usual case of a finite dimensional real manifold, the Darboux theorem asserts that a symplectic manifold $(\mathrm{X}, \omega)$ is locally homogeneous under $\operatorname{Diff}(\mathrm{X}, \omega)$. Hence, a good generalization of symplectic manifolds needs to include this property, which excludes the non homogeneous situations, altought this condition could weakened by considering the pseudogroup of local automorphisms, but that is still not done. Then, if $\mathrm{X}$ is homogeneous under $\operatorname{Diff}(\mathrm{X}, \omega)$ or one of its subgroups, any 1-point momentum map $\mu$ is a fibration onto an affine coadjoint orbit. The space $\mathrm{X}$ can be regarded as pre-symplectic. But, the characteristics of $\mu$ are exactly the characteristics of $\omega$. Hence to be non degenerate is equivalent for $\mu$ to have its characteristics discrete, that is to be a covering onto its image.

I know that many authors consider som kinds of "symplectic structures" on non homogeneous spaces, as for example on orbifolds where singularities can be exchanged by diffeomorphisms only with other singularities of same type. Personally, for the reason just expressed above, I would prefer to talk about "symplectic stratified diffeological spaces", althout symplectic stratified diffeological spaces can be more general than orbifolds. But we still miss a name for the general case of a diffeological space equipped just with a closed 2-form, which is the basis of all this analysis.

21. The symplectic structure on the infinite projective space. We consider now the restriction $\varpi$ of the Liouville form $\Lambda$ to the sphere $\mathcal{S}_{\mathbf{R}}$, it is obviously invariant by the action of $\mathrm{SO}(2, \mathbf{R})$. In the complex picture, the action of $\mathrm{S}^{1} \sim \mathrm{SO}(2, \mathbf{R})$ is given by multiplication. For any point $\mathrm{Z} \in \mathcal{S}$, the orbit map $\hat{\mathrm{Z}}$, from $\mathrm{S}^{1}$ to $\mathcal{S}$, is defined by:

$$
\hat{\mathrm{Z}}: \mathrm{S}^{1} \longrightarrow \mathcal{S} \text { with } \hat{\mathrm{Z}}(\tau)=\tau \mathrm{Z} .
$$


21.1. The Liouville form $\varpi$ is a connection form. Let $\varpi$ be the restriction of the Liouville form of $\mathcal{H}_{\mathbf{R}}^{2}$ to the infinite sphere $\mathcal{S}_{\mathbf{R}}$. For any $\mathrm{Z}$ in $\mathcal{S}$, the pull-back of the Liouville form $\varpi$ by the orbit map $\hat{Z}$ coincides with the standard length 1-form of the circle $S^{1}$ :

$$
\hat{\mathrm{Z}}^{*}(\varpi)=\frac{d z}{i z} \text {. }
$$

In other words, $\varpi$ is an $S^{1}$-connection form of the Hopf fibration $\pi: \mathcal{S} \rightarrow \mathcal{P}$.

Proof. It is enough to test the 1-form $\mathrm{Z}^{*}(\varpi)$ on the 1-plots of $\mathrm{S}^{1}$ (art. 10.5). And since the diffeology of $\mathrm{S}^{1}$ is generated by the homomorphism $\mathrm{F}: \mathbf{R} \rightarrow \mathrm{S}^{1}$, with $\mathrm{F}(t)=e^{i t}$, it is enough to test $\mathrm{Z}^{*}(\varpi)$ on F. Now,

$$
\mathrm{F}^{*}\left(\hat{\mathrm{Z}}^{*}(\varpi)\right)=(\hat{\mathrm{Z}} \circ \mathrm{F})^{*} \varpi=\varpi(\hat{\mathrm{Z}} \circ \mathrm{F})=\varpi([t \mapsto \mathrm{F}(t) \mathrm{Z}]) .
$$

Let us develop $\mathrm{F}(t) \mathrm{Z}$ in terms of $(\mathrm{X}, \mathrm{Y})$ :

$$
\mathrm{Z}=\left(\begin{array}{l}
\mathrm{X} \\
\mathrm{Y}
\end{array}\right), \quad \mathrm{F}(t)=\left(\begin{array}{cc}
\cos (t) & -\sin (t) \\
\sin (t) & \cos (t)
\end{array}\right), \quad \mathrm{F}(t)(\mathrm{Z})=\left(\begin{array}{c}
\cos (t) \mathrm{X}-\sin (t) \mathrm{Y} \\
\sin (t) \mathrm{X}+\cos (t) \mathrm{Y}
\end{array}\right)
$$

In other words, our plot $t \mapsto \mathrm{F}(t)(\mathrm{Z})$ can be described in terms of (art. 13.1) the local family $\left(\lambda_{1}, \mathrm{X}_{1}, \mathrm{Y}_{1}\right)=(\cos , \mathrm{X}, \mathrm{Y}),\left(\lambda_{2}, \mathrm{X}_{2}, \mathrm{Y}_{2}\right)=(\sin ,-\mathrm{Y}, \mathrm{X})$.

Now, let us apply the definition,

$$
\begin{aligned}
\varpi([t \mapsto \mathrm{F}(t) \mathrm{Z}])= & \sum_{\alpha, \beta \in\{1,2\}} \mathrm{X}_{\alpha} \mathrm{Y}_{\beta}\left(\lambda_{\alpha} d \lambda_{\beta}-\lambda_{\beta} d \lambda_{\alpha}\right) \\
= & \mathrm{X}_{1} \mathrm{Y}_{2}\left(\lambda_{1} d \lambda_{2}-\lambda_{2} d \lambda_{1}\right)+\mathrm{X}_{2} \mathrm{Y}_{1}\left(\lambda_{2} d \lambda_{1}-\lambda_{1} d \lambda_{2}\right) \\
= & \mathrm{X} \cdot \mathrm{X}[\cos (t) d(\sin (t))-\sin (t) d(\cos (t))] \\
& +(-\mathrm{Y}) \cdot \mathrm{Y}[\sin (t) d(\cos (t))-\cos (t) d(\sin (t))] \\
= & \mathrm{X} \cdot \mathrm{X} d t+\mathrm{Y} \cdot \mathrm{Y} d t \\
= & {[\mathrm{X} \cdot \mathrm{X}+\mathrm{Y} \cdot \mathrm{Y}] d t } \\
= & d t \\
= & \mathrm{F}^{*}(d z / i z)
\end{aligned}
$$

The proof is complete.

21.2. Fubini-Study form on the infinite projective space There exists a unique closed 2 -form $\omega$ defined on $\mathcal{P}$ such that

$$
\pi^{*} \omega=d \varpi, \quad \pi: \mathcal{S} \longrightarrow \mathcal{P} .
$$

It is, by definition, the curvature of $\varpi$, viewed as a $S^{1}$-connection form on $\mathcal{S}$. This form $\omega$ will be called the infinite Fubini-Study symplectic form.

Proof. In general, when we have a subduction $\pi: \mathrm{A} \rightarrow \mathrm{B}$ and a form $\alpha$ on $\mathrm{A}$, for proving that there exists a form $\beta$ on $\mathrm{B}$ such that $\pi^{*}(\beta)=\alpha$, we have to check that: for any pair of plots $\mathrm{P}$ and $\mathrm{P}^{\prime}$ of $\mathrm{A}$ such that $\pi \circ \mathrm{P}=\pi \circ \mathrm{P}^{\prime}, \alpha(\mathrm{P})=\alpha\left(\mathrm{P}^{\prime}\right)$ [PIZ05]. But in the case of principal diffeological fibration this condition splits into two conditions:

a) the form $\alpha$ must be invariant under the structure group, and

b) it must vanish on the "vertical" plots, that is the plots whose images are contained in the fibers. 
The details of these propositions can be found in [PIZ05]. In our case, the fiber has dimension 1 . So, $d \varpi$ necessarily vanishes on the vertical plots, and we noted already that $\varpi$ (therefore $d \varpi)$ is $\mathrm{S}^{1}$-invariant (art. 21.1). Hence, there exists a 2-form $\omega$ of $\mathcal{P}$, such that $d \varpi=\pi^{*} \omega$.

21.3. The infinite Fubini-Study form is not exact. The Fubini-Study form $\omega$ on $\mathcal{P}$ is the curvature of the connection $\varpi$. It is closed but not exact, and its value on any 2-cycle is a multiple of $2 \pi$. In other words, its group of periods is

$$
\operatorname{Per}(\omega)=\left\{\int_{\sigma} \omega \mid \sigma \in \mathrm{H}_{2}(\mathcal{P}, \mathbf{Z})\right\}=2 \pi \mathbf{Z} .
$$

Proof. $\omega$ is not exact. Suppose we had $\omega=d \epsilon$. Then, $\varpi-\pi^{*}(\epsilon)$ would be closed: $d[\varpi-$ $\left.\pi^{*}(\epsilon)\right]=\pi^{*}(\omega-d \epsilon)=0$. Since $\mathcal{S}$ is contractible (art. 17.2), it would follow [PIZ05] that $\varpi-\pi^{*}(\epsilon)$ is exact, say $\varpi-\pi^{*}(\epsilon)=d f$. But then the integral over a fiber $\mathrm{S}=\pi^{-1}(p)$ would vanish: $\int_{\mathrm{S}} \varpi=\int_{\mathrm{S}} \pi^{*}(\epsilon)+\int_{\mathrm{S}} d f=0+\int_{\partial \mathrm{S}} f=0+0$, whereas we know that $\int_{\mathrm{S}} \varpi=\int_{0}^{2 \pi} d t=2 \pi$.

The periods of $\omega$. Let us consider now a 2-simplex $\sigma$ in $\mathcal{P}$, such that $\partial \sigma=0$, i.e. its boundary goes on one point $p$. Let us compute the integral $\int_{\sigma} \omega=\int_{\sigma} \pi_{*}(d \varpi)=$ $\int_{\pi^{*} \sigma} d \varpi$. Here, $\pi^{*} \sigma$ is a lifting $\sigma^{*}$ of $\sigma$, that is a simplex of $\mathcal{S}$ such that $\pi \circ \sigma^{*}=\sigma$. So, $\int_{\sigma} \omega=\int_{\partial \sigma^{*}} \varpi$, but $\partial \sigma^{*}$ is a map sending a « circle» - the boundary of the standard 2 -simplex — to the circle $\pi^{-1}(p)$. Since $\varpi \uparrow \pi^{-1}(p) \sim d t$, by definition of the degree: $\int_{\partial \sigma^{*}} \varpi=\operatorname{deg}\left(\partial \sigma^{*}\right) \int_{0}^{2 \pi} d t \in 2 \pi \mathbf{Z}$.

22. The canonical line bundle over the infinite projective space. It is difficult to talk about the Hopf fibration without talking about its $\mathbf{C}$-associated line bundle.

22.1. The Hopf line bundle. Let us consider the diagonal action of $\mathbf{C}^{\star}$ on the product $\mathcal{H}^{\star} \times \mathbf{C}$, endowed with the product diffeology. It is easy to check that this action is differentiable. Now, the quotient space:

$$
\mathcal{L}=\mathcal{H}^{\star} \times_{\mathbf{C}^{\star}} \mathbf{C}=\left\{[\mathrm{Z}, z] \mid(\mathrm{Z}, z) \sim(\tau \mathrm{Z}, \tau z), \tau \in \mathbf{C}^{\star}\right\}
$$

equipped with its quotient topology, is a diffeological bundle, on $\mathcal{P}$, locally trivial. The projection is defined by $\pi:[\mathrm{Z}, z] \mapsto[\mathrm{Z}]$. Moreover, $\mathcal{L}$ is a diffeological manifold modeled on $\mathcal{H}$.

Proof. Let us consider the following maps $\Phi_{k}$ :

$$
\Phi_{k}: \mathcal{H} \times \mathbf{C} \rightarrow \mathcal{L} \text { such that } \Phi_{k}(\mathrm{Z}, z)=\left[\mathrm{F}_{k}(\mathrm{Z}), z\right] \in \mathcal{L},
$$

where $\mathrm{F}_{k}$ are the charts defined in proposition 18.1. The $\Phi_{k}$ are diffeomorphisms which commute with the action of $\mathbf{C}^{\star}$, therefore $\mathcal{L}$ is locally diffeomorphic to $\mathcal{H} \times \mathbf{C} \sim \mathcal{H}$ at each point. The set of $\left(\Phi_{k}\right)_{k \in \mathbf{N}^{*}}$ is an equivariant atlas of $\mathcal{L}$.

22.2. Sections of the line bundle. Note that, by restriction to $\mathcal{S} \subset \mathcal{H}^{\star}$ and $\mathrm{S}^{1} \subset \mathbf{C}^{\star}$, we get:

$$
\mathcal{L}=\mathcal{S} \times_{\mathrm{S}^{1}} \mathbf{C}=\left\{[\mathrm{Z}, z] \mid(\mathrm{Z}, z) \sim(\tau \mathrm{Z}, \tau z), \tau \in \mathrm{S}^{1}\right\} .
$$

Outside the null-section, the line bundle $\mathcal{L}$ is diffeomorphic to $\mathcal{H}^{\star}$, the point $0 \in \mathcal{H}$ is replaced by $\mathcal{P}$. The space $\mathcal{L}$ is the blow-up of $\mathcal{H}$ at the point 0 . 
Now, let $\psi: \mathcal{S} \rightarrow \mathbf{C}$ be a differentiable function such that $\psi(\tau \mathrm{Z})=\tau \psi(\mathrm{Z})$, for all $\tau \in \mathrm{S}^{1}$ and $\mathrm{Z} \in \mathcal{S}$. The map $[\psi]$ defined by:

$$
[\psi]: \mathcal{P} \rightarrow \mathcal{L} \quad \text { such that }[\psi]([\mathrm{Z}])=[\mathrm{Z}, \psi(\mathrm{Z})]
$$

is a differentiable section of the bundle $\pi: \mathcal{L} \rightarrow \mathcal{P}$.

Proof. The proof is an application of general constructions about associated diffeological fiber bundles, developed in [Igl85].

\section{THE SYMMETRIES OF THE INFINITE HOPF CONSTRUCTION}

We used the word symplectic to describe the 2-form $\omega$ defined on the infinite projective space $\mathcal{P}$ (art. 21.2). But we have to justify this terminology. According to the suggestion above (art. 20.2) we shall show that the projective space is equivalent to a coadjoint orbit of the unitary group $\mathbf{U}(\mathcal{H})$, that is the subgroup of $\mathrm{GL}(\mathcal{H})$ preserving the hermitian form. First of all we shall show that $\mathcal{P}$ is homogeneous under $\mathbf{U}(\mathcal{H})$, for the diffeologies involved. Then, we compute explicitly the momentum map of the action of $\mathbf{U}(\mathcal{H})$ on $\mathcal{P}$ and show that it is bijective. This will identify $\mathcal{P}$ with the image of the momentum map, that is with a coadjoint orbit of $\mathbf{U}(\mathcal{H})$.

23. The infinite sphere as a homogeneous space. On one hand, the vector space $\mathcal{H}$ is equipped with its fine diffeology, the sphere $\mathcal{S} \subset \mathcal{H}$ inherits its diffeology by induction. On the other hand, the space of linear maps $\mathrm{L}(\mathcal{H})$ is equipped with the functional diffeology, as group of differentiable maps of $\mathcal{H}$, the subgroup $\mathbf{U}(\mathcal{H}) \subset \mathrm{L}(\mathcal{H})$ inherits, by induction, this functional diffeology described in (art. 13.7). The group $\mathbf{U}(\mathcal{H})$ acts transitively on the sphere $\mathcal{S}$ [Bou72]. We shall show that $\mathcal{S}$ is a homogeneous space of $\mathbf{U}(\mathcal{H})$. That is, precisely: the diffeology of the sphere $\mathcal{S}$ is the quotient of the functional diffeology of $\mathbf{U}(\mathcal{H})$.

23.1. The infinite sphere as homogeneous space. Let $\pi: \mathbf{U}(\mathcal{H}) \longrightarrow \mathcal{S}$ be the map $\pi(\mathrm{A})=$ $\mathrm{Ae}_{1}$. The map $\pi$ is a subduction, where $\mathbf{U}(\mathcal{H})$ is equipped with the functional diffeology and the sphere $\mathcal{S}$ with the subset diffeology of the fine diffeology of $\mathcal{H}$. In other words, the sphere $\mathcal{S}$ is a diffeological homogeneous space of $\mathbf{U}(\mathcal{H})$.

$$
\mathcal{S} \simeq \mathbf{U}(\mathcal{H}) / \operatorname{St}_{\mathbf{U}(\mathcal{H})}\left(\mathbf{e}_{1}\right) \text { and } \operatorname{St}_{\mathbf{U}(\mathcal{H})}\left(\mathbf{e}_{1}\right) \simeq \mathbf{U}(\mathcal{H})
$$

Proof. The proof will be given in two steps:

1. The map $\pi$ is surjective. Let $\mathrm{Z}$ and $\mathrm{Z}^{\prime}$ be two elements of $\mathcal{S}$. If $\mathrm{Z}$ and $\mathrm{Z}^{\prime}$ are collinear then there exists $\tau \in \mathrm{S}^{1} \simeq \mathbf{U}(\mathbf{C})$ such that $\mathrm{Z}^{\prime}=\tau \mathrm{Z}$. But, the map $\mathrm{Z} \mapsto \tau \mathrm{Z}$ belongs to $\mathbf{U}(\mathcal{H})$. Now, if $\mathrm{Z}$ and $\mathrm{Z}^{\prime}$ are independent over $\mathbf{C}$, let $\mathrm{E}$ be the plane spanned by these two vectors and let $\mathrm{F}$ be its orthogonal for the hermitian product. According to Bourbaki [Bou72] $\mathrm{E}$ and $\mathrm{F}$ are supplementary $\mathcal{H}=\mathrm{E} \oplus \mathrm{F}$. The vectors $\mathrm{Z}$ and $\mathrm{Z}^{\prime}$ are vectors of the unit sphere $\mathrm{S}^{3} \subset \mathrm{E} \simeq \mathbf{C}^{2}$, now the group $\mathbf{U}\left(\mathbf{C}^{2}\right)$ acts transitively on $\mathrm{S}^{3}$, there exists $\mathrm{A} \in \mathbf{U}\left(\mathbf{C}^{3}\right)$ such that $\mathrm{Z}^{\prime}=\mathrm{AZ}$. This map, extended to $\mathcal{H}$ by the identity on $\mathrm{F}$, belongs to $\mathbf{U}(\mathcal{H})$ and maps $\mathrm{Z}$ to $\mathrm{Z}^{\prime}$. Therefore, the action of $\mathbf{U}(\mathcal{H})$ is transitive on $\mathcal{S}$, which is equivalent to the assertion that $\pi$ is surjective. 
2. The map $\pi$ is a subduction. Let $\mathrm{Q}: \mathrm{U} \longrightarrow \mathcal{S}$ be a plot. We want to lift locally $\mathrm{Q}$ along the projection $\pi$, that is for any $r_{0} \in \mathrm{U}$, find a plot $\mathrm{P}: \mathrm{V} \rightarrow \mathrm{U}(\mathcal{H})$, defined on some superset $\mathrm{V}$ of $r_{0}$, such that $\mathrm{P}(r)\left(\mathbf{e}_{1}\right)=\mathrm{Q}(r)$, for any $r \in \mathrm{V}$. So, let $r_{0} \in \mathrm{U}$, let $\mathrm{V}$ be a superset of $r_{0}$, let $j: \mathbf{C}^{m} \longrightarrow \mathcal{H}$ be an injection, and let $\phi: \mathrm{V} \rightarrow \mathbf{C}^{m}$ be a smooth parametrization such that $\mathrm{Q} \uparrow \mathrm{V}=j \circ \phi$. Let us denote $\mathrm{E}=j\left(\mathbf{C}^{m}\right)$. The plot $\mathrm{Q}$ of $\mathcal{S}$ takes its values in $\mathrm{E}$, and hence in the unit sphere of $\mathrm{E}: \mathrm{S}(\mathrm{E})=\mathrm{E} \cap \mathcal{S}$. The diffeology induced on $\mathrm{S}(\mathrm{E})$ is the standard diffeology: $\mathrm{S}(\mathrm{E}) \simeq \mathrm{S}^{2 m-1}$. Thus $\mathrm{Q} \uparrow \mathrm{V}$ is an ordinary differentiable map from $\mathrm{V}$ into $\mathrm{S}(\mathrm{E})$. But, we know that the projection from $\mathbf{U}(m)$ onto $\mathrm{S}\left(\mathbf{C}^{m}\right)$ is a submersion, a fortiori a subduction. So, for any $r_{0} \in \mathrm{V}$ there exists a domain $\mathrm{W} \subset \mathrm{V}$ and a smooth lifting $\varphi: \mathrm{W} \rightarrow \mathbf{U}(m)$ such that $\mathrm{Q}(r)=\varphi(r)\left(\mathbf{e}_{1}^{m}\right)$, for any $r \in \mathrm{W}$, where $\mathbf{e}_{1}^{m}$ is the vector $(1,0, \ldots, 0) \in \mathbf{C}^{m}$. Let us assume that $\mathbf{e}_{1}=j\left(\mathbf{e}_{1}^{m}\right)$, if it is not the case we conjugate everything with some well chosen linear map. Now, let $\mathrm{F}$ be the orthogonal of $\mathrm{E}$. The space $\mathcal{H}$ is the direct sum of $\mathrm{E}$ and $\mathrm{F}$, i.e. $\mathcal{H}=\mathrm{E} \oplus \mathrm{F}$ [Bou72]. Any vector $\mathrm{Z} \in \mathcal{H}$ has a unique decomposition $\mathrm{Z}=\mathrm{Z}_{\mathrm{E}}+\mathrm{Z}_{\mathrm{F}}$ such that $\mathrm{Z}_{\mathrm{E}} \in \mathrm{E}$ and $\mathrm{Z}_{\mathrm{F}} \in \mathrm{F}$. Let us define the following map:

$$
\text { for all } r \in \mathrm{W} \text {, for all } \mathrm{Z} \in \mathcal{H}: \mathrm{P}(r)(\mathrm{Z})=\varphi(r)\left(\mathrm{Z}_{\mathrm{E}}\right)+\mathrm{Z}_{\mathrm{F}} \text {. }
$$

For any $r \in \mathrm{W}$ the map $\mathrm{P}(r)$ is differentiable because the decomposition $\mathrm{Z} \mapsto\left(\mathrm{Z}_{\mathrm{E}}, \mathrm{Z}_{\mathrm{F}}\right)$ is linear, differentiable for the fine diffeology. Moreover $\mathrm{P}(r)$ clearly preserves the Hermitian product, and it's clearly invertible. The map P lifts Q locally:

$$
\text { for all } r \in \mathrm{W}, \quad \mathrm{P}(r)\left(\mathbf{e}_{1}\right)=\varphi(r)\left(\mathbf{e}_{1}^{m}\right)+0=\mathrm{Q}(r) \text {. }
$$

It remains to check that $\mathrm{P}$ is a plot of the functional diffeology of $\mathbf{U}(\mathcal{H})$. But this is quite clear, a finite family of vectors splits into components in $\mathrm{E}$ and in $\mathrm{F}$, because the family is finite one has a finite intersection of open sets which is open and we get the desired property. The inverse of $\mathrm{P}(r)$ does not give any more problem. Thus, the proof is complete.

24. The projective space as a coadjoint orbit. In this section we give first a definition of the momentum map of the action of a diffeological group preserving a closed 2 -form, in the special case where the form is exact and the group preserves a primitive. The general definition of the momentum map in diffeology can be found in [PIZ05]. We apply this definition to the action of $\mathbf{U}(\mathcal{H})$ acting on the infinite sphere equipped with the form $d \varpi$. Then, we show that the momentum map factorizes through $\mathcal{P}$ in a bijection onto a coadjoint orbit of $\mathbf{U}(\mathcal{H})$. Thanks to the equivariance of the momentum map, this identifies $\mathcal{P}$ to a coadjoint orbit.

24.1. The momentum map of the unitary group. Let us consider the action of $\mathbf{U}(\mathcal{H})$ on $\mathcal{S}$, let us denote $A_{\mathcal{S}}: \mathrm{Z} \mapsto \mathrm{AZ}$, where $(\mathrm{A}, \mathrm{Z}) \in \mathbf{U}(\mathcal{H}) \times \mathcal{S}$. This action preserves the Liouville form $\varpi$, that is for all $A \in \mathbf{U}(\mathcal{H}), A_{\mathcal{S}}^{*}(\varpi)=\varpi$. The momentum map associated to the action of $\mathbf{U}(\mathcal{H})$ on $\mathcal{S}$, regarding $d \varpi$ is, by definition the map

$$
\mu: \mathrm{Z} \mapsto \hat{\mathrm{Z}}^{*}(\varpi), \quad \hat{\mathrm{Z}}: \mathbf{U}(\mathcal{H}) \longrightarrow \mathcal{S}, \quad \hat{\mathrm{Z}}(\mathrm{A})=\mathrm{AZ} .
$$

In fact the momentum map is defined modulo a constant (art. 20.2). We make implicitly for $\mu$ and $m$ a choice of constant. 
1. The momentum map $\mu$ takes its values in the space $\mathcal{U}^{*}$ of moments of $\mathbf{U}(\mathcal{H})$ (art. 11.2).

2. The momentum map $\mu$ is equivariant under the action of $\mathbf{U}(\mathcal{H})$ :

For all $\mathrm{A} \in \mathbf{U}(\mathcal{H})$, for all $\mathrm{Z} \in \mathcal{H}, \mu(\mathrm{AZ})=\mathrm{Ad}^{*}(\mathrm{~A})(\mu(\mathrm{Z}))$.

3. The value of the momentum map $\mu$, on any 1-plot $\mathrm{P}$ of $\mathbf{U}(\mathcal{H})$, is given by

$$
\mu(\mathrm{Z})(\mathrm{P})=\frac{1}{2 i}\left[P(t)(\mathrm{Z}) \cdot \frac{d \mathrm{P}(t)(\mathrm{Z})}{d t}-\frac{d \mathrm{P}(t)(\mathrm{Z})}{d t} \cdot P(t)(\mathrm{Z})\right] d t,
$$

where, locally:

$$
\mathrm{P}(t)(\mathrm{Z})=\sum_{\alpha \in \mathrm{A}} \lambda_{\alpha}(t) \mathrm{Z}_{\alpha}, \quad \text { and } \quad d \mathrm{P}(t)(\mathrm{Z})=\sum_{\alpha \in \mathrm{A}} \frac{d \lambda_{\alpha}(t)}{d t} \mathrm{Z}_{\alpha} .
$$

4. The momentum map $\mu$ is homogeneous of degree two:

For all $\mathrm{Z} \in \mathbf{U}(\mathcal{H})$, for all $z \in \mathbf{C}, \mu(z \mathrm{Z})=|z|^{2} \mu(\mathrm{Z})$.

5. The momentum map $\mu$ factorizes through $\mathcal{P}$ : there exists $m \in \mathcal{C}^{\infty}\left(\mathcal{P}, \mathcal{U}^{*}\right)$ such that $\mu=m \circ \pi$, where $\pi$ is the projection from $\mathcal{S}$ onto its quotient $\mathcal{P}$.

6. The map $m: \mathcal{P} \longrightarrow \mathcal{U}^{*}$ is the momentum map of $\mathbf{U}(\mathcal{H})$ acting on $\mathcal{S}$, relative to the Fubini-Study form $\omega$.

7. The momentum map $m: \mathcal{P} \longrightarrow \mathcal{U}^{*}$ is injective.

Therefore, the image of $\mathcal{P}$ under the momentum map $m$ is a coadjoint orbit of $\mathbf{U}(\mathcal{H})$, the momentum map $m$ identifies, as diffeological spaces, $\mathcal{P}$ and this coadjoint orbit.

Proof. Let us prove successively:

1. $\mu(\mathrm{Z})$ is a moment of $\mathbf{U}(\mathcal{H})$. The 1 -form $\varpi$, defined on $\mathcal{S}$ is invariant by the action of the unitary group $\mathrm{U}(\mathcal{H})$, hence for any $\mathrm{A} \in \mathrm{U}(\mathcal{H}), \mathrm{L}(\mathrm{A})^{*}(\mu(\mathrm{Z}))=\mathrm{L}(\mathrm{A})^{*} \circ \hat{\mathrm{Z}}^{*}(\varpi)=$ $(\hat{\mathrm{Z}} \circ \mathrm{L}(\mathrm{A}))^{*}(\varpi)$, but $\hat{\mathrm{Z}} \circ \mathrm{L}(\mathrm{A})=\mathrm{A}_{\mathcal{S}} \circ \hat{\mathrm{Z}}$, hence $\mathrm{L}(\mathrm{A})^{*}(\mu(\mathrm{Z}))=(\hat{\mathrm{Z}} \circ \mathrm{L}(\mathrm{A}))^{*}(\varpi)=\hat{\mathrm{Z}}^{*}\left(\mathrm{~A}_{\mathcal{S}}^{*}(\varpi)\right)=$ $\hat{\mathrm{Z}}^{*}(\varpi)=\mu(\mathrm{Z})$. Therefore $\mu(\mathrm{Z}) \in \mathcal{U}^{*}$ for any $\mathrm{Z} \in \mathcal{S}$.

2. The momentum map $\mu$ is equivariant. Let $\mathrm{A} \in \mathbf{U}(\mathcal{H})$, and $\mathrm{Z} \in \mathcal{H}$. We have $\mu(\mathrm{AZ})=$ $\widehat{\mathrm{AZ}}^{*} \varpi$, but $\widehat{\mathrm{AZ}}=\mathrm{Z} \circ \mathrm{R}(\mathrm{A})$, so $\mu(\mathrm{AZ})=(\mathrm{Z} \circ \mathrm{R}(\mathrm{A}))^{*}(\varpi)=\mathrm{R}(\mathrm{A})^{*}\left(\hat{\mathrm{Z}}^{*}(\varpi)\right)=\mathrm{R}(\mathrm{A})^{*}(\mu(\mathrm{Z}))=$ $\operatorname{Ad}^{*}(\mathrm{~A}) \circ \mu(\mathrm{Z})$. That is $\mu \circ \mathrm{A}_{\mathcal{S}}=\operatorname{Ad}^{*}(\mathrm{~A}) \circ \mu$.

3. Expression of $\mu$. This expression is a direct application of the complex formulation of the momentum map (art. 19.2).

4. The momentum map is quadratic. It's just an application of the formula $\mathbf{c}$. But we can get this property directly. Let $z \in \mathbf{C}$ and $\mathrm{Z} \in \mathcal{H}$, we have $\mu(z \mathrm{Z})=\widehat{z \mathrm{Z}}^{*}(\varpi)=$ $(z \hat{\mathrm{Z}})^{*}(\varpi)=\hat{\mathrm{Z}}^{*}\left(z^{*}(\varpi)\right)$. Now, let us use the complex expression of the Liouville form (art. 19.2),

$$
\varpi=\frac{1}{2 i}[\mathrm{Z} \cdot d \mathrm{Z}-d \mathrm{Z} \cdot \mathrm{Z}]
$$

By definition of the symbol $d \mathrm{Z}$, we get obviously $d(z \mathrm{Z})=z d \mathrm{Z}$. And, thus $z^{*}(\varpi)=z^{*} z \varpi=$ $|z|^{2} \varpi$, that is $\mu(z \mathrm{Z})=|z|^{2} \mu(\mathrm{Z})$.

5. The momentum map factorizes through $\mathcal{P}$. This is a direct consequence of $\mathcal{P} \simeq \mathcal{S} / \mathrm{S}^{1}$ and part of the proposition 4) with $|z|=1$.

6. The map $m$ is the momentum map of $\mathbf{U}(\mathcal{H})$ on $\mathcal{P}$. This proposition makes reference to the general definition of the momentum map for diffeological spaces [PIZ05]. The 
functoriality of the momentum map expresses that: if a group $\mathrm{G}$ has an hamiltonian action on two spaces $(\mathrm{X}, \omega)$ and $\left(\mathrm{X}^{\prime}, \omega^{\prime}\right)$ such that $\pi: X \rightarrow \mathrm{X}^{\prime}$ is a subduction commuting with the two actions of $\mathrm{G}$, so the respective momentum maps $\mu$ and $\mu^{\prime}$ can be chosen such that $\mu^{\prime}=\pi \circ \mu$.

7. The momentum map $m$ is injective. Let us consider $\mathrm{Z}$ and $\mathrm{Z}^{\prime}$ two elements of $\mathcal{S}$ such that $\mu(Z)=\mu\left(Z^{\prime}\right)$. If $Z$ and $Z^{\prime}$ are collinear, then $Z^{\prime}=\tau Z$ and $[Z]=\left[Z^{\prime}\right]$, where $[Z]$ is the class of $\mathrm{Z}$ in $\mathcal{P}$. Then, let us assume that $\mathrm{Z}$ and $\mathrm{Z}^{\prime}$ are not collinear. Let $\mathrm{E}$ be the complex 2-plane generated by $\mathrm{Z}$ and $\mathrm{Z}^{\prime}$. Let us consider an orthonormal basis of $\mathrm{E}$ made with $\mathrm{Z}$ as first vector and $v$ as second, and let $\mathrm{Z}^{\prime}=a \mathrm{Z}+b v$. We have $a^{*} a+b^{*} b=1$ and $b \neq 0$. Let us now consider the plot $\mathrm{P}: \mathbf{R} \longrightarrow \mathbf{U}(\mathcal{H})$ defined as follows:

$$
\mathrm{P}(t)(\mathrm{Z})=\mathrm{Z}, \mathrm{P}(t)(v)=e^{i t} v \text { and } \mathrm{P}(t) \uparrow \mathrm{E}^{\perp}=\mathbf{1} .
$$

The plot $t \mapsto \mathrm{P}(t)(\mathrm{Z})$ decomposes according to the family $\{(1, \mathrm{Z})\}$, applying the formula \&. we get $\mu(\mathrm{Z})(\mathrm{P})=0$. But, the plot $t \mapsto \mathrm{P}(t)\left(\mathrm{Z}^{\prime}\right)$ decomposes according to the family $\{(a, \mathrm{Z}),(b \exp (i t), v)\}$, and

$$
\frac{d \mathrm{P}(t)\left(\mathrm{Z}^{\prime}\right)}{d t}=i b e^{i t} v
$$

Applying we get now $\mu\left(\mathrm{Z}^{\prime}\right)(\mathrm{P})=b^{*} b$. But then, $\mu\left(\mathrm{Z}^{\prime}\right)(\mathrm{P})=\mu(\mathrm{Z})(\mathrm{P})=0$ implies $b=0$, which is in contradiction with the hypothesis $b \neq 0$. Therefore, $\mu(\mathrm{Z})=\mu\left(\mathrm{Z}^{\prime}\right)$ if and only if $\mathrm{Z}^{\prime}=\tau \mathrm{Z}$, with $\tau \in \mathrm{S}^{1}$.

8. The infinite projective space $\mathcal{P}$ is equivalent to a coadjoint orbit of $\mathbf{U}(\mathcal{H})$. Since the projection from $\mathbf{U}(\mathcal{H})$ onto $\mathcal{S}$ is a subduction, and $\mathcal{P}$ is the quotient of $\mathcal{S}$, the projection from $\mathbf{U}(\mathcal{H})$ onto $\mathcal{P}$ is a subduction (art. 5.5). Now, by definition, the diffeology of the coadjoint orbit $\mathcal{O}=m(\mathcal{P})$ is the quotient diffeology of $\mathbf{U}(\mathcal{H})$ by the stabilizer of some point (art. 11.3). Hence, the momentum map $m$ is differentiable, as well as its inverse, that is $m$ is a diffeomorphism from $\mathcal{P}$ onto $\mathcal{O}$.

25. The action of the maximal torus. We denote by $\mathbf{T}(\mathcal{H})$ the subgroup of $\mathbf{U}(\mathcal{H})$ defined by:

$$
\tau:\left(Z_{k}\right)_{k=1}^{\infty} \mapsto\left(\tau_{k} Z_{k}\right)_{k=1}^{\infty} \text { with } \tau=\left(\tau_{k}\right)_{k=1}^{\infty} \text {, and } \tau_{k} \in \mathrm{S}^{1} \text { for all } k .
$$

We call $\mathbf{T}(\mathcal{H})$ the standard maximal torus $\left({ }^{1}\right)$ of $\mathbf{U}(\mathcal{H})$. As a group, $\mathbf{T}(\mathcal{H})$ is isomorphic to the infinite product of circles $\prod_{k=1}^{\infty} \mathrm{S}^{1}$, indexed by the integers, with pointwise multiplication. But its diffeology inherits the diffeology described in (art. 13.7). We shall see now that, here again, diffeology can give a formal status to what is expected about the momentum map of this group.

25.1. The momentum map of the maximal torus. The momentum map of the maximal torus $\mathbf{T}(\mathcal{H})$ is the restriction to $\mathbf{T}(\mathcal{H})$ of the momentum map of the group $\mathbf{U}(\mathcal{H})$. More precisely, let us consider the orbit map of a point $\mathrm{Z} \in \mathcal{S}$ relative to $\mathbf{T}(\mathcal{H})$ :

$$
\hat{\mathrm{Z}}: \mathbf{T}(\mathcal{H}) \longrightarrow \mathcal{S}, \quad \forall \tau \in \mathbf{T}(\mathcal{H}) \quad \hat{\mathrm{Z}}(\tau)=\tau(\mathrm{Z}) .
$$

\footnotetext{
${ }^{1}$ Here, it is just a name.
} 
The pull-back by $\hat{Z}$ of the 1 -form $\varpi$, defined on $\mathcal{S}$, is a $\mathbf{T}(\mathcal{H})$ invariant 1 -form on $\mathbf{T}(\mathcal{H})$, that is an element of the space of moments $\mathcal{T}^{*}$. The map

$$
\mu: \mathcal{S} \longrightarrow \mathcal{T}^{*} \text { such that } \mu(\mathrm{Z})=\hat{\mathrm{Z}}^{*}(\varpi),
$$

is the momentum map of $\mathbf{T}(\mathcal{H})$ on $\mathcal{S}$. The factorization of $\mu$ on $\mathcal{P}$ is the momentum map of $\mathbf{T}(\mathcal{H})$ on $\mathcal{P}$ for the Fubini-Study form $\omega$ :

$$
m: \mathcal{P} \longrightarrow \mathcal{T}^{*}, \quad m([\mathrm{Z}])=\mu(\mathrm{Z}) .
$$

The momentum map $m$ is given by the following formula:

$$
m([\mathrm{Z}])=\sum_{k=1}^{\infty}\left|\mathrm{Z}_{k}\right|^{2} \operatorname{pr}_{k}^{*}(\varepsilon)
$$

where $\varepsilon$ is the standard length form on $\mathrm{S}^{1}$. More precisely, for any plot $\mathrm{P}: \mathrm{U} \longrightarrow \mathbf{T}(\mathcal{H})$, $\mathrm{U} \in \mathbf{R}^{m}, m(\mathrm{Z})$ writes:

$$
m([\mathrm{Z}])(\mathrm{P})=\sum_{k=1}^{\infty}\left|\mathrm{Z}_{k}\right|^{2} \mathrm{P}_{k}^{*}(\varepsilon) \quad \text { with } \quad \mathrm{P}_{k}=\mathrm{pr}_{k} \circ \mathrm{P} .
$$

Note that for any $r \in \mathrm{U}$, any vector $\delta r \in \mathbf{R}^{m}$, the sequence $\mathrm{N} \mapsto \sum_{k=1}^{\mathrm{N}}\left|\mathrm{Z}_{k}\right|^{2} \mathrm{P}_{k}^{*}(\varepsilon)_{r}(\delta r)$ converges, which gives a meaning to this formula.

Proof. Let us recall the expression of $\varpi$ given in (art. 19.2),

$$
\varpi=\frac{1}{2 i}[\mathrm{Z} \cdot d \mathrm{Z}-d \mathrm{Z} \cdot \mathrm{Z}] \quad \text { with } \quad d \mathrm{Z}(\mathrm{P})(r)=\sum_{\alpha \in \mathrm{A}} d \lambda_{\alpha}(r) \mathrm{Z}_{\alpha} .
$$

Hence,

$$
\begin{aligned}
\hat{\mathrm{Z}}^{*} \varpi & =\frac{1}{2 i}[(\tau \mathrm{Z} \cdot d(\tau \mathrm{Z})-d(\tau \mathrm{Z}) \cdot \tau \mathrm{Z}] \\
& =\frac{1}{2 i}\left[\mathrm{Z} \cdot\left(\tau^{*} d \tau \mathrm{Z}\right)-\mathrm{Z} \cdot\left(\tau d \tau^{*} \mathrm{Z}\right)\right] \\
& =\frac{1}{2 i}\left[\mathrm{Z} \cdot\left(\tau^{*} d \tau-\tau d \tau^{*}\right)(\mathrm{Z})\right] \\
& =\frac{1}{2 i} \sum_{k=1}^{\infty}\left|\mathrm{Z}_{k}\right|^{2}\left(\tau_{k}^{*} d \tau_{k}-\tau_{k} d \tau_{k}^{*}\right)
\end{aligned}
$$

But, $\tau_{k} \in \mathrm{S}^{1}$, so $\tau_{k}^{*} d \tau_{k}=-\tau_{k} d \tau_{k}^{*}$. Hence:

$$
\frac{1}{2 i}\left[\tau_{k}^{*} d \tau_{k}-\tau_{k} d \tau_{k}^{*}\right]=\frac{1}{i} \tau_{k}^{*} d \tau_{k}=\tau_{k}^{*}(\varepsilon)
$$

and therefore

$$
\mu(\mathrm{Z})=\hat{\mathrm{Z}}^{*} \varpi=\sum_{k=1}^{\infty}\left|\mathrm{Z}_{k}\right|^{2} \tau_{k}^{*}(\varepsilon) .
$$

To avoid a misinterpretation of the last equality, due to the infinite sum, let us specify its meaning. Let $\mathrm{P}: \mathrm{U} \rightarrow \mathbf{T}(\mathcal{H})$ be a plot. Let $\mathrm{P}(r)=\sum_{\alpha \in \mathrm{A}} \lambda_{\alpha}(r) \mathrm{Z}_{\alpha}$ be a local expression of $\mathrm{P}$, on $\mathrm{V} \subset \mathrm{U}$. The $\lambda_{\alpha}$ can be real by a good choice of the $\mathrm{Z}_{\alpha}$. Let $\mathrm{Z}_{\alpha}=\mathrm{X}_{\alpha}+i \mathrm{Y}_{\beta}$ and 
$\mathrm{Z}=\mathrm{X}+i \mathrm{Y}$, developing the expression on $\mu(\mathrm{Z})$, we get:

$$
\begin{aligned}
\mu(\mathrm{Z})(\mathrm{P} \uparrow \mathrm{V}) & =\sum_{k=1}^{\infty}\left(\mathrm{X}_{k}^{2}+\mathrm{Y}_{k}^{2}\right) \sum_{\alpha, \beta \in \mathrm{A}}\left(\mathrm{X}_{\alpha, k} \mathrm{Y}_{\beta, k}-\mathrm{Y}_{\alpha, k} \mathrm{X}_{\beta, k}\right)\left(\lambda_{\alpha} d \lambda_{\beta}-\lambda_{\beta} d \lambda_{\alpha}\right) \\
& \text { or } \\
\mu(\mathrm{Z})(\mathrm{P} \uparrow \mathrm{V}) & =\sum_{\alpha, \beta \in \mathrm{A}}\left(\lambda_{\alpha} d \lambda_{\beta}-\lambda_{\beta} d \lambda_{\alpha}\right) \sum_{k=1}^{\infty}\left(\mathrm{X}_{k}^{2}+\mathrm{Y}_{k}^{2}\right)\left(\mathrm{X}_{\alpha, k} \mathrm{Y}_{\beta, k}-\mathrm{Y}_{\alpha, k} \mathrm{X}_{\beta, k}\right),
\end{aligned}
$$

which is a finite sum of summable series.

Now, let us come back to the expression $\mu(\mathrm{Z})=\sum_{k=1}^{\infty}\left|\mathrm{Z}_{k}\right|^{2} \tau_{k}^{*}(\varepsilon)$, it is clear that $\mu(\mathrm{Z})$ is invariant by the diagonal action of $\mathrm{S}^{1}$. And therefore $m([\mathrm{Z}])=\mu(\mathrm{Z})$ is well defined.

25.2. The image under the momentum map of $\mathbf{T}(\mathcal{H})$. The image of the infinite projective space by the momentum map $m$ is the convex hull, in the vector space $\mathcal{T}^{*}$, of the relative momenta

$$
\mathbf{m}_{k}=\operatorname{pr}_{k}^{*}(\varepsilon) \in \mathcal{T}^{*}, k=1 \cdots \infty .
$$

The momentum $\mathbf{m}_{k}$ is indeed the momentum map of the subgroup $\mathrm{S}_{k}^{1}$ of elements of $\mathbf{T}(\mathcal{H})$ for which only the $k$-th component is not the identity.

$$
m(\mathcal{P})=\left\{\sum_{k=1}^{\infty} t_{k} \mathbf{m}_{k} \mid \text { for all } k \in \mathbf{N}^{\star}: t_{k} \geq 0, \text { and } \sum_{k=1}^{\infty} t_{k}=1\right\} .
$$

For any sequence $\left(t_{k}\right)_{k=1}^{\infty}$ such that $\sum_{k=1}^{\infty} t_{k}=1$, and each $t_{k}$ is not negative there exists some $\mathrm{Z} \in \mathcal{H}$ such that $\left|\mathrm{Z}_{k}\right|^{2}=t_{k}$, we can choose $\mathrm{Z}_{k}=\sqrt{t_{k}}$.

\section{References}

[Bou72] N. Bourbaki, Eléments de mathématiques, Espaces vectoriels topologiques, Hermann, Paris, 1972.

[Che77] K. T. Chen, Iterated path integral, Bull. Amer. Math. Soc. 83 (1977) 831-879.

[Don84] P. Donato, Revêtement et groupe fondamental des espaces différentiels homogènes, Thèse de doctorat d'état, Université de Provence, Marseille, 1984.

[DI85] P. Donato and P. Iglesias, Exemple de groupes difféologiques : flots irrationnels sur le tore, Compte Rendu de l'Académie des Sciences 301 (1985).

[HP57] E. Hille and R. Phillips, Functional Analysis and Semigroups, Amer. Math Soc. Colloquium Publ. 31, New York, 1957.

[Igl85] P. Iglesias, Fibrés difféologiques et homotopie, Thèse de doctorat d'état, Université de Provence, Marseille, 1985. http://www.umpa.ens-lyon.fr/ iglesias/articles/ These/PI_These.pdf

[Igl86] P. Iglesias, Difféologie d'espace singulier et petits diviseurs, Compte Rendu de l'Académie des Sciences 302 (1986), 519-522.

[Igl87] P. Iglesias, Connexions et difféologie, in: Aspects dynamiques et topologiques des groupes infinis de transformation de la mécanique, Hermann, Paris, 1987, 61-78.

[Igl95] Patrick Iglesias, La trilogie du Moment, Ann. Inst. Fourier 45 (1995).

[IL90] P. Iglesias and G. Lachaud, Espaces différentiables singuliers et corps de nombres algébriques, Ann. Inst. Fourier (Grenoble) 40 (1990), 723-737. 
[PIZ05] P. Iglesias-Zemmour, Diffeology, eprint, 2005 http://www.umpa.ens-lyon.fr/ iglesias / diffeology/.

[PIZ06] P. Iglesias-Zemmour, Dimension in diffeology, eprint, 2006 http://math.huji.ac.il/ piz/.

[IKZ05] P. Iglesias, Y. Karshon and M. Zadka, Orbifolds as diffeologies, 2005 http://arxiv.org/ abs/math.DG/0501093.

[Kak43] S. Kakutani, Topological properties of the unit sphere of a Hilbert space, Proc. Imp. Acad. Tokyo 19 (1943), 269-271.

[Kui65] N. Kuiper, The homotopy type of the unitary group of Hilbert space, Topology 3 (1965), 19-30.

[McL71] S. McLane, Categories for the Working Mathematician, Springer Verlag, New York, 1971.

[Pal65] R. Palais, On the homotopy type of certain groups of operators, Topology 3 (1965).

[Pal66] R. Palais, Homotopy theory of infinite dimensional manifolds, Topology 5 (1966).

[Sou70] J.-M. Souriau, Structure des systèmes dynamiques, Dunod, Paris, 1970.

[Sou81] J.-M. Souriau, Groupes différentiels, in: Lecture Notes in Mathematics 836, Springer Verlag, New York, 1981, 91-128.

[Sou84] J.-M. Souriau, Groupes différentiels et physique mathématique, in: Collection Travaux en Cours, Hermann, Paris, 1984, 75-79.

[Tyc35] A. Tychonoff, Ein Fixpunktsatz, Math. Ann. 111 (1935), 767-776. 
